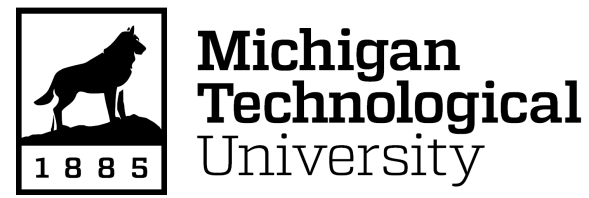

Michigan Technological University Digital Commons @ Michigan Tech

Dissertations, Master's Theses and Master's Reports

2021

Design and Testing of an Open Source Vacuum Oven for Research, Community Recycling, and Additive Manufacturing

Benjamin R. Hubbard

Michigan Technological University, brhubbar@mtu.edu

Copyright 2021 Benjamin R. Hubbard

Recommended Citation

Hubbard, Benjamin R., "Design and Testing of an Open Source Vacuum Oven for Research, Community Recycling, and Additive Manufacturing", Open Access Master's Thesis, Michigan Technological University, 2021.

https://doi.org/10.37099/mtu.dc.etdr/1215

Follow this and additional works at: https://digitalcommons.mtu.edu/etdr

Part of the Manufacturing Commons 


\title{
DESIGN AND TESTING OF AN OPEN SOURCE VACUUM OVEN FOR RESEARCH, COMMUNITY RECYCLING, AND ADDITIVE MANUFACTURING
}

\author{
By \\ Benjamin Robert Hubbard
}

\begin{abstract}
A THESIS
Submitted in partial fulfillment of the requirements for the degree of MASTER OF SCIENCE

In Mechanical Engineering
\end{abstract}

MICHIGAN TECHNOLOGICAL UNIVERSITY

2021

(C) 2021 Benjamin Robert Hubbard 
This thesis has been approved in partial fulfillment of the requirements for the Degree of MASTER OF SCIENCE in Mechanical Engineering.

Department of Mechanical Engineering-Engineering Mechanics

\author{
Thesis Co-Advisor: Joshua M. Pearce \\ Thesis Co-Advisor: $\quad$ Gordon G. Parker \\ Committee Member: Rebecca G. Ong \\ Department Chair: William W. Predebon
}


To my family, my love, and 2Q2. 


\section{Table of Contents}

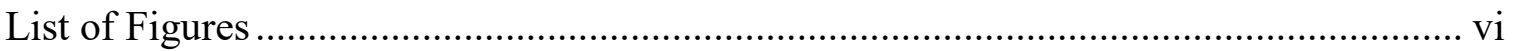

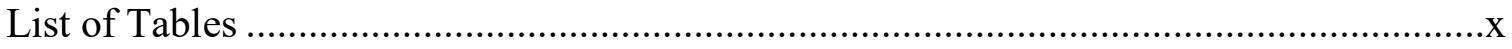

Author Contribution Statement............................................................................... xi

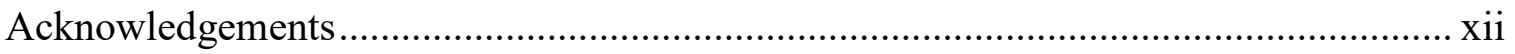

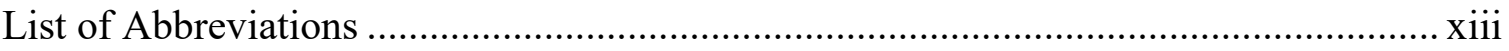

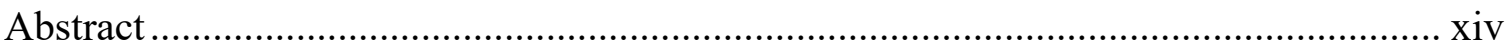

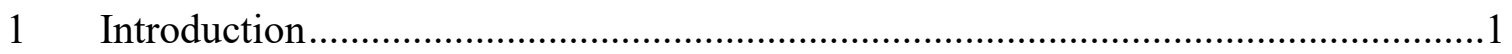

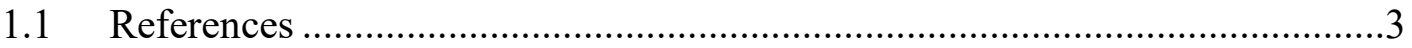

2 Open Source Digitally Replicable Lab-Grade Scales .................................................6

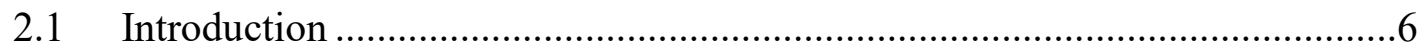

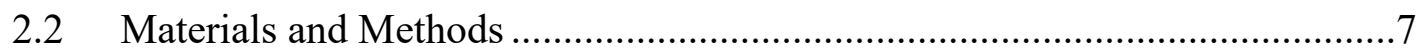

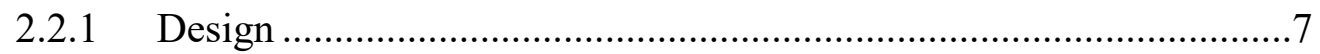

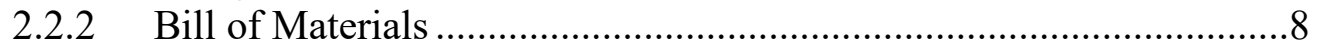

2.2.3 Manufacturing and Assembly .......................................................... 12

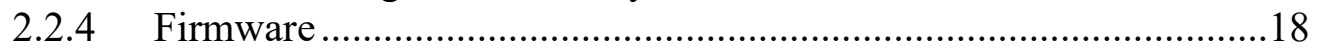

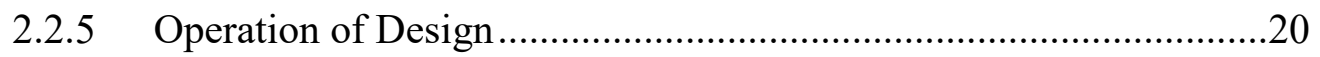

2.2.5.1 Simple Scale............................................................20

2.2.5.2 Lab Scale with Serial Interface ......................................20

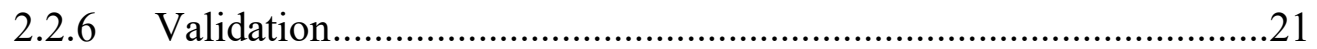

2.2.6.1 Laboratory-Grade Scale Comparison .............................21

2.2.6.2 Self-Calibration of Open Source Scale for $100 \mathrm{~g}$ TAL221 22

2.2.6.3 Measurement on Open Source Scale Using Standard Masses 22

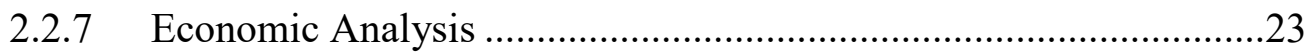

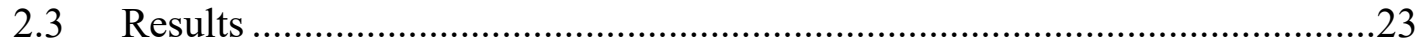

2.3.1 Laboratory-Grade Scale Comparison ...............................................24

2.3.2 Standard Mass Measurements.......................................................27

2.3.3 Economic Analysis ...................................................................28

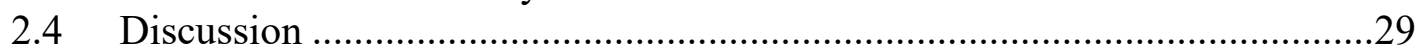

2.4.1 Open Source Scale for Distributed Manufacturing...........................29

2.4.2 Future Technical Work ……………………..................................30

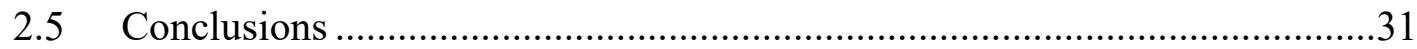

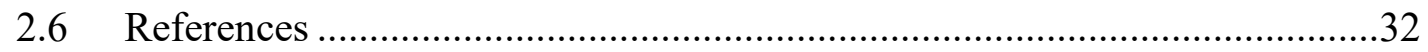

3 Open Source Vacuum Oven for Low-Temperature Drying ……………….............39

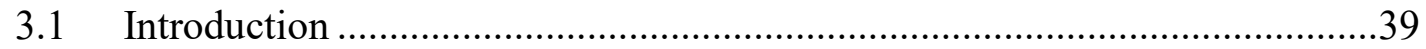




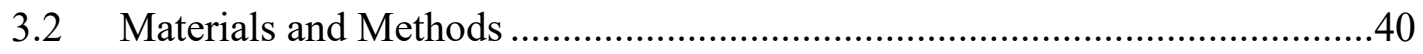

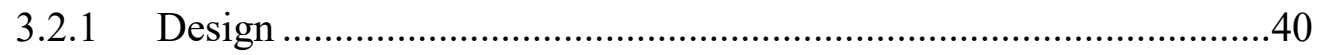

3.2.1.1 Vacuum System ...............................................................

3.2.1.2 Thermal Controls .............................................................41

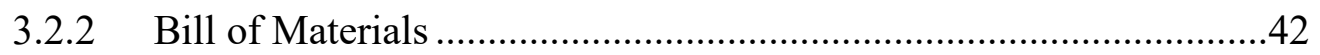

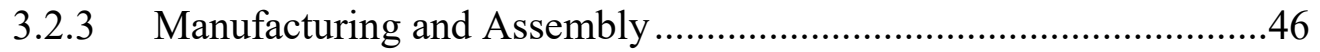

3.2.3.1 Vacuum Chamber .........................................................46

3.2.3.2 Thermal Control System .................................................50

3.2.3.3 Finishing the Assembly...................................................54

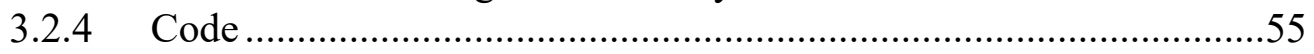

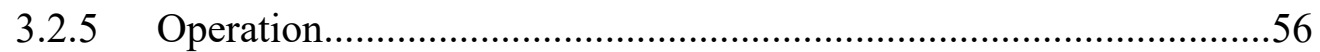

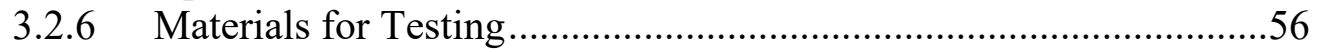

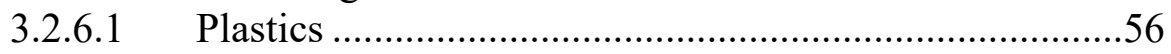

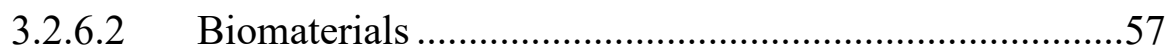

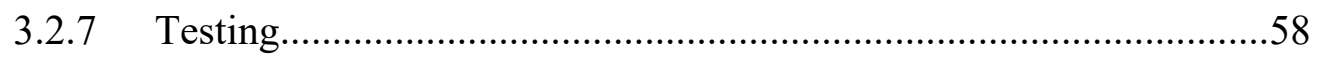

3.2.7.1 Thermistor Calibration..................................................58

3.2.7.2 Temperature Gradient Measurements ..............................58

3.2.7.3 Drying Rate Comparison ................................................59

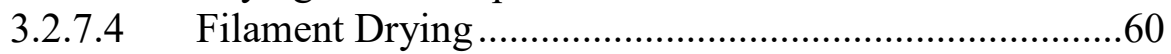

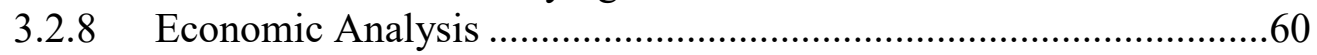

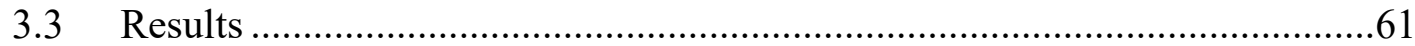

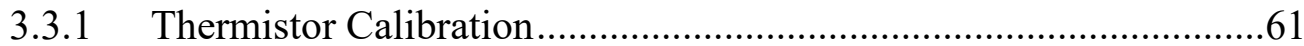

3.3.2 Temperature Gradient Testing ......................................................62

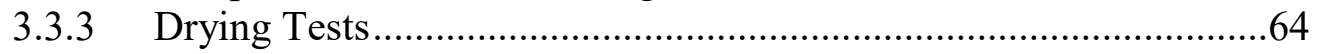

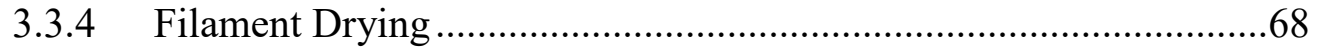

3.3.5 Economic Analysis ...............................................................69

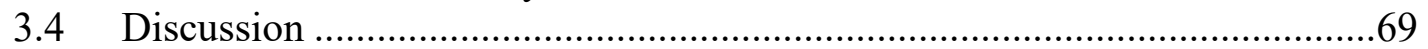

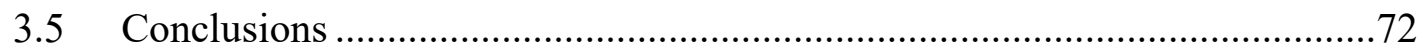

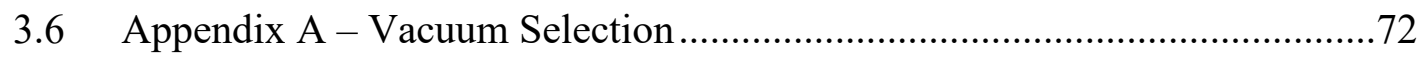

3.7 Appendix B - Wiring Instructions …………….........................................72

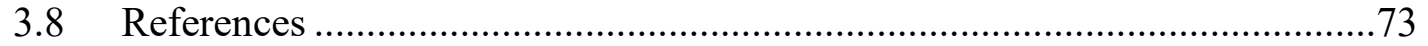

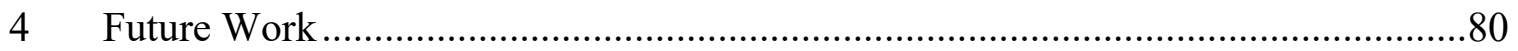

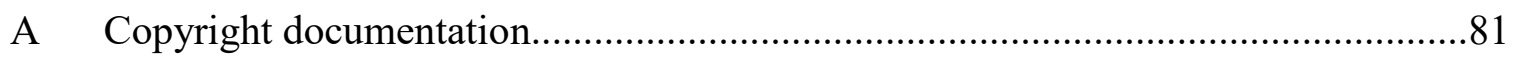




\section{List of Figures}

Figure 2.1. Electrical circuit breadboard layout. Please note that all components connected to and including the LCD are optional.

Figure 2.2. Electrical circuit wiring diagram. Pinouts on the Arduino were selected to reduce the number of required jumper wires when using an Arduino Nano. Pinout selection can be modified in the firmware header file, Pinouts.hpp. Changing micro-controllers or pinouts may require different circuit parts than those listed in

Table 2.1.

Figure 2.3. The circuit was assembled on a solder circuit board using jumper wires and header pins. The female header pins connect to the Arduino Nano and the HX711. The male header pins connect to the load cell and the LCD. The tare push-button is attached via twisted pair to allow installation on the face of the scale. (a) The soldered components were arranged in such a way to minimize wire use and to properly place the Arduino when installed in the base. (b) Shows the final circuit board.

Figure 2.4. Key features of the base include standoffs to mount the load cell, locators for the screen and circuit board, and a slot for access to the Arduino Nano's USB port. The base also has a snap-seam to attach the cover to the base, enclosing the internal components.

Figure 2.5. The circuit board fits into the bosses on the right side of the base to hold it in place. The twisted pair was fed through the hole for the push-button and a set of female-male jumper wires were connected to the load cell header pins to reach the TAL221 wires.

Figure 2.6. The LCD is held in place by two locators behind the slot for the screen. Its wires are tucked under a plastic wire manager attached to the TAL220 boss, then connected to the circuit board.

Figure 2.7. (a) The TAL220 loadcell should be installed with the wires leading off the end of the loadcell attached to the base - the M5 tapped holes. Please note that the button is also installed. (b) After snapping on the lid, the bed can be attached to the free end of the load cell using two M4 bolts.

Figure 2.8. (a) The cover must be sandwiched between the loadcell and the bed prior to attaching to the base. Note the direction the loadcell is aimed so it will rest on the TAL221 boss when the cover is snapped on. (b) After connecting the loadcell to the controller, the cover is snapped on and the TAL221 is screwed to the base using two M3 bolts. This must be done by feel, but does not take very long........17

Figure 2.9. The scale returns a readout similar to the one shown during initialization. The readout indicates the action in progress, success, and returns the sensitivity value when reading from memory.

Figure 2.10. The $5 \mathrm{~kg}$ loadcell results are shown (a) The standard deviation (sample of 5 measurements) averaged to $16.3 \mathrm{mg}$ for the open source scale. This is large relative to the APX402 and the A-160. The A-160 performed at a very small 
standard deviation relative to the other scales, causing its bars to be difficult to see at the bottom of the chart; (b) The absolute value of the difference between each scale and the A-160 is shown. The open source scale averaged a difference of $36.3 \mathrm{mg}$.

Figure 2.11. The $500 \mathrm{~g}$ loadcell test results are shown. (a) The standard deviation (sample of 5 measurements) averaged to $20.7 \mathrm{mg}$ for the open source scale. This is large relative to the lab-grade scales; (b) The absolute value of the difference between the average measurements by the A-160 and the other two scales are shown. The open source scale average measurement was within $14.2 \mathrm{mg}$ of the A-160, on average.

Figure 2.12. The $100 \mathrm{~g}$ loadcell test results are shown. (a) The standard deviation (sample of 5 measurements) averaged to $5.0 \mathrm{mg}$ for the open source scale. This is close to that of the lab-grade scales (on the same order as the APX402); (b) The absolute value of the difference between the average measurements by the $500 \mathrm{~g}$ TAL221 and the $100 \mathrm{~g}$ TAL221 are shown. The $100 \mathrm{~g}$ TAL221 average measurement was within $19.8 \mathrm{mg}$ of the $500 \mathrm{~g}$ TAL221, on average.

Figure 2.13. The standard mass test results are shown. (a) The standard deviation (sample of at least 48 measurements) increased with the range of the load cell; there is little difference between the PLA housing and wood frame. (b) The absolute value of the difference between the average measurements of each scale and the nominal mass of the weights is shown. The accuracy range for the weights is indicated by a red line.

Figure 3.1. Insulation was cut to fit the specific vacuum chamber that was purchased. (a) Circles of insulation to cover the base and lid; (b) A strip of insulation to be wrapped around the circumference of the chamber to build an air gap.

Figure 3.2. Air ejector connector cross-sections, highlighting internal geometry where air flows. (a) Intake connector, where high pressure air enters the ejector; (b) Vacuum connector, where evacuated air flows from the chamber to the ejector and vacuum pressure is measured.

Figure 3.3. Assemble the air ejector. (a) Thread the 3-D printed connectors into the air ejector, using PTFE tape if necessary; (b) Attach the vacuum gauge to the hose barb.

Figure 3.4. Process to connect the air ejector to the lid. Note that the photographed part is grey here, where it was orange in prior photos. (a) Feeding the ejector through the insulation, then an M10 washer (nylon or rubber); (b) Feeding the ejector through the lid, then another washer, then secure with an M10 nut.

Figure 3.5. If installed, the heater power cable and sensor wires may be fed up the wall of the chamber for strain relief, but this is not required.

Figure 3.6. Install insulation on the chamber wall. (a) First, attach strips of insulation to build an air gap and span the protruding components on the chamber walls; (b) Next, install the large piece of insulation, fully covering the walls of the chamber. 
Figure 3.7. The circuit is a voltage divider and a pair of signal wires to control the relay.

Figure 3.8. The completed circuit is wired to connect to a relay and measure a single thermistor.

Figure 3.9. Assembly of the electronics for the thermal controller. (a) The Arduino installed on the circuit; (b) The signal wires attached to the relay.

Figure 3.10. The heating element was adhered to the base of the chamber, with the thermistor secured on the interface.

Figure 3.11. The power cable was prepared for connection to the relay. (a) First, it was cut in half. Only one wire needed to be cut, but both were cut in this case; (b) Next, one pair of wires was immediately re-joined with a wire nut. .....................54

Figure 3.12. The power cable was secured into the load terminals of the relay................54

Figure 3.13. The fully assembled vacuum oven. .55

Figure 3.14. The test setup for measuring temperature gradient. (a) Three thermistors were mounted on a 3-D printed jig, mounting them 0,25 , and $50 \mathrm{~mm}$ above the base of the chamber; (b) For the second test, a heat sink was added in the mix of rPET to aid heat transfer throughout the material.

Figure 3.15. The full Steinhart-Hart equation yields this temperature curve, showing the thermistor's sensitivity decreasing as temperature increases.

Figure 3.16. The calculated Beta values have varying accuracy as a function of resistance. These curves show that Beta of 3920 or 3950 maintain error within $1{ }^{\circ} \mathrm{C}$ for the majority of the temperature range under assessment.

Figure 3.17. Temperature gradient measurements. (a) Chamber loosely packed with rPET; (b) Chamber loosely packed with rPET, plus a metal cylinder, referred to as a heat sink.

Figure 3.18. Temperature gradient measurements with the inside surface temperature used for control. (a) Chamber empty and open to the air, average heater temperature $79{ }^{\circ} \mathrm{C}$ at steady state; (b) Chamber loosely packed with $\mathrm{rPET}$, average heater temperature $68{ }^{\circ} \mathrm{C}$ at steady state.

Figure 3.19. Dehydration measurements on small (10 g) samples of rPET. Increasing the oven temperature from 70 (left) to $80 \circ \mathrm{C}$ (right) caused a $40 \%$ decrease in drying time. (a) Sample percent initial mass with the vacuum oven set to $70{ }^{\circ} \mathrm{C}$. The vacuum oven was $25 \%$ slower than the dehydrator at this setting; (b) Sample percent initial mass with the vacuum oven set to $80{ }^{\circ} \mathrm{C}$ and the dehydrator still set to $70{ }^{\circ} \mathrm{C}$. In this case, the vacuum oven was $40 \%$ faster than the dehydrator. (c) The energy consumed during the $70 \circ \mathrm{C}$ test. The dashed lines are summed in the blue aggregate energy for the oven. (d) The energy consumed during the $80{ }^{\circ} \mathrm{C}$ test.

Figure 3.20. Dehydration of large $(350 \mathrm{~g})$ samples of rPET. This test was cut short because it clearly indicated that the vacuum oven could not manage the large 
mass of plastic and water. (a) The percentage of initial mass as a function of time. (b) The energy consumption of each device. The energy consumption of the vacuum is shown as an aggregate of the two components, the heater and the vacuum

Figure 3.21. $500 \mathrm{mg}$ samples of the consortium were dehydrated in a petri dish. (a) The wet culture, prior to drying; (b) The fully dehydrated culture.

Figure 3.22. Dehydration measurements on consortium of microorganisms. (a) Percent initial mass for each sample; (b) energy consumption. The dashed oven vacuum and oven heater curves were summed to form the aggregate oven curve.

Figure 3.23. The dried filament showed significantly stronger layer adhesion and smoother surface finish.

Figure 3.24. A $1 \mathrm{~kg}$ spool of PLA filament was dried in the vacuum oven. The change in mass was around $0.4 \mathrm{~g}$, compared to the initial mass of $1245.7 \mathrm{~g}$.

Figure 3.25. Illustration of wire solder labels. The top row is row 10, and it contained a row of header pins. A wire needed to connect to the header pin at L10. To do this, the wire was soldered into L9, then bent over onto L10 (which was already occupied by a header pin) and soldered in place. This wire would be labeled 'L9 : L10 


\section{List of Tables}

Table 2.1. Visual bill of materials separated by mechanical and electrical components.....8

Table 2.2. Print settings for the 3-D printed components. .13

Table 2.3. The average standard deviation for each loadcell during repeatability testing is compared as a percentage of the loadcell's total range. These results suggest that the precision of the loadcell is a function of its range and style.

Table 2.4. The average standard deviation for each loadcell during testing with standard masses is compared as a percentage of the loadcell's total range. These results reinforce the idea that the precision of the loadcell is a function of its range and style.

Table 2.5. The cost of the scale varies based on configuration and initial investment. Using a wall outlet for power (as opposed to a computer) costs an extra $\$ 4.00 \ldots .29$

Table 2.6. Several proprietary scales listed online were compared in price and capability to the open source scale. The comparison indicates the cost savings allowed by the open source scale, which vary greatly depending on the functionality and

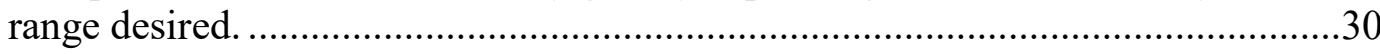

Table 3.1. Visual bill of materials separated between the two primary components. .......43

Table 3.2. Print Settings for 3-D Printed Parts (PLA) ........................................................48

Table 3.3. Optimized reference resistances for several operating temperature ranges......51

Table 3.4. Thermistor calibration measurements............................................................58

Table 3.5. Steinhart-Hart full model calibration..............................................................61

Table 3.6. Steinhart-Hart simplified model calibration. ....................................................62

Table 3.7. Summary of the dehydration test results.........................................................68

Table 3.8. Cost breakdown of the vacuum oven..............................................................69

Table 3.9. Cost comparison of the open source vacuum oven with other devices on the market. 


\section{Author Contribution Statement}

Chapters 2 and 3 are published, co-authored works or are intended for submission as such.

Chapter 2 was authored by Benjamin R. Hubbard and Joshua M. Pearce.

Conceptualization, B.R.H. and J.M.P.; methodology, B.R.H. and J.M.P.; software, B.R.H.; validation, B.R.H.; formal analysis, B.R.H. and J.M.P.; investigation, B.R.H. and J.M.P.; resources, J.M.P.; data curation, B.R.H.; writing — original draft preparation, B.R.H. and J.M.P.; writing - review and editing, B.R.H. and J.M.P.; visualization, B.R.H.; funding acquisition, J.M.P.

Chapter 3 was authored by Benjamin R. Hubbard, Lindsay I. Putman, Stephen M. Techtmann, and Joshua M. Pearce.

Conceptualization, B.R.H. and J.M.P.; methodology, B.R.H., L.I.P., S.M.T., J.M.P.; software, B.R.H.; validation, B.R.H.; formal analysis, B.R.H. and J.M.P.; investigation, B.R.H. and J.M.P.; resources, J.M.P.; data curation, B.R.H.; writing — original draft preparation, B.R.H., L.I.P., S.M.T., and J.M.P.; writing-review and editing, B.R.H., L.I.P., S.M.T., and J.M.P.; visualization, B.R.H.; funding acquisition, S.M.T. J.M.P. 


\section{Acknowledgements}

Thank you, Dr. Pearce, for driving my work and encouraging me to continue chasing discovery. Thanks to all of the faculty at MTU for making my education such a challenging and engaging experience.

Thank you, Mom, Dad, and Summer, for cheering me on. Thanks to my college family for keeping me company and helping me learn.

Thank you, my friends in industry, who have taught me so much about being an engineer and helped guide me along the journey I have taken.

Thank you, open source community, for driving the world toward a society in which information is a treasure so valuable that we cannot help but share it. 


\section{List of Abbreviations}

DRAM - Digital Recycling and Additive Manufacturing

FOSS - Free and Open Source Software

FOSH - Free and Open Source Hardware

OS - Open Source

rPET - Recycled Polyethylene Terephthalate 


\section{Abstract}

To aid the continuing growth of open source tools in the research community, this thesis presents two broadly useful instruments which can be built and operated for a fraction of the cost of equivalent or inferior commercial products. The first device is a digitallyreplicable scale, which provides a single framework for making measurements on the order of milligrams all the way up to tens of kilograms by supporting multiple types of load cells. The second device is a vacuum oven, which is validated using the first. The open source vacuum oven facilitates the dehydration of materials at low temperature, providing a fast method for drying thermally sensitive materials such as post-consumer waste plastics for recycling and biological specimens. These tools are provided with publicly available designs of both hardware and software components, assembly instructions, and performance data. This provides researchers with the opportunity to validate and improve upon the existing tools, customizing them to meet specific needs in the lab. 


\section{Introduction}

The open source community, since its conception over two decades ago, has been reaching into an increasingly broad variety of hardware and software topics. The open source community started in the software industry. Free and Open Source Software (FOSS) is computer software that is available in source code form and can be used, studied, copied, modified, and redistributed either without restriction or with restrictions only to ensure that further recipients have the same rights under which it was obtained: free, or libre [1-5]. It is clear that there are benefits of FOSS over proprietary and restrictive approaches to technical development [1-8]. The power of the libre approach is that large-scale collaboration on technical problems results in superior design and innovative solutions with lower associated costs due to continuous improvement $[1,9]$. There is widespread agreement that FOSS is more reliable and relevant to users [10] (in no small part due to the fact that FOSS users are often co-developers [11]). FOSS is so successful that it has become prominent enough in the software industry to change the career trajectory of software developers [12]. In addition FOSS now dominates major areas of computing including: 1) Android, an open source-based operating system, is the world's most popular operating system [13], 2) all supercomputers (including Michigan Tech's own super computer: Superior) [14], 3) 90\% of cloud servers, $82 \%$ of smartphones and $62 \%$ of embedded systems run on open-source operating systems [15], 4) more than $70 \%$ of "internet of things" devices also use open-source software [16] and 5) $90 \%$ of the Fortune Global 500 pay for the open-source Linux operating system from Red Hat [17], a company that was recently acquired by IBM and makes billions of dollars a year [18] for the service they provide on top of the product that can be downloaded for free. The Linux operating system extends beyond the corporate world to high-budget areas of research, including the International Space Station and the Large Hadron Collider [19]. Major internet-based corporations use and develop FOSS at an astounding rate including: Amazon, Alphabet (Google), Twitter and Facebook. These companies and others use FOSS because of superior technical performance, more flexible design and reduced research and development $(\mathrm{R} \& \mathrm{D})$ costs $[20]$.

Although, years ago, even FOSS proponents believed that the open source way could not be removed from the digital realm because of the necessity of building physical objects [21], these views changed with the introduction of low-cost digital distributed manufacturing tools [22]. The most prominent of such open source tools is the selfreplicating rapid prototyper (RepRap) 3-D printer community [23-26]. The RepRap project itself was only possible because of another open hardware project - the Arduino microcontroller. Arduino is an open hardware/software platform that powers thousands of projects around the world, including the two presented in this thesis. Following the introduction of distributed automation and manufacturing tools, free and open source hardware (FOSH) has become a force in the technical community [27]. FOSH is hardware whose design is made publicly available so that anyone can study, modify, manufacture, distribute, and sell the design or pieces of hardware based on that design [28]. Free and open source-based technical development provides a strong list of competitive advantages including: 1) more participants than proprietary innovation within firms [1-8] (e.g. thousands of developers have worked on Linux and the RepRap 
projects), 2) it is less encumbered by intellectual property issues [29-31] (e.g. no time is wasted with lawyers negotiating license agreements, non-disclosure agreements (NDAs), or ensuring that an invention does not infringe on patent claims), and 3) innovation occurs at steeper rates [32]. The scientific community in particular has made a rapid shift to adopt libre hardware as it reduces costs by $90-99 \%$, while improving control and allowing customization [33-35]. In addition to publishing free and open source technology in traditional publishing, new academic journals are flourishing such as HardwareX (publishing libre hardware) and the Journal of Open Hardware, which covered the annual Gathering for Open Science Hardware [36] and libre hardware-based business models [37]. Some libre hardware designs have been shown to be growing at an exponential rate [33]. In addition, the academic community is rapidly embracing libre for both FOSS and FOSH, with the latter lagging by about 20 years based on indexing in Google Scholar [38].

FOSH has particularly strong involvement in environmental efforts to reduce waste through distributed recycling and additive manufacturing (DRAM). The broad distribution, availability, and hackability of 3-D printers has built a framework for individuals, makerspaces, and research labs to create closed-loop recycling within their own spaces. Recyclebots, devices which convert plastic waste into 3-D printing filament with diametral tolerances of $+/-0.05 \mathrm{~mm}$ [39], whether from old 3-D prints or consumer products such as food containers, have been developed and made publicly available [40]. Direct-extrusion 3-D printers exist that use waste plastic in its shredded form allow the filament-spooling process to be skipped, reducing the amount of hardware required to return waste plastic back to a useful form [41]. Plastic has a limit on the number of cycles through which it can be recycled by mechanical breakdown and remelting, after which its chemical structure breaks down to the point of degrading mechanical properties. The plastics industry manages this by mixing a small portion of recycled material with virgin material in order to ensure mechanical strength. Ongoing research is exploring manners of characterizing waste plastic in order to quantify its viability for reuse, which will improve the efficiency of recycling. An existing limitation on print quality for virgin and recycled plastics in DRAM is that product quality is limited by the moisture content of the material during processing. High volumes of water in the chemical structure of (or on the surface of) plastics cause chemical breakdown of some of the polymer at high temperatures. Existing methods for drying plastics on a small scale are limited to hot air dryers and passive desiccant solutions, which address the issue of surface water, but do not have a significant effect on water absorbed into polymer chains.

This thesis seeks to close this gap in available drying hardware by presenting the designs and data for an open source vacuum oven. Vacuum drying offers many advantages over hot-air and both passive and active desiccant drying, including higher rates of drying, improved energy efficiency, and lower required temperatures for drying, which allows the dehydration of heat-sensitive materials. Current closed source vacuum ovens available cost upwards of $\$ 2000$. The development and validation of this dryer called for an effective manner for tracking mass over time. Existing comprehensive solutions which offer precision and a serial interface for logging data are closed source and relatively expensive [42]. These products are also limited to a single range and precision for 
measuring mass. Both of these instruments, the digital scale and the vacuum oven, offer high value to the research community as a whole, and as such are presented in a broad context, given their extensive applications beyond DRAM.

Chapter 2 presents an open source digital scale framework which can be produced for around $\$ 30$ and is easily adapted to varying requirements including the range, type, and precision of load cell used to complete measurements. Testing on the scale showed that it can achieve measurement precision as fine as $+/-5 \mathrm{mg}$, depending on the load cell in use. The load cell styles supported by this design range from $100 \mathrm{mg}$ up to $100 \mathrm{~kg}$. The highest capacity load cell documented was a $5 \mathrm{~kg}$ load cell, which provided measurement precision of $+/-40 \mathrm{mg}$. The scale can be used for a variety of applications, including chemical experiments, load sensing applications, active application and measurement of force, long term mass-tracking measurements, and more. The scale itself is not limited to use in the 3-D printed body, which was designed for it, rather can be used in any environment where a load cell can be installed.

Chapter 3 presents an open source vacuum oven, which can be reproduced for around \$360. The oven was shown to be effective for drying flakes of recycled PET (e.g. water bottle plastic) as well as a consortium of bacteria, which is being developed to recycle waste PET into food rather than a 3-D printing feedstock. The vacuum oven was validated by running dehydration tests on multiple materials and measuring the change in mass over time using the digital scale described in Chapter 2. The vacuum oven spans the gap between simple hot-air dryers and much more expensive commercial vacuum dryers. It is directly applicable to dehydrating material to be used in DRAM, as well as for use in other processes requiring low drying temperatures including bio-material preservation, and chemical processing.

The development of these instruments exposed several avenues along which further research could be explored. Chapter 4 discusses these areas of future work in the interest of sparking continued work in the FOSH community.

\subsection{References}

1. Raymond, E. The cathedral and the bazaar. Knowl. Technol. Policy 1999, 12, 23-49, doi:10.1007/s12130-999-1026-0.

2. Lerner, J.; Tirole, J. Some Simple Economics of Open Source. J. Ind. Econ. 2002, 50, 197-234, doi:10.1111/1467-6451.00174.

3. Bonaccorsi, A.; Rossi, C. Why Open Source software can succeed. Res. Policy 2003, 32, 1243-1258, doi:10.1016/S0048-7333(03)00051-9.

4. Lakhani, K.R.; Von Hippel, E. How open source software works: "Free" user-to-user assistance. Res. Policy 2003, 6, 923-943, doi:10.1016/S0048-7333(02)00095-1.

5. Lakhani, K.; Wolf, R.J. Why Hackers Do What They Do: Understanding Motivation and Effort in Free/Open Source Software Projects. 2003, doi:10.2139/ssrn.443040.

6. Vetter, G.R. Commercial Free and Open Source Software: Knowledge Production, Hybrid Appropriability, and Patents. Fordham Law Rev. 2008, 77, 2087. 
7. DiBona, C.; Ockman, S. Open Sources: Voices from the Open Source Revolution; O’Reilly Media, Inc.: Sebastopol, CA, USA, 1999; ISBN 978-0-596-55390-6.

8. Deek, F.P.; McHugh, J.A.M. Open Source: Technology and Policy; Cambridge University Press: Cambridge, UK, 2007; ISBN 978-1-139-46873-2.

9. Söderberg, J. Hacking Capitalism: The Free and Open Source Software Movement; Routledge: Abingdon, UK, 2015; ISBN 978-1-135-91639-8.

10. Kogut, B.; Metiu, A. Open source software development and distributed innovation. Oxf. Rev. Econ. Policy 2001, 17, 248-264, doi:10.1093/oxrep/17.2.248.

11. von Hippel, E. Innovation by User Communities: Learning from Open-Source Software. MIT Sloan Manag. Rev. Camb. 2001, 42, 82-86.

12. Riehle, D. How Open Source Is Changing the Software Developer's Career. Computer 2015, 48, 51-57, doi:10.1109/MC.2015.132.

13. Smith, M. Android Now the World's Most Popular Operating System. CSOnline, 2017. Available online: https:/www.csoonline.com/article/3187011/mobilewireless/android-is-now-the-worlds-most-popular-operating-system.html (accessed on 3 May 2018).

14. Vaughan-Nichols, S.J. Linux Dominates Supercomputers as Never Before |ZDNet. Available online: https://www.zdnet.com/article/linux-dominates-supercomputers-asnever-before/ (accessed on 3 May 2018).

15. Sean Michael Kerner. Linux Foundation on Track for Best Year Ever as Open Source Dominates. ServerWatch 2018, 1.

16. Christine Hall. Survey Shows Linux the Top Operating System for Internet of Things Devices. IT Pro 2018.

17. Red Hat Enterprise Linux Operating System. Available online: https://www.redhat.com/en/technologies/linux-platforms/enterprise-linux (accessed on 1 April 2021).

18. Red Hat Reports Fourth Quarter and Fiscal Year 2018 Results. Available online: https://www.redhat.com/en/about/press-releases/red-hat-reports-fourth-quarter-andfiscal-year-2018-results (accessed on 1 April 2021).

19. Dan Price. 9 Ways Linux Is Taking Over the World. MUO 2019.

20. Mockus, A.; Fielding, R.T.; Herbsleb, J.D. Two Case Studies of Open Source Software Development: Apache and Mozilla. ACM T. Softw. Eng. Methodol. 2002, 11, 309-346, doi:10.1145/567793.567795

21. Stallman, R. M. Richard Stallman -- On "Free Hardware". LinuxToday.com, 1999. Available online: http://www.linuxtoday.com/news story.php3?ltsn=1999-06-22005-05-NW-LF (accessed on May 3, 2018).

22. Stallman, R.M. Free Hardware and Free Hardware Designs. Wired. GNU.org. Available online: http:/www.gnu.org/philosophy/free-hardware-designs.en.html (accessed on May 3, 2018).

23. Sells, E.; Bailard, S.; Smith, Z.; Bowyer, A.; Olliver, V. RepRap: The Replicating Rapid Prototyper-Maximizing Customizability by Breeding the Means of Production. Proceedings in the World Conference on Mass Customization and Personalization, Cambridge, MA, USA. 2010.

24. Jones, R.; Haufe, P.; Sells, E.; Iravani, P.; Olliver, V.; Palmer, C.; Bowyer, A. RepRap-the Replicating Rapid Prototyper. Robotica 2011, 29 (01): 177-91. 
25. Bowyer, A. 3D Printing and Humanity's First Imperfect Replicator. 3D Printing and Additive Manufacturing 2014, 1 (1): 4-5.

26. Anzalone, G.C.; Wijnen, B.; Pearce, J.M. Multi-material additive and subtractive prosumer digital fabrication with a free and open-source convertible delta RepRap 3D printer. Rapid Prototyping Journal, 2015, 21(5), 506-519, doi: 10.1108/RPJ-092014-0113

27. Gibb, A.; Abadie, S. Building open source hardware: DIY manufacturing for hackers and makers, 1st ed; Addison-Wesley Professional: Boston, MA, USA, 2014.

28. Open Source Hardware Association. Definition (English) - Open Source Hardware Association 2018. Available online: https://www.oshwa.org/definition// (accessed on May 4, 2018).

29. Chesbrough, H. W. Open Innovation: The New Imperative for Creating and Profiting from Technology; Harvard Business Press, 2006; ISBN 978-1-4221-0283-1.

30. Huizingh, E. K. R. E. Open innovation: State of the art and future perspectives. Technovation 2011, 31, 2-9, doi:10.1016/j.technovation.2010.10.002.

31. Yu, D.; Hang, C. C. Creating technology candidates for disruptive innovation: Generally applicable R\&D strategies. Technovation 2011, 31, 401-410, doi:10.1016/j.technovation.2011.02.006.

32. Foss, N. J.; Pedersen, T. Organizing knowledge processes in the multinational corporation: an introduction. J Int Bus Stud 2004, 35, 340-349, doi:10.1057/palgrave.jibs.8400102.

33. Pearce, J. M. Building Research Equipment with Free, Open-Source Hardware. Science 2012, 337 (6100): 1303-1304. DOI: 10.1126/science. 1228183

34. Pearce, J. Open-Source Lab: How to Build Your Own Hardware and Reduce Research Costs. 1st ed. Elsevier: Waltham, MA, USA, 2014.

35. Baden, T.; Chagas, A.; Marzullo, T.; Prieto-Godino, L.; Euler, T. Open Laware: 3-D Printing Your Own Lab Equipment. PLoS Biology 2015, 13 (3).

36. Dosemagen, S., Liboiron, M. \& Molloy, J., (2017). Gathering for Open Science Hardware. Journal of Open Hardware 2016. 1(1), p.4. 10.5334/joh.5

37. Pearce, J. M. Emerging Business Models for Open Source Hardware. Journal of Open Hardware 2017, 1, doi:10.5334/joh.4.

38. Pearce, J.M., 2018. Sponsored libre research agreements to create free and open source software and hardware. Inventions, 3(3), p.44.

39. Petsiuk, A.L.; Pearce, J.M. Open Source 3-D Filament Diameter Sensor for Recycling, Winding and Additive Manufacturing Machines. 2020.

40. Woern, A.L., McCaslin, J.R., Pringle, A.M. and Pearce, J.M., 2018. RepRapable Recyclebot: Open source 3-D printable extruder for converting plastic to 3-D printing filament. HardwareX, 4, p.e00026.

41. Woern, A.L., Byard, D.J., Oakley, R.B., Fiedler, M.J., Snabes, S.L. and Pearce, J.M., 2018. Fused particle fabrication 3-D printing: Recycled materials' optimization and mechanical properties. Materials, 11(8), p.1413.

42. Hubbard, B.R.; Pearce, J.M. Open-Source Digitally Replicable Lab-Grade Scales. Instruments 2020, 4, 18. https://doi.org/10.3390/instruments4030018 


\section{Open Source Digitally Replicable Lab-Grade Scales}

This study provides designs for a low-cost, easily replicable open source lab-grade digital scale that can be used as a precision balance. The design is such that it can be manufactured for use in most labs throughout the world with open source RepRap-class material extrusion-based 3-D printers for the mechanical components and readily available open source electronics including the Arduino Nano. Several versions of the design were fabricated and tested for precision and accuracy for a range of load cells. The results showed the open source scale was found to be repeatable within $50 \mathrm{mg}$ with multiple load cells, with even better precision $(5 \mathrm{mg})$ depending on load cell range and style. The scale tracks linearly with proprietary lab-grade scales, meeting the performance specified in the load cell data sheets, indicating that it is accurate across the range of the load cell installed. The smallest load cell tested (100 g TAL221) offers precision on the order of a commercial digital mass balance. The scale can be produced at significant cost savings compared to scales of comparable range and precision when serial capability is present. The cost savings increase significantly as the range of the scale increases and are particularly well-suited for resource-constrained medical and scientific facilities.

\subsection{Introduction}

The incredible success of free and open source development of software [1,2] is being rapidly adopted by the hardware community $[3,4]$ as it enables scientific equipment designers to rapidly build upon one another's work [5-7]. This has resulted in a democratization of design building on open source designs [8-10] often with open source tools that enable a true digital distributed manufacturing [11-14]. The most robust example is the self-replicating rapid prototype (RepRap) fused filament fabrication (FFF)-based 3-D printer [15-17] that has brought the cost of both rapid prototyping and additive manufacturing low enough to be used by the masses [18-21]. FFF-based 3-D printers derived from RepRaps now have 3-D printed parts with sufficient mechanical strength [22] to be used for final functional parts even if printed on machines that only cost a few hundred dollars. This has resulted in an explosion of open source digitally fabricated instruments and a repository of designs housed at the $\mathrm{NIH}[5,6,23,24]$. There are many examples of progressively more sophisticated open source 3-D printed parts being used to build chemical mixing systems [22-25], mechanical components for optics setups [26-31] and microscopes [32-34], instruments to test water quality testing [3538], various types of syringe pumps [39-43] that are combined with other components to make complete systems for making microfluidics and metafluidics [44-47]. Although the most important feature of open source 3-D printable instruments is the ease with which scientists can customize a tool, in general, they are also much less expensive than equivalent (and often technically inferior) commercial proprietary systems [6,23,24,48$50]$ and provide a high return on investment [51,52]. This advantage expands as the device is made with a higher percentage of digitally replicable components [53]. To continue this tradition of standing on the shoulders of open hardware giants [54], this paper describes the design of an open source largely 3-D printed digital scale. A digital scale is a scientific instrument that provides fast measurements of mass generally with an 
accuracy range of $100 \mathrm{mg}$ to $10 \mathrm{mg}$ and have easy-to-read automatic liquid crystal displays. (Accuracy refers to closeness of the measurements to a known value of the mass. On the other hand, precision refers to the closeness of the measurements to one another, which is commonly stated as a single standard deviation of several tests and is independent of the accuracy (i.e., statistical dispersion).) Digital scales are used in a wide range of scientific applications including chemical research, genomics, drug discovery, and proteomics. Scales are classified by precision; where a precision balance has a measurement resolution of $1 \mathrm{mg}$, an analytical balance has one of $100 \mu \mathrm{g}$ and a micro balance has one of $100 \mathrm{ng}$. On the high-end of scales, an open source quartz crystal microbalance (OpenQCM) [55] uses an open source Arduino Nano and is already well established in the scientific literature [56-60].

Thus, this study provides the designs for a low-cost, easily replicable, open source labgrade digital scale that can be used as a precision balance depending on configuration. The design is such that it can be manufactured for use in most labs throughout the world with open source RepRap-class material extrusion-based 3-D printers for the mechanical components and readily available open source electronics including the Arduino Nano. In addition, a validation procedure for quantifying the accuracy and precision is provided. Several versions of the design were fabricated and tested for a range of load cells, and the results are discussed in the context of resource-constrained medical and scientific facilities.

\subsection{Materials and Methods}

\subsubsection{Design}

This series of open source scales is based on load cells, which are devices designed to measure weight or force. The load cells used in this study are strain gage load cells, which convert strain (i.e., change in length) of a material into an electrical signal proportional to the force applied [61]. The load cell in built to provide a linear output that is, a given load applied to the load cell results in a given change in the measured value (regardless of the absolute load applied) [61]. This allows the load cell to be calibrated using a single non-zero data point. This series of scales was designed to be easily manufacturable, require minimal components, and offer the functionality necessary for a basic digital scale, a precision balance, and an analytical balance. Component location is accomplished entirely by features on the 3-D printed components including bosses and snap-fit joints, meaning that the only fasteners required are those that hold the loadcell in place. The assembly was designed to fully enclose all electronics, limiting airflow, which has been observed to affect the output of the loadcell amplifier.

At the time of writing, the scale is designed to accommodate two sizes of single-point parallel beam loadcells - TAL220 and TAL221 - but is set up to enable fast adaptation to other models. These were selected first due to their low cost, wide selection of weight ranges, and insensitivity to moment loading on the bed, which aids in measurement repeatability [61]. The base and bed were designed to be easily modified to accommodate other loadcell styles as required. 
The open source scale systems were designed for use in two settings: (1) as an independent digital scale with a displayed output which can be tared (the reference for a reading of zero can be offset to ignore a known mass, such as that of a container) and calibrated (the output can be corrected to present the true mass by loading the scale with a known mass) without use of a computer, and (2) as a serially connected logging instrument for a laboratory setting and data-logging. The serial capability also allows this scale to be constructed without an LCD, offering a significant cost reduction for uses that do not require a display, such as automated weighing or cases where a computer will always be used as the power supply.

To accomplish independent functionality, components were specified to ensure that the entire scale can be powered by a 5 V USB power supply, either from a computer USB port or cell phone charging block, both of which are commonly available at low cost. The components' power requirements are small enough that the low-current digital pins on an Arduino can power them, allowing independent control of power to each component, which is leveraged in the implementation of power-saving features [62-64]. The scale's tare and calibration functions are controlled with a single push-button-pressed for tare and held for several seconds to calibrate.

As a logging scale, the microcontroller is configured to interface serially with a computer. The API for serial communication is built to comply with the Scale Manufacturers Association (SMA) SCP-0499 Level \#2 standard for limited-feature digital scale serial communication protocol. This includes capability to serially query the scale output, command tare, calibration, and provide basic scale information [65]. The API allows for continuous tracking of the scale readout for data-logging, which is useful for automated data-collection in cases such as long-term mass-tracking and discrete automated processes such as melt flow indexing (MFI).

\subsubsection{Bill of Materials}

The bill of materials (BOM) is shown in Table 2.1 .

Table 2.1. Visual bill of materials separated by mechanical and electrical components.

\begin{tabular}{c} 
Component \\
\hline 3-D Printed Components \\
Base \\
Print in normal orientation. Can be \\
printed without supports.
\end{tabular}




\section{Cover}

Print in either orientation. Can be printed without support, but this sacrifices top finish.

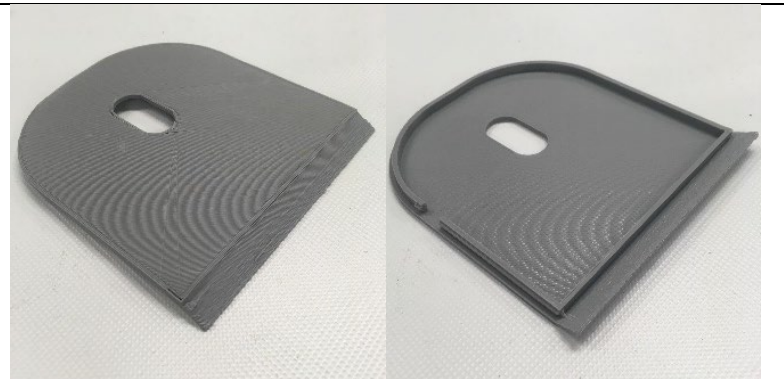

Bed

Print in either orientation. Can be printed without support, but this sacrifices top finish.

Cover (Optional)

Print upside-down without supports. This is used to reduce air currents for higher accuracy and precision.

\section{Electronic Components}

Arduino Microcontroller (Nano) $\$ 20.70$ [66]

(Derivative available with cable for $\$ 5.72[67])$

USB-A to mini-B USB (or micro, or USB-B, depending on the specific Arduino in use)

$\$ 5.26[68]$

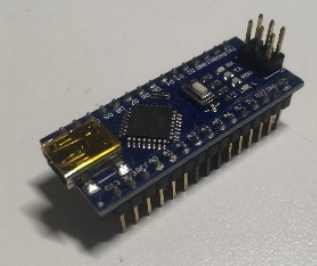


5 V USB Power Block (Optional)

$\$ 4.00[69]$

HX711 Loadcell Amplifier

$\$ 8.50$ with TAL220 [70]

Push-Button (Normally Open,

Momentary)

$\$ 2.50$ for 20 pack [71]

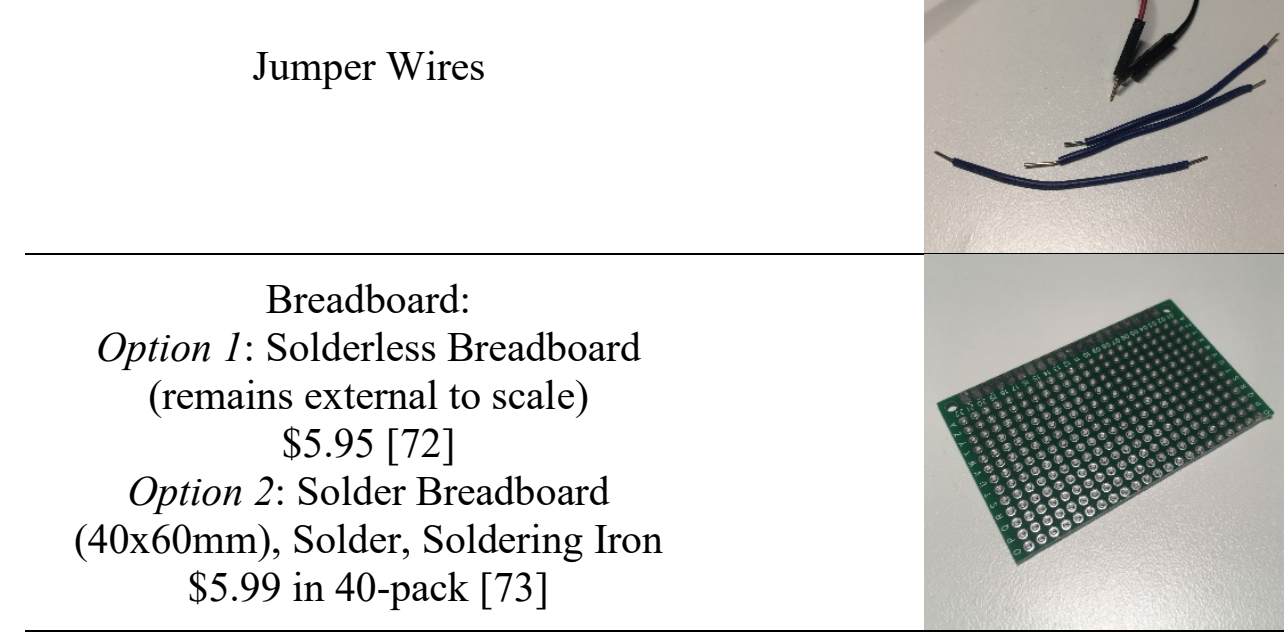

LCD is optional. The three components below are required if using an LCD. 


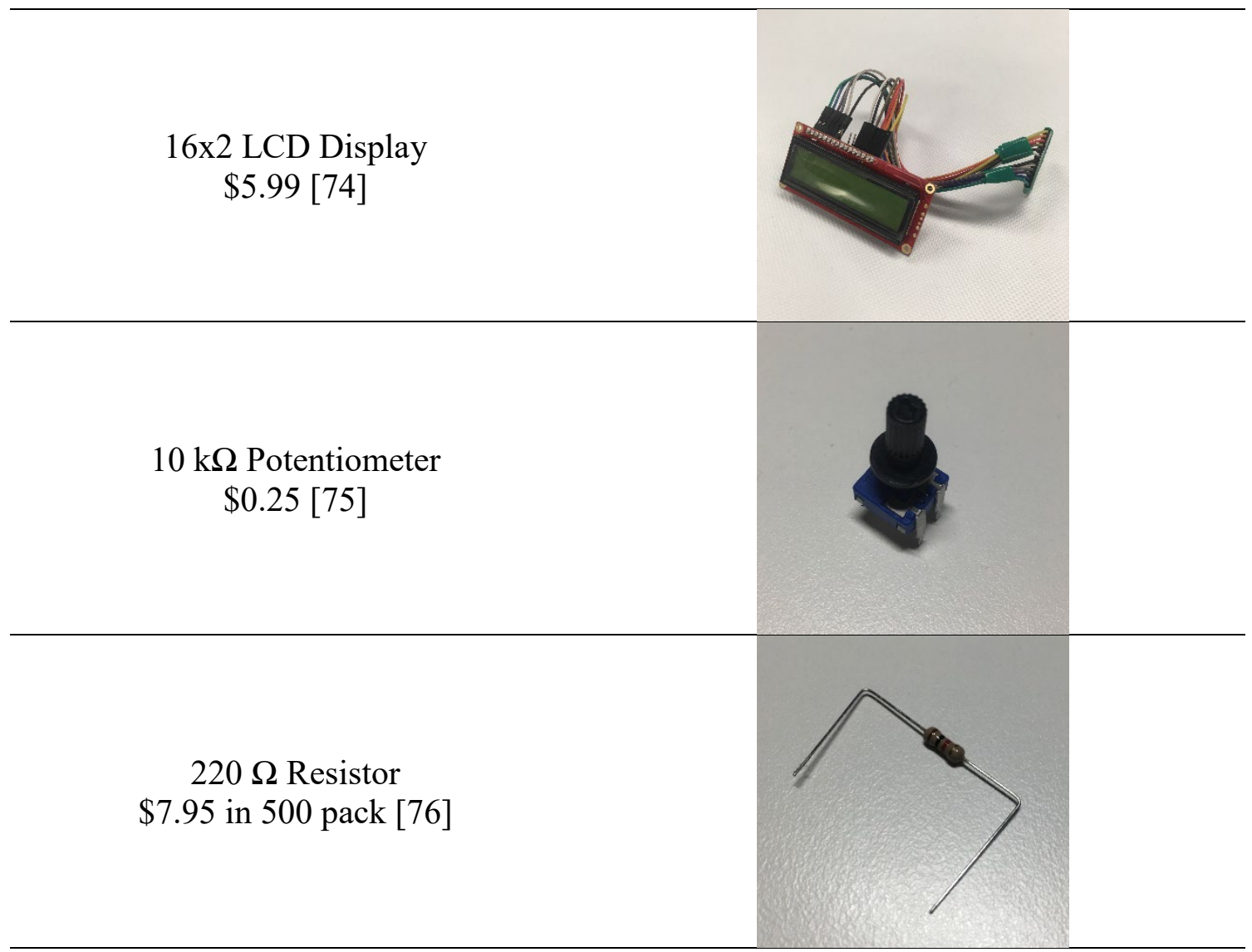

If using a solderless breadboard, no more electronic components needed. Using a solder breadboard:

Female Header Pins:

$1 \times 4(2)$

$1 \times 8(1)$

$1 \times 9(1)$

$\$ 0.95[77]$

Male Header Pins:

$1 \times 12(1)$

$1 \times 4(1)$

$\$ 3.00$ for $5 \times 16[77]$

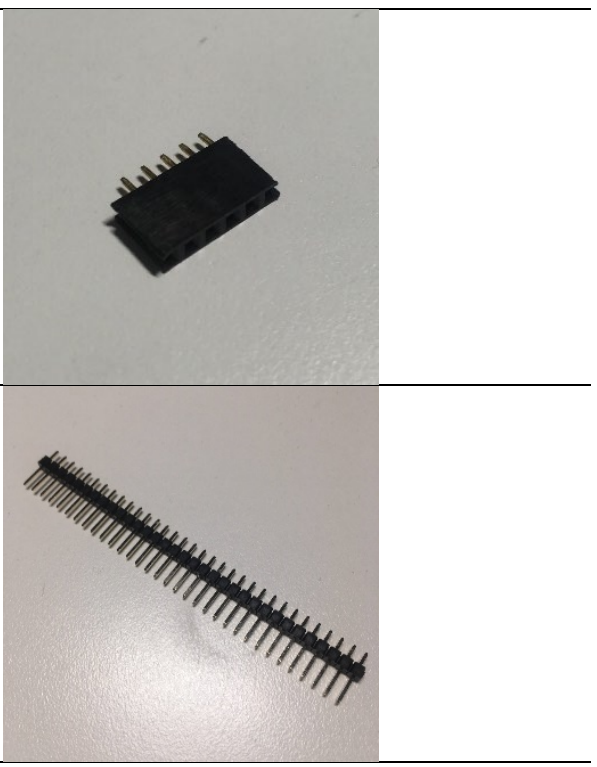

\section{Configuration Specific Hardware}

TAL 220 Loadcell configuration 


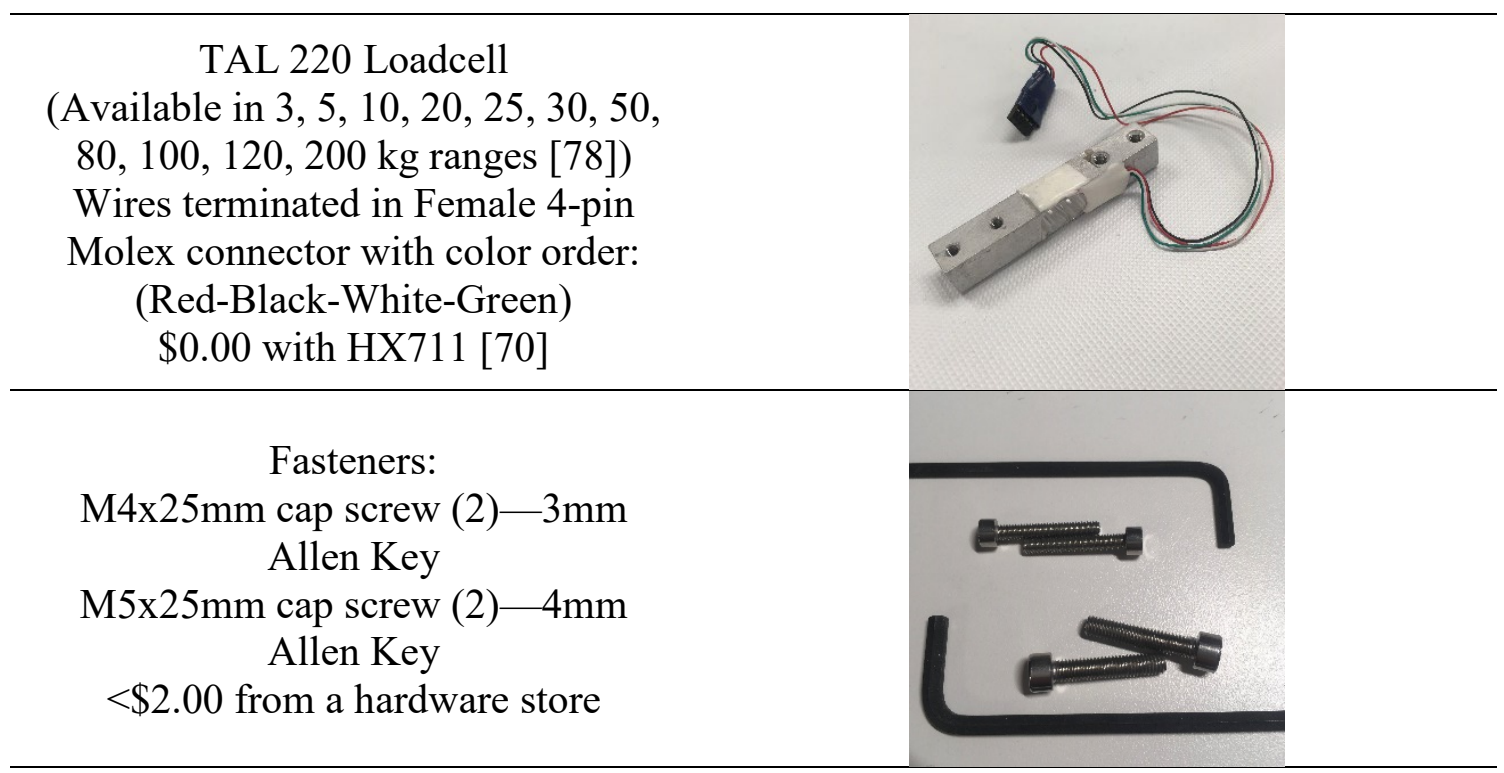

\section{TAL 221 Loadcell configuration}

TAL 221 Loadcell

(Available in 100, 150, 200, 300, 500,

750,1000 , and $1500 \mathrm{~g}$ ranges [79])

Wires terminated in Female 4-pin

Molex connector with color order:

(Red-Black-White-Green)

$\$ 8.95[80]$

Fasteners:

M3x20mm cap screw (4)-2.5mm

Allen Key

M3 nut (2) - 5.5mm Socket

$<\$ 2.00$ from a hardware store

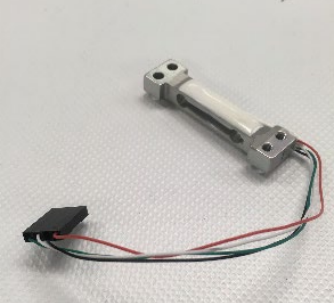

\subsubsection{Manufacturing and Assembly}

The 3-D printable components shown in Table 2.1 are available to be freely downloaded on the Open Science Framework (OSF) [81] and are released under a GNU General Public License (GPL) 3.0 [82]. All the required STL-rendered components were 3-D printed polylactic acid (PLA), filament of diameter $2.85 \mathrm{~mm}$ on a Lulzbot TAZ 6 (Aleph Objects, Loveland CO). The objects were sliced with Cura Lulzbot edition v.3.6.20 [83] using the settings detailed in Table 2.2. The optional cover was 3-D printed translucent glycol modified polyethylene terephthalate (PETG) filament of diameter $2.85 \mathrm{~mm}$ on a Lulzbot TAZ 6 [84]. 
Table 2.2. Print settings for the 3-D printed components.

\begin{tabular}{|c|c|c|}
\hline Property & PLA & PETG \\
\hline Layer Height & $0.14 \mathrm{~mm}$ & $0.18 \mathrm{~mm}$ \\
\hline Wall Thickness & $2 \mathrm{~mm}$ & $1 \mathrm{~mm}$ \\
\hline $\begin{array}{l}\text { Top/Bottom } \\
\text { Thickness }\end{array}$ & $2 \mathrm{~mm}$ & $1 \mathrm{~mm}$ \\
\hline Infill & Cubic, $20 \%$ & N/A \\
\hline Support Material & $\begin{array}{l}\text { Zigzag, Touching Bedplate Only, } 30 \%, 50 \\
\text { degrees }\end{array}$ & N/A \\
\hline Bed Adhesion & Skirt & Skirt \\
\hline Nozzle Temperature & $205^{\circ} \mathrm{C}$ & $230^{\circ} \mathrm{C}$ \\
\hline Print Speed & $\begin{array}{c}\text { Infill: } 40 \mathrm{~mm} / \mathrm{s}, \text { Wall: } 30 \mathrm{~mm} / \mathrm{s} \text {, Support: } 60 \\
\mathrm{~mm} / \mathrm{s}\end{array}$ & $\begin{array}{c}\text { Wall: } 20 \\
\mathrm{~mm} / \mathrm{s}\end{array}$ \\
\hline
\end{tabular}

After printing the components, all support material and brims were cleaned from the components. Please note that these components can all be printed without support material, depending on required surface finish.

The electronics were assembled according to the diagrams in Figure 2.1 and Figure 2.2, which were created with Fritzing [85]. The LCD was wired based on Arduino documentation [86].

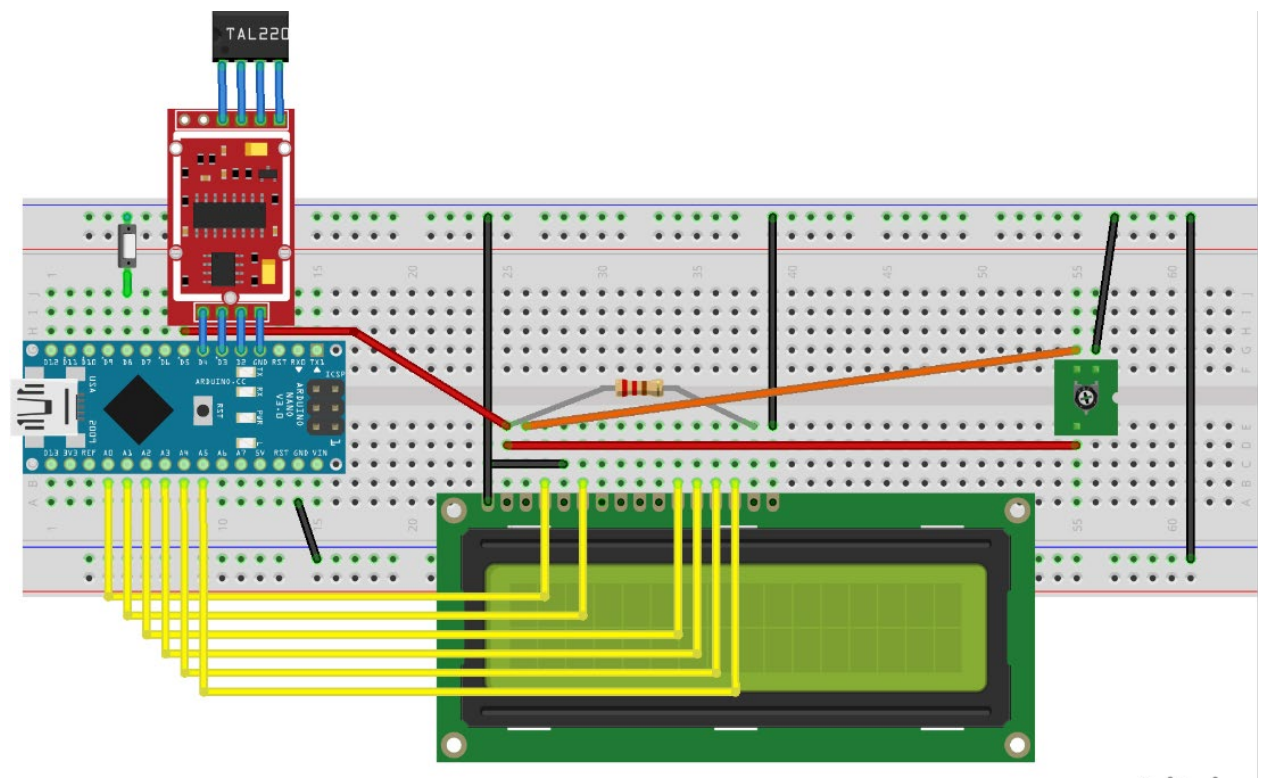

fritzing

Figure 2.1. Electrical circuit breadboard layout. Please note that all components connected to and including the LCD are optional. 


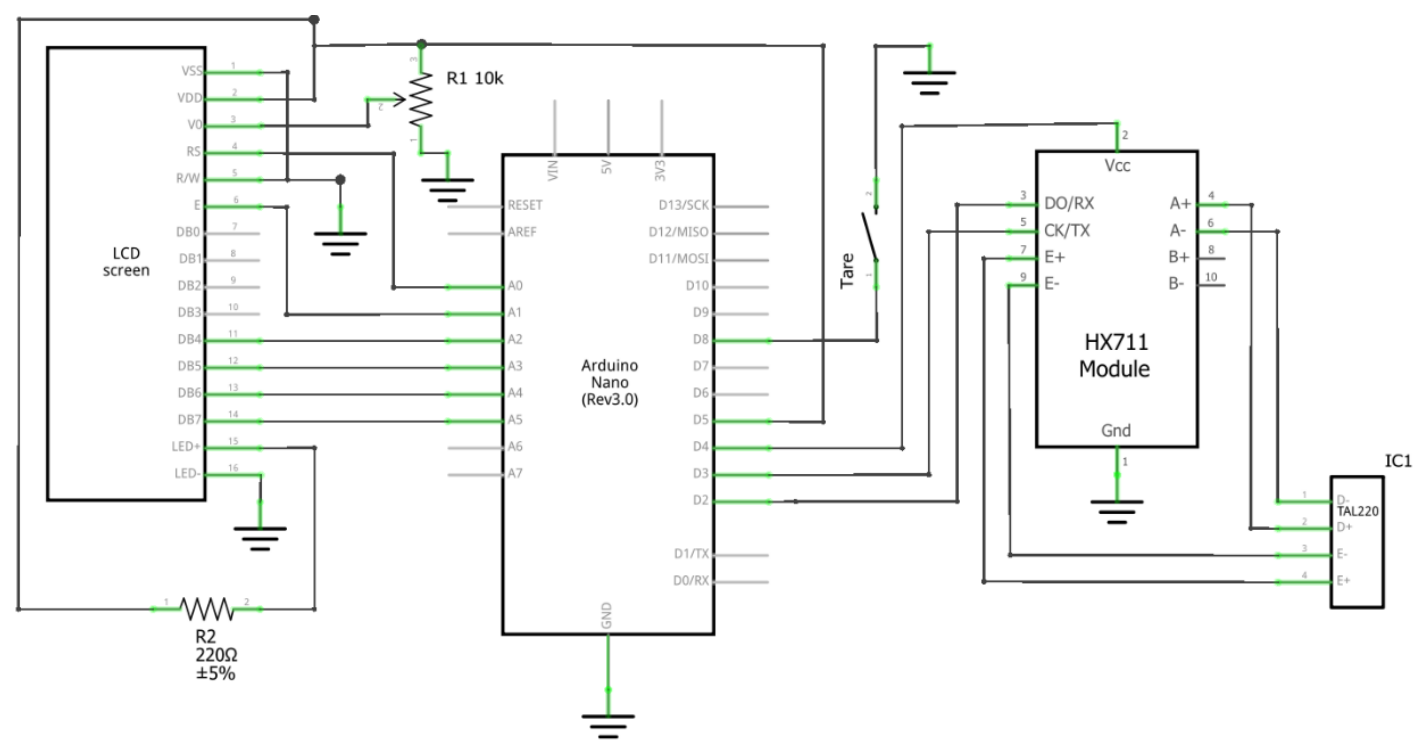

\section{fritzing}

Figure 2.2. Electrical circuit wiring diagram. Pinouts on the Arduino were selected to reduce the number of required jumper wires when using an Arduino Nano. Pinout selection can be modified in the firmware header file, Pinouts.hpp. Changing microcontrollers or pinouts may require different circuit parts than those listed in Table 2.1.

After verifying functionality with a solderless breadboard, the components were installed on a 40x60 mm solder breadboard as shown in Figure 2.3. To install the tare button on the face of the scale, a twisted pair was connected to the button pinouts and the button not immediately connected. The button was not connected to the twisted pair until after assembly. To allow for interchange of the Arduino, the HX711, the LCD, and the loadcell, these were connected with header pins and jumper wires. The Arduino was purposefully placed to align with the slot on the side of the 3-D printed base for USB access when fully assembled.

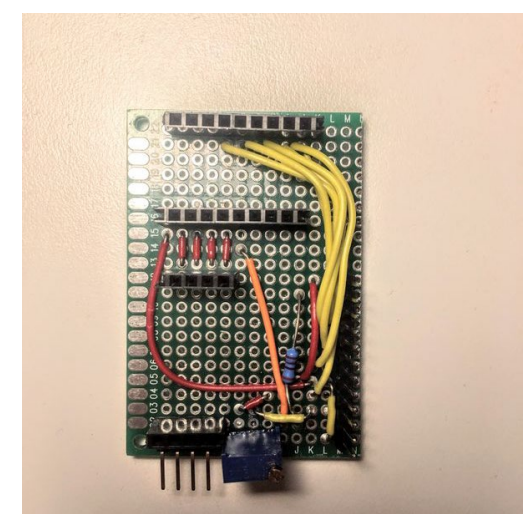

(a)

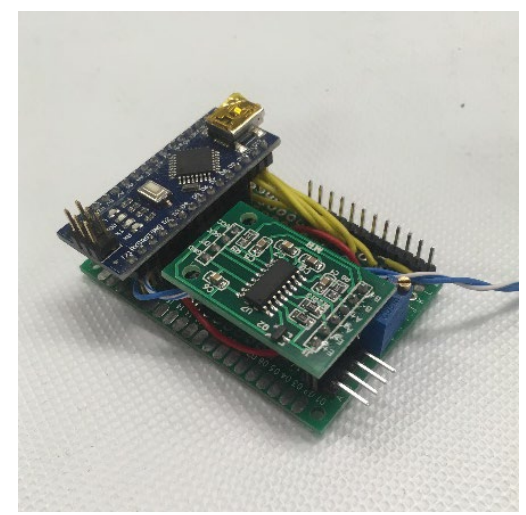

(b)

Figure 2.3. The circuit was assembled on a solder circuit board using jumper wires and header pins. The female header pins connect to the Arduino Nano and the HX711. The male header pins connect to the load cell and the LCD. The tare push-button is attached 
via twisted pair to allow installation on the face of the scale. (a) The soldered components were arranged in such a way to minimize wire use and to properly place the Arduino when installed in the base. (b) Shows the final circuit board.

The 3-D printed base has several features to aid in assembly (Figure 2.4). The assembled circuit board was fit into the 3-D printed base - the base has bosses on the right side (facing the front) that locate the board and hold it in place when connecting and disconnecting a USB cable to the Arduino (Figure 2.5).

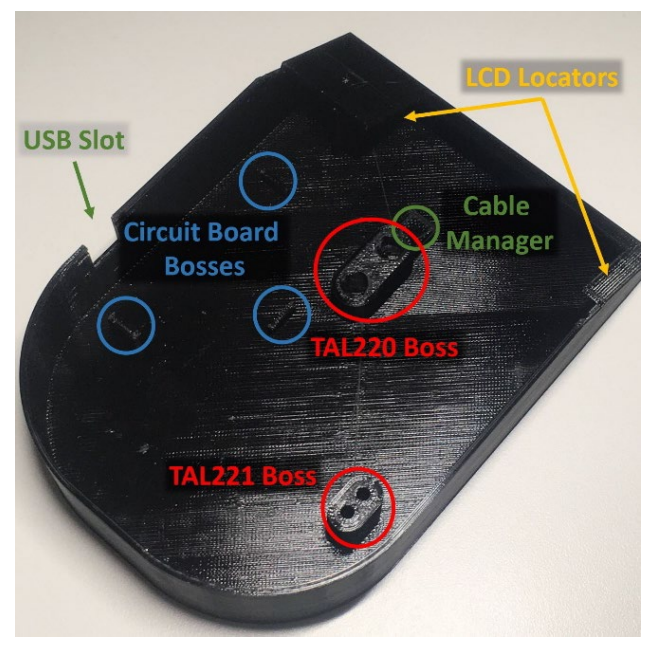

Figure 2.4. Key features of the base include standoffs to mount the load cell, locators for the screen and circuit board, and a slot for access to the Arduino Nano's USB port. The base also has a snap-seam to attach the cover to the base, enclosing the internal components.

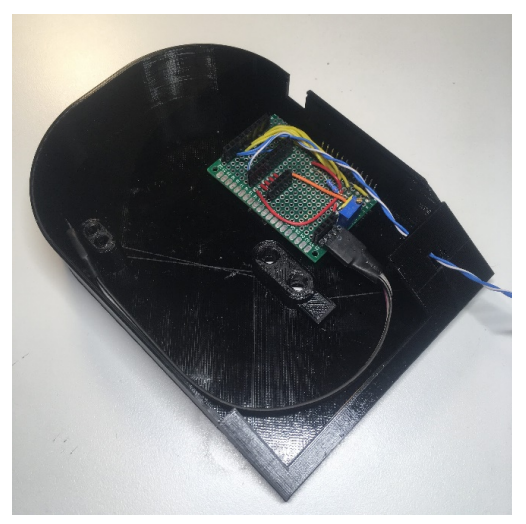

Figure 2.5. The circuit board fits into the bosses on the right side of the base to hold it in place. The twisted pair was fed through the hole for the push-button and a set of femalemale jumper wires were connected to the load cell header pins to reach the TAL221 wires.

The Arduino and HX711 were attached to their respective header pins. The LCD was placed in the locator slots on the front of the scale. The wires for the LCD were tucked 
under the cable manager on the TAL220 boss, then connected to the headers on the circuit board (Figure 2.6). The cable manager ensures that the wires from the LCD do not contact the loadcell, which would interfere with measurement accuracy.

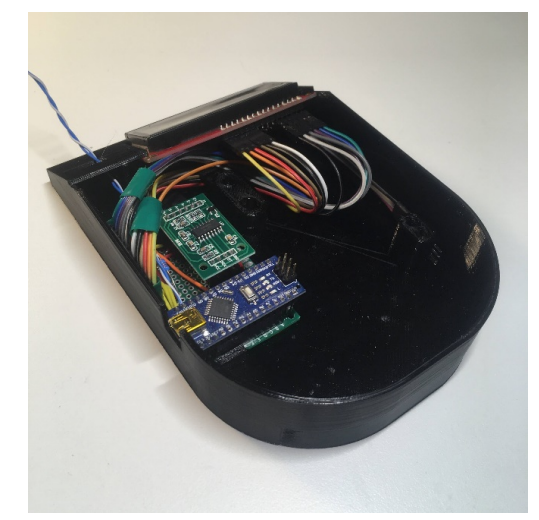

Figure 2.6. The LCD is held in place by two locators behind the slot for the screen. Its wires are tucked under a plastic wire manager attached to the TAL220 boss, then connected to the circuit board.

At this point the scale was powered on to set the potentiometer, which controls the contrast on the LCD. This can be set and then left alone. To do this, the Arduino was connected to a computer with the DigitalMassBalance firmware via USB. After uploading the firmware, a serial connection was opened to monitor the output from the microcontroller. The output indicates whether the scale has initialized correctly, informing the user as devices are powered on and checked. The LCD continuously displays the measured mass once powered on. The potentiometer was adjusted until the readout (number and unit) were easily visible on the LCD. In cases that the display appears non-functional, it should be checked in low light - if the screen does not appear backlit, no power is reaching the LCD. If it is receiving power, one of the jumpers is likely loose and all connections should be checked. SparkFun's LCD Hookup guide provides helpful and detailed troubleshooting suggestions if these checks do not work [87].

Prior to soldering the push-button in place, the lead wire connections to the controller should be checked. With the controller powered on, the tare is checked by shorting the twisted pair - a dot display on the bottom right of the LCD appears when the pair is shorted, indicating proper communication. With that checked, the button can then be soldered and secured in place. A small amount of non-conductive glue may be used to keep the button in place.

After setting up the LCD, the loadcell was installed. The loadcell must be mounted so that its wires run toward the base-fixed end of the loadcell. The wires will interfere with measurement accuracy if running from the floating end of the loadcell. The installation procedure depends on the loadcell style in use: 
- TAL220: Install the M5 end (the wires run to this end) to the TAL220 boss on the base of the scale (Figure 2.7). Connect the loadcell to the HX711 pinoutsRed:E+, Black:E-, White:A-, Green:A+. Snap the cover onto the base- this fully encloses the electronics, offering some protection from thermal variations on the amplifier. Attach the bed to the loadcell using the M4 bolts.

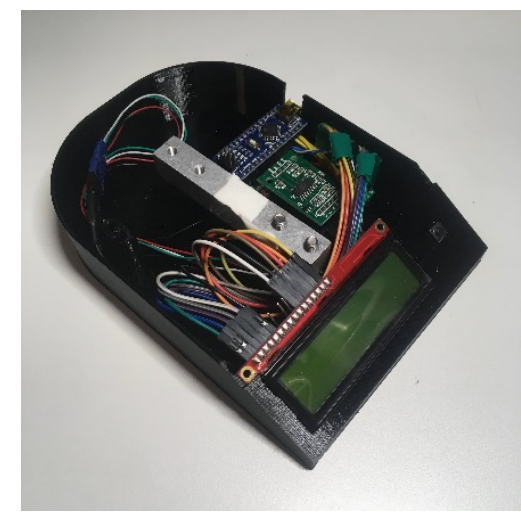

(a)

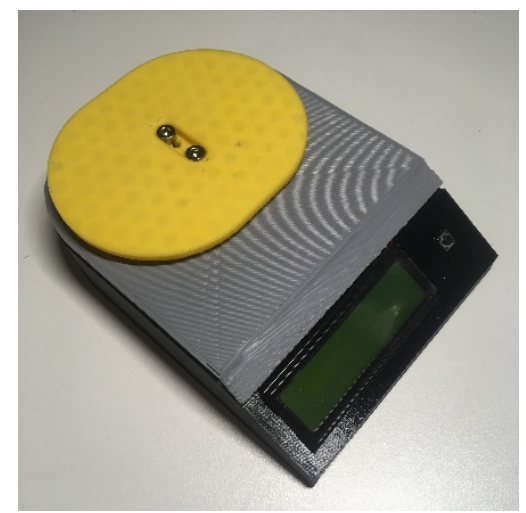

(b)

Figure 2.7. (a) The TAL220 loadcell should be installed with the wires leading off the end of the loadcell attached to the base - the M5 tapped holes. Please note that the button is also installed. (b) After snapping on the lid, the bed can be attached to the free end of the load cell using two M4 bolts.

- TAL221: Sandwich the cover between the bed and the loadcell. Connect the untapped end of the loadcell (the wires do NOT run to this end) to the bed using M3 screws and nuts (Figure 2.8a). Connect the loadcell to the HX711 pinoutsRed:E+, Black:E-, White:A-, Green:A+. Finally, attach the tapped M3 end to the TAL221 boss on the base (Figure 2.8b). This fit is tight so the wires on the loadcell lead off the end fixed to the base

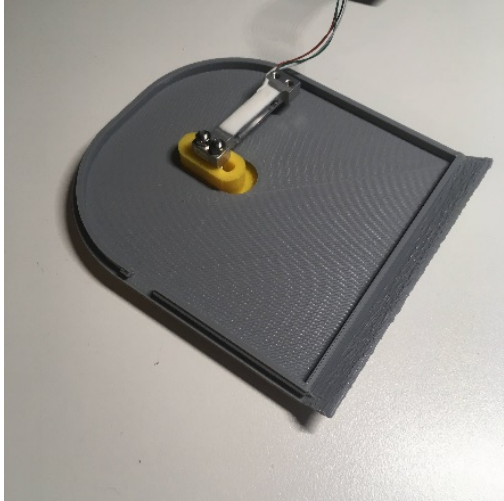

(a)

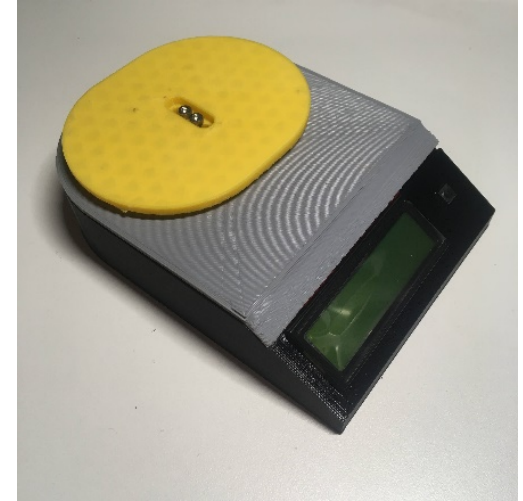

(b)

Figure 2.8. (a) The cover must be sandwiched between the loadcell and the bed prior to attaching to the base. Note the direction the loadcell is aimed so it will rest on the TAL221 boss when the cover is snapped on. (b) After connecting the loadcell to the controller, the cover is snapped on and the TAL22 1 is screwed to the base using two M3 bolts. This must be done by feel, but does not take very long. 


\subsubsection{Firmware}

The firmware to drive the scale (called DigitalMassBalance) is also in the OSF repository [81]. A single installation contains functionality for both configurations of the scale (simple scale with digital display and lab scale with serial interface). The configuration header file, Config.hpp, can be modified to tell the controller whether an LCD is installed. Changing this setting determines whether the LCD will be initialized upon startup; and the serial interface listens by default as this has negligible effect on performance.

The firmware package makes use of two open source libraries: (1) the HX711 library by GitHub user bogde [88], used under the MIT License; and (2) the LiquidCrystal Library for Arduino by Hans-Christoph Steiner [89], used under the GNU Lesser General Public License.

The HX711 library provides an interface with which to initialize, and read from, the HX711 loadcell amplifier. The HX711 library can provide raw (long integer with tare offset) or calibrated (scaled by a sensitivity value from unitless integer to mass with units) data. The library's built-in tare offset is used to implement zeroing of the scale (only the bed - no containers), while taring (subtracting a known mass from the reported value) is implemented in the firmware. Raw data is collected from the HX711 library to allow flexibility in the data averaging/filtering scheme used by the scale.

The LiquidCrystal library is used to write information to the LCD. Methods in the DigitalMassBalance firmware interface with the library, clearing the screen, moving the cursor, and writing information.

The DigitalMassBalance firmware itself is composed of a single Arduino source-code file, DigitalMassBalance.ino, which implements all methods and loops used during normal operation, plus three header files which organize definitions used by the firmware:

- Config.hpp contains configuration variables for the HX711, the LCD, and calibration and serial communication protocols.

- Libraries.hpp contains '\#include' statements for all the libraries used by DigitalMassBalance.

- Pinouts.hpp contains definitions for the location of hardware connections to the Arduino. These can be modified to accommodate the pinouts used in a particular setup.

Upon receiving power, the Arduino runs its setup loop, which completes six steps:

- Initialize a 9600 baud serial connection. The firmware is set to wait for serial communication to initialize before continuing. Please note that this does not noticeably affect startup time when receiving power from a non-serial-enabled device (such as a $5 \mathrm{~V}$ power block or a battery). 
- The HX711 undergoes a similar startup procedure, receiving power and ensuring proper communication. This is indicated by a series of readouts over serial.

- If an LCD is expected by the firmware (set in Config.hpp), the LCD is powered up. Once it is on, all digits of the display flash with the character ' 8 ' for less than a second. This initialization is also indicated by readouts over serial.

- With the hardware running, the Arduino checks its hard memory (EEPROM) for a saved calibration sensitivity (such a value is saved at the end of a calibration sequence). If one exists, the sensitivity is read and applied to the digital readout. If no sensitivity is saved, the scale defaults to a value of 1 , which just returns the raw measurement from the HX711. The read sensitivity value is reported over serial. This message completes unsolicited responses from the scale-all further communication from the Arduino result from commands sent over serial. A complete, successful initialization is shown in Figure 2.9.

- At the time of writing, a simple averaging window is used to stabilize measurements from the scale. Measurements are stored in a first-in-first-out (FIFO) queue whose size is set in Config.hpp. The size of this window affects the response time of the scale. During initialization, this queue is automatically cleared to an array of zeros.

- Finally, the tare button is set to an input in INPUT_PULLUP mode, which makes use of an internal pullup resistor. This setting saves component cost and makes the tare button active LOW.

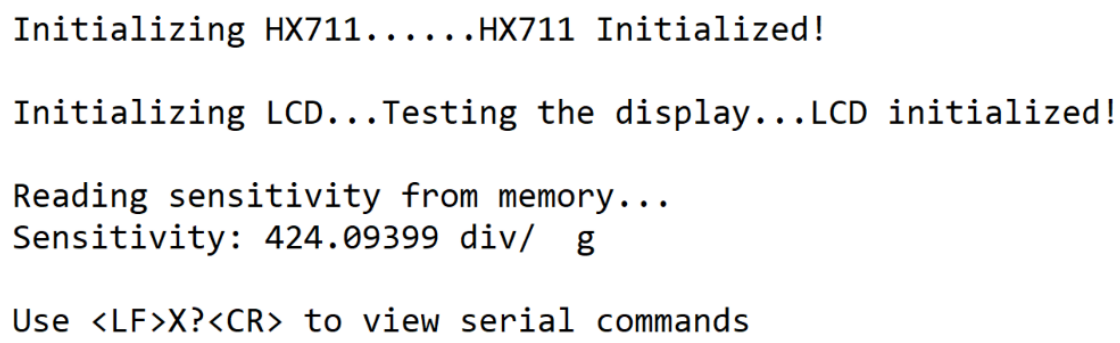

Figure 2.9. The scale returns a readout similar to the one shown during initialization. The readout indicates the action in progress, success, and returns the sensitivity value when reading from memory.

After completing setup, the Arduino enters a loop. With each iteration, the current mass measurement is retrieved from the HX711 and stored in the averaging queue, and the average mass is calculated and stored. This occurs as fast as the Arduino can processthere is no enforced sample rate for the mass. The Arduino also listens for serial input consistent with SMA SCP 0499 (detailed in 2.2.5.2, which discusses the operation of the scale as a lab scale) [65]. At the same time, it displays the current averaged mass measurement on the LCD and checks for a press of the tare button. These listening and reporting actions are completed at an enforced refresh rate (set in Config.hpp). This is done to visually stabilize (slow down) the LCD output and to enforce the rate at which data is reported over serial. 


\subsubsection{Operation of Design}

The scale is designed for two forms of interaction: (1) as a simple scale with LCD output and push-button interaction and (2) as a lab scale with a serial interface to allow datalogging and advanced control of the scale. Both are discussed below as well as illustrated in 'MOST OS Balance.mpeg', a use video available on the OSF repository.

\subsubsection{Simple Scale}

For simple use, the scale is connected to power over USB to a $5 \mathrm{~V}$ power block, such as that used to charge a cellphone. Alternatively, $5 \mathrm{~V}$ power can be provided to the Arduino $5 \mathrm{~V}$ and Ground (GND) pins through other means. Possible interactions are:

- Tare: A press and release of the push-button on the front of the device will tare the scale to the current weight. The push-button is acknowledged when closed (pushed) by a dot on the lower right-hand corner of the LCD.

- Calibrate: Pressing and holding the push-button for at least 3 seconds (the wait time is adjustable in Config.hpp) will initialize a calibration sequence using the calibration mass set in Config.hpp. The scale will tare, then indicate the mass to apply to the bed. After detecting an added mass, the scale will measure the mass for 10 averages, then calculate the new scale sensitivity and save it to EEPROM.

\subsubsection{Lab Scale with Serial Interface}

The scale's 9600 baud serial interface has been designed to Level \#2 compliance with the SMA Scale Communication Protocols [65]. This includes the six specified Level \#1 commands, plus additional commands. The full command list is detailed below, listing the command name, command character (indicated $<$ char $>$ ), followed by a brief description where necessary. All command characters must follow a linefeed $<\mathrm{LF}>$ and precede a carriage-return $<\mathrm{CR}>$. Full details of the response format and communication protocol are available in SMA SCP-0499 [65].

- Return displayed weight $<\mathrm{W}>$.

- Zero the scale $<\mathrm{Z}>$.

- Run scale diagnostics $<\mathrm{D}>$. This is specified by SMA to check for memory or calibration errors.

- Return 'about' information for the scale $<\mathrm{A}>,<\mathrm{B}>$. These are specified by SMA to return information about the scale's compliance level and firmware information.

- Reset the scale $<$ ESC $>$.

- Continuously report weight $<\mathrm{R}>$. Returns weight at the read rate until another command is received.

- Tare the scale $<\mathrm{T}>$. This is different from zeroing in that the tare is for, as an example, ignoring the mass of a container. Meanwhile, zero is intended to set 'zero' for the scale with nothing on the bed.

- Clear the current tare $<\mathrm{C}>$. This sets the tare weight to zero, causing further readouts to reference to the zero set by $<\mathrm{Z}>$ 
- Return the tare weight $<\mathrm{M}>$.

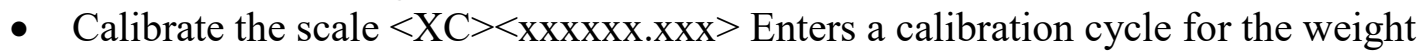
specified by $<\operatorname{xxxxxx.xxx>}>\operatorname{XC}>$ on its own may also be used to enter a calibration sequence using the default calibration mass set in Config.hpp

- Toggle power to the $\mathrm{LCD}<\mathrm{XL}>$. This changes the power state of the LCD, either turning it off or reinitializing it. This can be used for power savings when the display is not needed.

- Scroll output precision $\langle\mathrm{XP}>$. This scrolls the number of digits on the readout between 0 and 4 .

- List possible commands $<\mathrm{X}$ ? $>$. This lists the recognized commands for the user. Since the leading X (required by SMA SCP 0499) is non-intuitive, it is indicated at the completion of the setup loop (Figure 2.9).

\subsubsection{Validation}

The 3-D printed open source scale was tested against two proprietary laboratory-grade scales and with standard calibration weights, to check the functionality, accuracy, and linearity of the scale. The open source scale was tested for three loadcells: 1) a $5 \mathrm{~kg}$ TAL220 loadcell, 2) a $500 \mathrm{~g}$ TAL221 loadcell and 3) a $100 \mathrm{~g}$ TAL221 loadcell. TAL220 and TAL221 refer to the shape of the load cell. The mass range is determined by the size of the cutout in the middle of the loadcell where less material left behind yields a more sensitive/lower mass range.

\subsubsection{Laboratory-Grade Scale Comparison}

The open source scale was tested against a Denver APX402 400/10 mg digital mass balance and a Denver A-160 160/100 $\mu \mathrm{g}$ digital analytical balance [90,91]. The A-160 was calibrated 2 months prior to the first round of testing. Due to limitations in the range of the two proprietary lab-grade balances, neither loadcell was tested to the full range in this scale. The APX402 and the open source scale were tested up to $300 \mathrm{~g}$. The A-160 and the open source scale TAL221 were tested up to $150 \mathrm{~g}$. However, rigorous testing of the loadcells themselves, was not the purpose of this testing - the results of such testing are documented in the manufacturer data sheets for loadcells and the loadcell amplifier [63; 78-79].

To calibrate the scale, a container was measured on the A-160. Using the measured mass (reported down to $100 \mu \mathrm{g}$ ) as a reference value, the scale was put into calibration mode. The scale tared itself, then waited for the reference mass to be added. Once added, the scale took a total of 100 averaged measurements over the span of several seconds in order to determine the sensitivity of the loadcell (i.e., calibration value that multiplies a raw value to obtain a value with units).

The tests were completed using a light container with sand incrementally added. The container was placed on the APX402 scale, but not tared. Sand was added to the container until a desired mass was achieved. The container of sand was then massed on the APX402, the A-160, and the scale, in that order. After the upper limit of the A-160 was reached $(160 \mathrm{~g})$, the APX402 and open source scale were still tested until the upper 
limit of the APX402 (around $330 \mathrm{~g}$ ). The A-160 has a glass case, which was closed while taking measurements. The APX402 and the open source scale did not have any cases to protect from airflow interference. Measurements were repeated 5 times for each increment of mass to check the repeatability of measurements (precision).

To compare the measurements among the three scales, two measures were computed. First is the standard deviation of the measurements at each discrete mass. This was computed for each individual scale to offer comparison on the stability of their readouts, indicating the precision of each instrument. Second, in an attempt to quantify the accuracy of the scale, its average measurement for each discrete mass up to $160 \mathrm{~g}$ was compared to the average measurement of the A-160 (the device used to calibrate it) using the absolute value of the difference between the two. The differences offer some perspective on how closely the open source scale tracked with the proprietary lab-grade scales.

\subsubsection{Self-Calibration of Open Source Scale for $100 \mathrm{~g}$ TAL221}

Often a developer of open hardware may only have access to a calibrated scale initially, so it is important to be able to calibrate the open source scale from previous measurements (e.g., self-calibration). To demonstrate this the $100 \mathrm{~g}$ TAL221 was tested against the previously tested (calibrated) $500 \mathrm{~g}$ TAL221.

Four containers were filled to nominal masses of $25,50,75$, and $100 \mathrm{~g}$ as measured on the $500 \mathrm{~g}$ TAL221. These new masses can be considered secondary standards as their masses are known to the accuracy of the $500 \mathrm{~g}$ TAL221. After measuring all 4 masses 5 times on the scale, the $100 \mathrm{~g}$ TAL221 was installed on the open source scale. It was calibrated using the $75 \mathrm{~g}$ mass. All 4 masses were then measured 5 times on the $100 \mathrm{~g}$ TAL221. The resulting data was processed in the same manner as the other two tests, except average differences are referenced to the $500 \mathrm{~g}$ TAL221 loadcell.

\subsubsection{Measurement on Open Source Scale Using Standard Masses}

In a final set of tests to quantify the behavior of each load cell, the open source scale was used to weigh a set of calibration weights (standard masses) ranging from $1 \mathrm{~g}$ up to $100 \mathrm{~g}$, with rated accuracy of $+/-5 \mathrm{mg}$. The scale was set up with each load cell installed in the 3 -D printed (PLA) housing and connected via USB to a computer. With the scale powered up, the electronics were allowed to warm up for at least one hour prior to calibration and testing.

The masses were handled using tweezers and stored in a plastic bag in between measurements to prevent contamination (which could affect the overall weight). The 100 $\mathrm{g}$ TAL221 was calibrated using a $20 \mathrm{~g}$ mass; the $500 \mathrm{~g}$ TAL221 and $5 \mathrm{~kg}$ TAL220 were calibrated using a $100 \mathrm{~g}$ mass. The sensitivities used for each load cell were recorded with the raw data.

A PuTTY session was used to log data returned from the scale in continuous logging mode (command $<\mathrm{R}>$ ). The scale was set to report an averaged weight at a report rate of 
$1 \mathrm{~Hz}$. The average weight was calculated from a 10-value sliding average, sampled at an unregulated rate (however fast the processor could loop). This was observed to have a $10-90 \%$ rise time of 1 second. Each mass was placed on the scale and allowed to sit for around 30 seconds, returning between 23 and 48 measurements (depending on the actual time the mass was on the bed). Two complete datasets were gathered and averaged together, providing a minimum of 48 measurements for each mass on each load cell.

The standard deviation and average value of the logged data were calculated. The raw and processed data are stored in the OSF repository [81]. These results illustrate the scale's behavior (statistical dispersion) without the influence of a human reading numbers from a display. It also gives a better indication of the accuracy of the scale since the masses are considered to be known.

To assess the influence of the plastic housing on the scale's behavior, the $5 \mathrm{~kg}$ TAL220 was re-tested while mounted to a simple frame made of two 1x6x6 inch $(25 \times 150 \times 150$ $\mathrm{mm}$ ) common pine boards with spacers to float the load cell body above the pine, providing a fourth dataset for comparison.

\subsubsection{Economic Analysis}

To determine the costs of the various versions of the open source digital scale, the 3-D printable components were massed on another digital scale with precision $+/-10 \mathrm{mg}$. The total cost (Tc) of the apparatus can be determined by

$$
\mathrm{Tc}=\mathrm{V}+\mathrm{mCe}+\mathrm{mCp}
$$

Where $\mathrm{V}$ is the cost of the vitamins (or non-digitally manufactured components listed in Table 2.1), $\mathrm{m}$ is the mass of all the 3-D printed parts (e.g., all the STL-rendered parts in Table 2.1); Ce is the cost of the electricity per kg to print; and $\mathrm{Cp}$ is the cost of plastic per $\mathrm{kg}$. The Lulzbot Taz 6 uses approximately $9.11 \mathrm{kWh}$ per $\mathrm{kg}$, as measured by a multimeter $+/-0.01 \mathrm{kWh}$ and reported previously [92]. The average cost of electricity in the U.S. is about $\$ 0.1029 / \mathrm{kWh}[93]$. The cost of the PLA filament from Lulzbot was U.S. $\$ 20 / \mathrm{kg}$ [94].

\subsection{Results}

Two sets of testing were completed on the scale. These tested the scale under different conditions and offer different insights to the behavior of the scale.

The first set of tests compares the open source scale with commercial scales. This set trusts the commercial scales to provide an accurate weight measurement, both for calibration and comparison. These tests exercise the repeatability of the scale when removing and re-adding weight to the scale. The measured mass was recorded by reading the value from the LCD, introducing room for human error during the test.

The second set of tests use only the open source scale to measure standard calibration weights. This set trusts the weights to be accurate for calibration and for assessing the accuracy of the scale. The measured mass was recorded automatically via serial 
communication, providing a larger data set to illustrate the motion of the scale's readout when at rest. Since the masses are assumed to be known, this set gives a better idea of the accuracy of the scale (within the limits of the accuracy of the masses themselves).

\subsubsection{Laboratory-Grade Scale Comparison}

Repeatability testing on the $5 \mathrm{~kg}$ TAL220 loadcell yielded a standard deviation of 16.3 $\mathrm{mg}$ on average ( $\max 28.8 \mathrm{mg}$ ), as compared to $3.5 \mathrm{mg}$ for the APX402 and $200 \mu \mathrm{g}$ for the A-160. The average absolute difference between the open source scale and the A-160 was $36.3 \mathrm{mg}$. The standard deviation and absolute differences are plotted for each discrete mass in Figure 2.10a and Figure 2.10b, respectively.

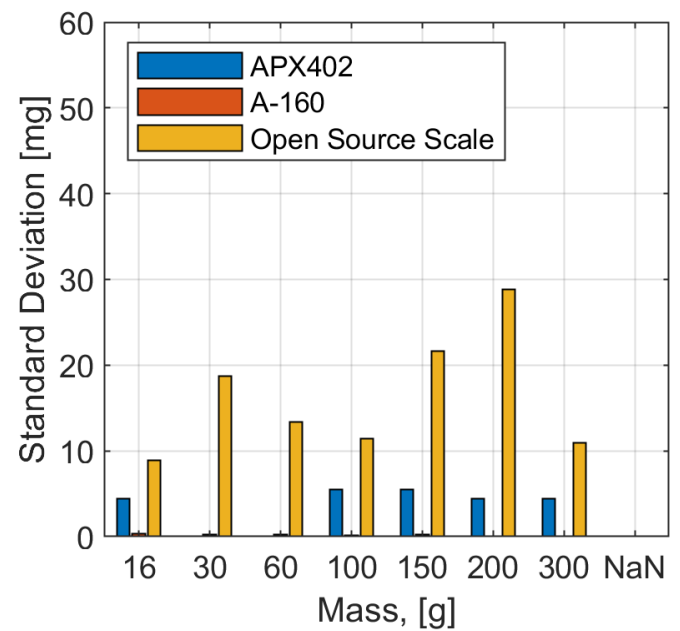

(a)

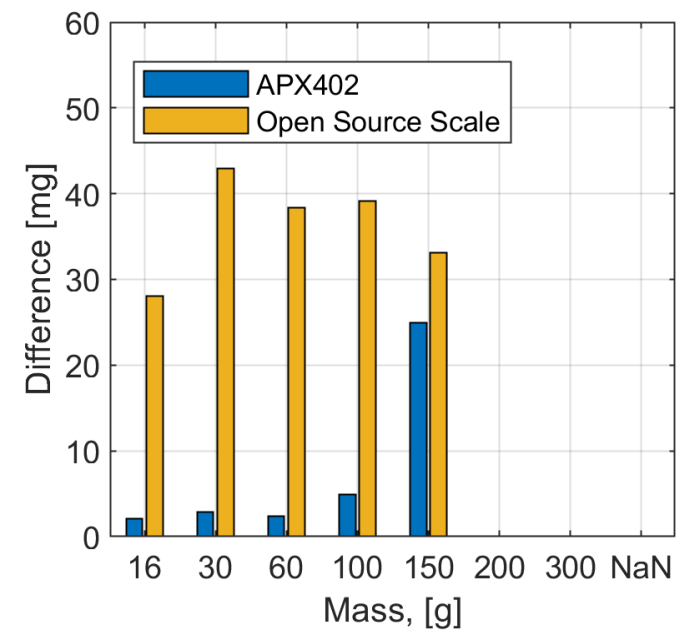

(b)

Figure 2.10. The $5 \mathrm{~kg}$ loadcell results are shown (a) The standard deviation (sample of 5 measurements) averaged to $16.3 \mathrm{mg}$ for the open source scale. This is large relative to the APX 402 and the A-160. The A-160 performed at a very small standard deviation relative to the other scales, causing its bars to be difficult to see at the bottom of the chart; (b) The absolute value of the difference between each scale and the A-160 is shown. The open source scale averaged a difference of $36.3 \mathrm{mg}$.

Testing conducted on the $500 \mathrm{~g}$ TAL221 loadcell yielded a standard deviation of $20.7 \mathrm{mg}$ on average ( $\max 52.1 \mathrm{mg}$ ), as compared to $4.3 \mathrm{mg}$ for the APX402 and $2.7 \mathrm{mg}$ for the A160. The average absolute difference between the open source scale and the A-160 was $14.2 \mathrm{mg}$. The standard deviation and absolute differences are plotted for each discrete mass in Figure 2.11a and Figure 2.11b, respectively. 


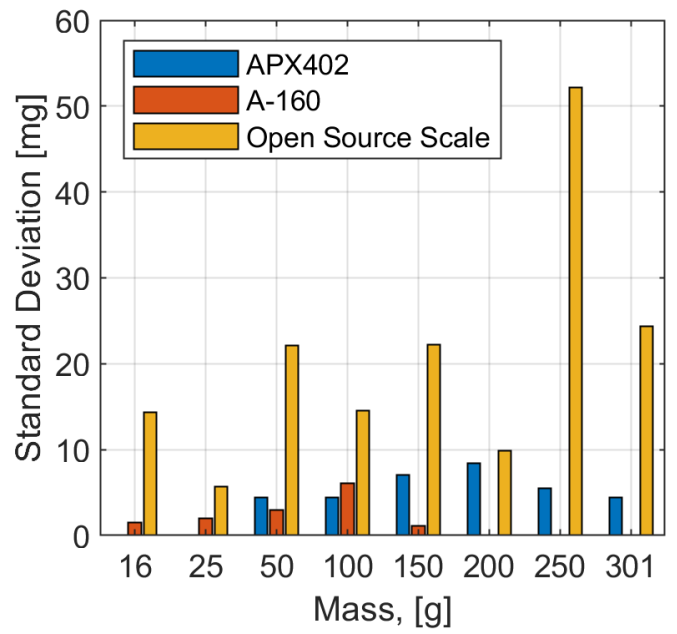

(a)

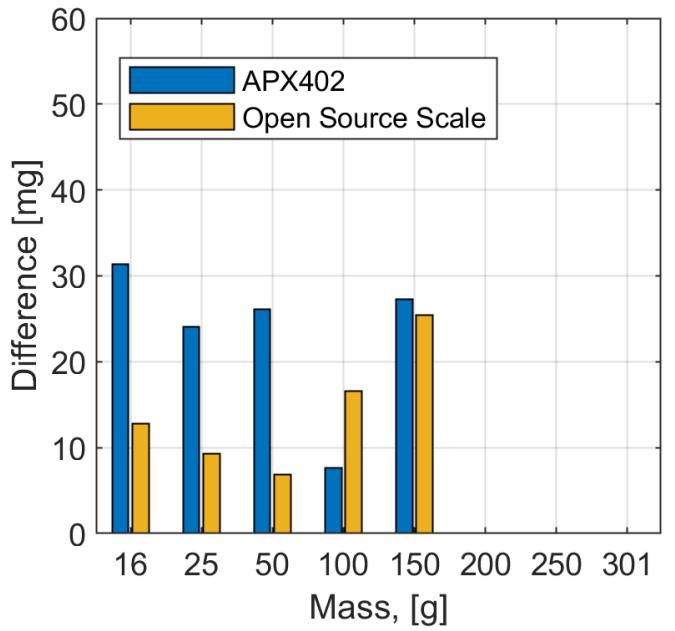

(b)

Figure 2.11. The $500 \mathrm{~g}$ loadcell test results are shown. (a) The standard deviation (sample of 5 measurements) averaged to $20.7 \mathrm{mg}$ for the open source scale. This is large relative to the lab-grade scales; (b) The absolute value of the difference between the average measurements by the A-160 and the other two scales are shown. The open source scale average measurement was within $14.2 \mathrm{mg}$ of the A-160, on average.

The testing completed on the TAL221 $500 \mathrm{~g}$ loadcell followed the same procedures, but the conditions appear to have been different. The A-160's standard deviation was an order of magnitude higher than during testing of the TAL220, and the difference between the APX402 and the A-160 was 5 times larger, on average. It is expected that the environment had some effect on testing - it is possible that the ventilation system could have affected measurements by increasing airflow on the scales. It may also be the case that the scales were not properly pre-warmed (powered on for 15 minutes to an hour) prior to the initiation of testing.

Finally, testing conducted on the $100 \mathrm{~g}$ TAL221 yielded a standard deviation of $5.0 \mathrm{mg}$ on average (6.6 $\mathrm{mg} \max$ ). This is around $20-50 \%$ higher than the standard deviation measured for the APX402 during the two rounds of testing detailed above, indicating the $100 \mathrm{~g}$ loadcell offers precision on the order of (though slightly lesser than) the APX402. The average difference between the $100 \mathrm{~g}$ and $500 \mathrm{~g}$ TAL221 loadcells was $19.8 \mathrm{mg}$ on average, though the average difference here should not be taken alone to certify the accuracy of the $100 \mathrm{~g}$ loadcell, as there is unquantified error propagation between the calibration of the $500 \mathrm{~g}$ and $100 \mathrm{~g}$ loadcells. This is the limitation of using the open source scale to make secondary calibrated masses. The error propagation is a result of the calibration of the $100 \mathrm{~g}$ TAL221 against the $500 \mathrm{~g}$ TAL221, rather than the A-160. The test results are shown in Figure 2.12. 


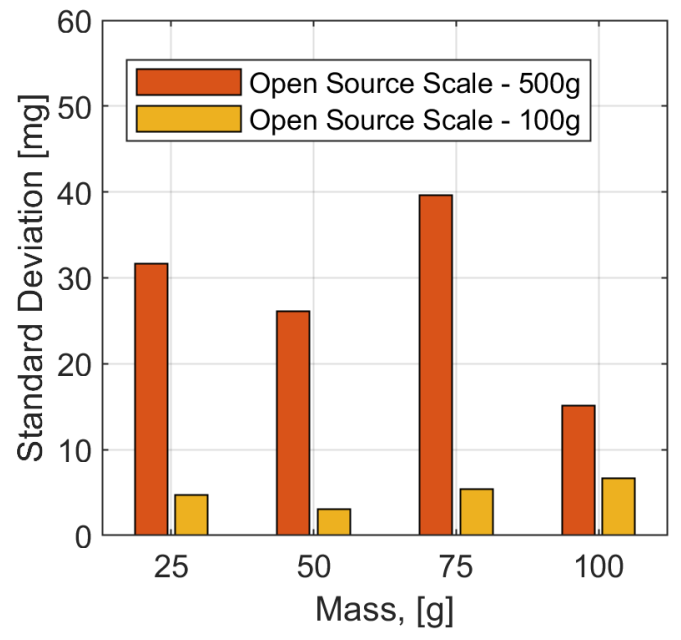

(a)

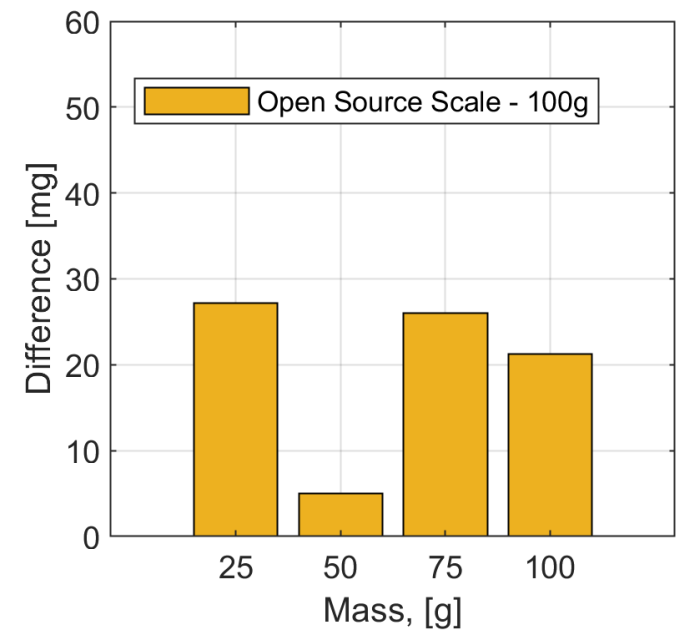

(b)

Figure 2.12. The $100 \mathrm{~g}$ loadcell test results are shown. (a) The standard deviation (sample of 5 measurements) averaged to $5.0 \mathrm{mg}$ for the open source scale. This is close to that of the lab-grade scales (on the same order as the APX402); (b) The absolute value of the difference between the average measurements by the $500 \mathrm{~g}$ TAL221 and the $100 \mathrm{~g}$ TAL221 are shown. The $100 \mathrm{~g}$ TAL221 average measurement was within $19.8 \mathrm{mg}$ of the $500 \mathrm{~g}$ TAL221, on average.

These tests showed that the scale tracks linearly with the two lab-grade scales (i.e., the measurement does not become more or less accurate as mass increases), but its output is less precise. The precision of the scale is a function of the range and style of the loadcell. These results support the idea that smaller range loadcells produce more precise measurements, with the $100 \mathrm{~g}$ TAL221 approaching the precision of the APX402. The average standard deviation (Isigma) of each loadcell tested is compared to its range in Table 2.3 as a percentage $\left(\% F S=\frac{\sigma}{\text { range }} \cdot 100\right)$.

Table 2.3. The average standard deviation for each loadcell during repeatability testing is compared as a percentage of the loadcell's total range. These results suggest that the precision of the loadcell is a function of its range and style.

\begin{tabular}{ccc}
\hline Style/Range & $\boldsymbol{\sigma}$ & \%FS \\
\hline TAL220/5000 g & $16.3 \mathrm{mg}$ & $0.0004 \%$ \\
TAL221/500 g & $20.7 \mathrm{mg}$ & $0.0041 \%$ \\
TAL221/100 g & $5.0 \mathrm{mg}$ & $0.0050 \%$ \\
\hline
\end{tabular}

The only loadcell tested to its full range was the $100 \mathrm{~g}$ TAL221 because of its smaller range and the results indicate linear behavior of the loadcell across its entire range of measurement. 


\subsubsection{Standard Mass Measurements}

The testing completed with the standard masses yielded results that vary slightly from those returned during comparison testing. The standard deviation of each dataset entirely represents the variation of the measurement output of the scale, without the influence of repeatability or human error when reading the value from the scale. The $5 \mathrm{~kg}$ TAL220 was also tested on two different housings/frames - the 3-D printed PLA housing and a simple wooden frame.

The standard deviations show a similar pattern to the repeatability testing, although both TAL221 load cells performed better in this case than the repeatability testing, while the TAL220 performed slightly worse. The difference in performance may be related to the increased sample size and that the equipment was allowed to warm up prior to testing. These results indicate a direct relationship between load cell range and measurement precision. The standard deviation of each load cell is compared by measurement in Figure 2.13a and overall in Table 2.4. Please note that the $100 \mathrm{~g}$ TAL221 was out of range when measuring the $100 \mathrm{~g}$ standard mass, causing abnormal readout variation and a large error, so that measurement was not included when calculating the average standard deviation shown in Table 2.4.

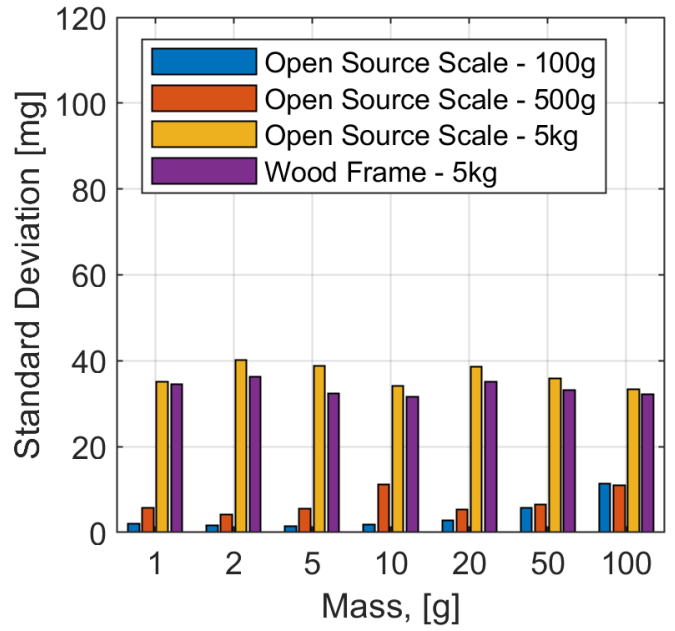

(a)

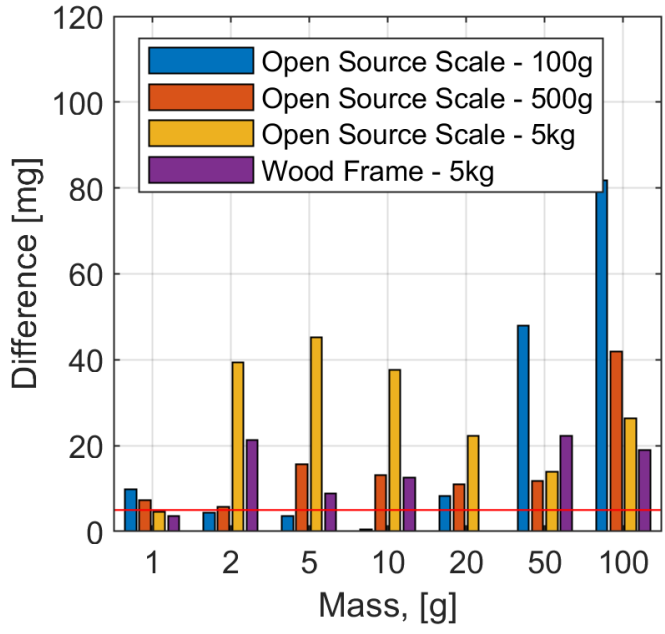

(b)

Figure 2.13. The standard mass test results are shown. (a) The standard deviation (sample of at least 48 measurements) increased with the range of the load cell; there is little difference between the PLA housing and wood frame. (b) The absolute value of the difference between the average measurements of each scale and the nominal mass of the weights is shown. The accuracy range for the weights is indicated by a red line. 
Table 2.4. The average standard deviation for each loadcell during testing with standard masses is compared as a percentage of the loadcell's total range. These results reinforce the idea that the precision of the loadcell is a function of its range and style.

\begin{tabular}{ccc}
\hline Style/Range & $\boldsymbol{\sigma}$ & \%FS \\
\hline TAL220/5000 g & $36.5 \mathrm{mg}$ & $0.0007 \%$ \\
TAL221/500 g & $7.1 \mathrm{mg}$ & $0.0014 \%$ \\
TAL221/100 g & $2.6 \mathrm{mg}$ & $0.0026 \%$ \\
\hline
\end{tabular}

Analyzing the difference between the reported mass and the known mass applied to the scale gives an indication of the accuracy of the scale. Zero-offsets (the scale not reading zero when unloaded) were observed in the $5 \mathrm{~kg}$ TAL220 measurements. This occurred because the scale was zeroed prior to, but not during testing. To correct for the zerooffset, the average reading of the scale immediately prior to each measurement was subtracted from the average measurement. The resulting (corrected) differences between the reported mass and the actual mass are shown in Figure 2.13. This figure also includes a red line indicating the rated accuracy of the weights.

These results indicate that error beyond the accuracy of the weights exist in the scale. This variation may be a result of many factors, including a zero-offset during calibration, temperature variation on the HX711, and, in the case of the $100 \mathrm{~g}$ load cell, overloading.

The $5 \mathrm{~kg}$ TAL220 showed greater variation from the actual mass when installed on the PLA housing when compared to the wooden frame. The behavior is inconsistent and was more pronounced at smaller masses, suggesting that the deformation of the housing is not the cause - although this could very well become an issue with much larger loads. It is possible that the bed of the scale was not seated firmly. Relative motion between the bed and the load cell could change the way the load cell reacts to a load. No significant difference in measurement variation (standard deviation) was observed between the plastic and wooden housings.

\subsubsection{Economic Analysis}

The total mass of the 3-D printed components is $m=18.74 \mathrm{~g}+33.22 \mathrm{~g}+65.44 \mathrm{~g}=$ $117.40 \mathrm{~g}$, bringing the cost of printed components to $\$ 2.46$. Assuming no components are previously owned, the total investment (buying bulk items) for the basic scale is $\$ 51.36$ (\$31.12 if using an Arduino derivative [67]), plus \$14.19 to add an LCD, and \$4.00 to use a wall outlet for power. Accounting for the value of individual components from within bulk purchases, the cost of the scale is $\$ 40.75$ ( $\$ 20.51$ with derivative), plus $\$ 6.26$ for an LCD and $\$ 4.00$ to use a wall outlet for power. These results are summarized in Table 2.5. These estimates do not account for taxes or the cost of shipping, jumper wires, or solder. 
Table 2.5. The cost of the scale varies based on configuration and initial investment. Using a wall outlet for power (as opposed to a computer) costs an extra $\$ 4.00$.

\begin{tabular}{ccc}
\hline Configuration & $\begin{array}{c}\text { Total } \\
\text { Investment }\end{array}$ & $\begin{array}{c}\text { Cost Corrected for } \\
\text { Bulk Purchases }\end{array}$ \\
\hline No LCD & $\$ 51.36 / \$ 31.12^{1}$ & $\$ 40.75 / \$ 20.51$ \\
LCD & $\$ 65.55 / \$ 45.31$ & $\$ 47.01 / \$ 26.77$ \\
\hline
\end{tabular}

${ }^{1}$ Second value uses Arduino derivative

\subsection{Discussion}

\subsubsection{Open Source Scale for Distributed Manufacturing}

The open source scale showed linearity consistent with data sheet claims during testing. The precision of the loadcell is dependent on the type and range of the loadcell, meaning that precision requirements should inform decision making during loadcell selection. All three loadcells tested provide repeatability (one standard deviation) finer than $50 \mathrm{mg}$. A major limitation to the scale is that it is susceptible to environmental effects such as temperature. Documentation from Denver Instruments suggests that powering on the scale for $15+$ minutes prior to testing helps stabilize its output by allowing the components to reach a steady-state temperature [90-91]. This was observed during testing, but not specifically tested. Temperature variations on the HX711 were limited by enclosing the amplifier to reduce airflow, and could be further improved by introducing a temperature sensor to make corrections for environmental variations.

A significant advantage of this scale is that its range and precision is interchangeable to meet varying needs. The serial interface offers the possibility to log data and control the scale during testing, allowing partially and fully automated testing. The open source nature of the scale makes room for improvement and modification where necessary to meet particular needs. Additionally, the modular nature of its design allows for cost savings by removing un-needed components, such as the LCD in the case of automated/computer-based measurement.

The two proprietary, lab-grade scales that were used for comparison testing are out of production. The best estimate for their cost is listings on eBay, which price the APX402 at $\$ 75.00$ and the A-160 at $\$ 425.00[95,96]$. Both lab scales offer RS232 serial interfaces over which the scale can be controlled and interrogated, similar to the open source scale. This means that a scale of comparable (although still lesser) precision to the APX402 can be constructed for as low as one third of the cost of used eBay purchases. New scales with comparable capability to the open source scale come at varying costs, depending on their range, precision, and the presence of a serial interface. Some readily available options are compared to the open scale in Table 2.6. The percent savings $\left[\frac{(\text { os-commercial })}{\text { commercial }}\right]$ achieved by using the open source scale are calculated and summarized. As can be seen by Table 2.6, it is possible to purchase a less expensive scale without serial capability (in this case a kitchen scale), but that there are substantial 
savings using the open source approach for scales with serial capability. For lab-grade scales the open source scale saves between $57 \%$ and $83 \%$ from the commercially available scales.

Table 2.6. Several proprietary scales listed online were compared in price and capability to the open source scale. The comparison indicates the cost savings allowed by the open source scale, which vary greatly depending on the functionality and range desired.

\begin{tabular}{cccc}
\hline Range/Repeatability & $\begin{array}{c}\text { Serial } \\
\text { Capability }\end{array}$ & Retail Cost & $\begin{array}{c}\text { Percent Savings of } \\
\text { Open Source Scale } \\
\text { (\$47.01) }\end{array}$ \\
\hline $500 \mathrm{~g} / 10 \mathrm{mg}[97]$ & No & $\$ 15$ & $\mathrm{NA}$ \\
$500 \mathrm{~g} / 100 \mathrm{mg}[98]$ & No & $\$ 88$ & $-47 \%$ \\
$5000 \mathrm{~g} / 100 \mathrm{mg}[99]$ & No & $\$ 55$ & $-15 \%$ \\
$300 \mathrm{~g} / 1 \mathrm{mg}[100]$ & Yes & $\$ 253$ & $-81 \%$ \\
$500 \mathrm{~g} / 2 \mathrm{mg}[101]$ & Yes & $\$ 115$ & $-59 \%$ \\
$600 \mathrm{~g} / 10 \mathrm{mg}[102]$ & Yes & $\$ 110$ & $-57 \%$ \\
$5000 \mathrm{~g} / 80 \mathrm{mg}[103]$ & Yes & $\$ 289$ & $-83 \%$ \\
\hline
\end{tabular}

In addition, to the benefits of greater control, data-logging, customization, and cost the open source scale has several other advantages. There are often artificial barriers to obtaining scientific equipment in various countries. First, there are often tariffs, duties, "made in \{specific country\}" restrictions, and taxes added to importation of scientific equipment. These can combine with other factors such as cost to limit research, medical care and educational opportunities (particularly in the developing world), which hampers economic development [104-106]. By being able to fabricate a digital scale from lowcost base components, most of which are widely available, scientists, educators, and medical staff can avoid these additional costs and gain access to the tool. Secondly, the most extreme cases, when countries are in desperate need for a wide range of medical/scientific equipment such as currently underway during the COVID-19 pandemic [107], there are even export bans [108] that would limit scientific access to tools. Personal fabrication overcomes such bans. Third, arbitrary definitions of "equipment" rather than "supplies" [109] can limit a scientists' ability to spend money in the way their research demands. Again, being able to build equipment from supplies offers scientists more flexibility to obtain tools even in the face of administrative restrictions.

\subsubsection{Future Technical Work}

Documentation in the A-160 and APX402 manuals suggest that leaving the scale on for 15-60 minutes prior to testing will aid in the accuracy and repeatability of measurements by allowing the components to warm up to a stable temperature. This was not done during some of the documented testing as for the majority of laboratory work such actions are unnecessary and may not be conducive to real-world use cases. This can, however, be tested in future development by logging mass and tracking room and 
component temperatures for long periods of time. Other methods for stabilizing the readout to temperature variations should also be explored. SparkFun's OpenScale microcontroller implements temperature sensing to correct for temperature and this implementation could be quantified [110].

The data-logging/serial interface capability is currently restricted to a scale that is connected to a computer. The scale could be extended to allow automated serial logging using a device such as SparkFun's OpenLog, which stores serial output to an SD memory card [111]. This, in combination with battery power, would result in a portable logging scale for use in a variety of environments.

Testing should be conducted to determine the viability of this scale for use in long-term mass studies. Observations during the documented testing show that the scale tends to drift significantly when first turned on. This drift appears to be largely related to temperature, but may be related to other factors, such as loadcell creep [61], or electrostatic influences from the housing material and surrounding environment. The design could also be tested/modified to work with higher capacity loadcells - the plastic housing is very lightweight, meaning it would deform under heavy loads, resulting in side- and angular-loading on the load cell, which can cause error in measurements [61]. High-weight scales can approach $\$ 1000$ in cost, but this open source scale could be modified to handle larger weight ranges (e.g., with a higher capacity load cell and more rigid housing) without much cost increase $[79,112]$. In addition, because the code is freely shared it can be integrated into more complicated systems such as feedback loop additive manufacturing, melt flow index and dynamic off-gassing experiments. In completing further testing, multiple scales should be constructed to test multiple load cells simultaneously, offering more consistent test conditions and results.

\subsection{Conclusions}

This study provided designs for a low-cost, easily replicable open source lab-grade precision digital scale. The designs are released under open source licenses to enable any lab or individual fabricate it using open source 3-D printers and widely available low-cost components. Over several versions of the design with different load cells the open source scale was found to be repeatable within $50 \mathrm{mg}$, with even better precision depending on the load cell range and style. The scale tracks linearly with proprietary lab-grade scales, meeting the performance specified in the loadcell data sheets, indicating that it is accurate across the range of the loadcell installed. The scale can be produced at significant cost savings compared to scales of comparable range and precision when serial capability is present. The cost savings increase significantly as the range of the scale increases. The ability to use the same hardware and firmware for a variety of loadcells also makes the scale flexible to time-varying needs in a laboratory setting. 


\subsection{References}

1. Fogel, K. Producing Open Source Software: How to Run a Successful Free Software Project; O’Reilly Media Inc.: Sebastopol, CA, USA, 2005; ISBN 9780-596-55299-2.

2. Webert, S. The Success of Open Source; Harvard University Press: Cambridge, MA, USA, 2004; ISBN 978-0-674-01292-9.

3. Gibb, A. Building Open Source Hardware: DIY Manufacturing for Hackers and Makers; Pearson Education: London, UK, 2014.

4. Ackerman, J.R. Toward open source hardware. U. Dayt. L. Rev. 2008, 34, 183222.

5. Pearce, J.M. Building research equipment with free, open-source hardware. Science 2012, 337, 1303-1304.

6. Pearce, J. Open-Source Lab: How to Build Your Own Hardware and Reduce Research Costs; Elsevier; New York, NY, USA, 2013.

7. Da Costa, E.T.; Mora, M.F.; Willis, P.A.; Lago, C.L.; Jiao, H.; Garcia, C.D. Getting started with open-hardware: Development and control of microfluidic devices. Electrophoresis 2014, 35, 2370-2377.

8. Von Hippel, E. Democratizing innovation: The evolving phenomenon of user innovation. JFB 2005, 55, 63-78.

9. Powell, A. Democratizing production through open source knowledge: From open software to open hardware. Media Cult. Soc. 2012, 34, 691-708.

10. Blikstein, P. Digital fabrication and "making" in education: The democratization of invention. FabLabs Mach. Mak. Invent. 2013, 4, 1-21.

11. Gershenfeld, N. Fab: The Coming Revolution on Your Desktop-From Personal Computers to Personal Fabrication; Basic Books: New York, NY, USA, 2008; ISBN 978-0-7867-2204-4.

12. Wittbrodt, B.T.; Glover, A.G.; Laureto, J.; Anzalone, G.C.; Oppliger, D.; Irwin, J.L.; Pearce, J.M. Life-cycle economic analysis of distributed manufacturing with open-source 3-D printers. Mechatronics 2013, 23, 713-726.

13. King, D.L.; Babasola, A.; Rozario, J.; Pearce, J.M. Mobile Open-Source SolarPowered 3-D Printers for Distributed Manufacturing in Off-Grid Communities. Chall. Sustain. 2014, 2, 18-27.

14. Wittbrodt, B.; Laureto, J.; Tymrak, B.; Pearce, J. Distributed manufacturing with 3-d printing: A case study of recreational vehicle solar photovoltaic mounting systems. J. Frugal Innov. 2015, 1, 1-7.

15. Sells, E.; Bailard, S.; Smith, Z.; Bowyer, A.; Olliver, V. RepRap: The replicating rapid prototyper-maximizing customizability by breeding the means of production. In Proceedings of the World Conference on Mass Customization and Personalization, Cambridge, MA, USA, 7-10 October 2007.

16. Jones, R.; Haufe, P.; Sells, E.; Iravani, P.; Olliver, V.; Palmer, C.; Bowyer, A. RepRap-the replicating rapid prototyper. Robotica 2011, 29, 177-191.

17. Bowyer, A. 3D Printing and humanity's first imperfect replicator. 3D Print. Addit. Manuf. 2014, 1, 4-5. 
18. Kietzmann, J.; Pitt, L.; Berthon, P. Disruptions, decisions, and destinations: Enter the age of 3-D printing and additive manufacturing. Bus. Horiz. 2015, 58, 209-215.

19. Lipson, H.; Kurman, M. Fabricated: The New World of 3D Printing; John Wiley \& Sons: Chichester, UK, 2013.

20. Gwamuri, J.; Wittbrodt, B.; Anzalone, N.; Pearce, J. Reversing the trend of large scale and centralization in manufacturing: The case of distributed manufacturing of customizable 3-d-printable self-adjustable glasses. Chall. Sustain. 2014, 2, 30-40.

21. Attaran, M. The rise of 3-D printing: The advantages of additive manufacturing over traditional manufacturing. Bus. Horiz. 2017, 60, 677-688.

22. Tanikella, N.G.; Wittbrodt, B.; Pearce, J.M. Tensile strength of commercial polymer materials for fused filament fabrication 3D printing. Addit. Manuf. 2017, 15, 40-47.

23. Baden, T.; Chagas, A.M.; Gage, G.; Marzullo, T.; Prieto-Godino, L.L.; Euler, T. Open labware: 3-D printing your own lab equipment. PLoS Biol. 2015, 13, e1002086, doi:10.1371/journal.pbio.1002086.

24. Coakley, M.; Hurt, D.E. 3D Printing in the laboratory: Maximize time and funds with customized and open-source labware. J. Lab. Autom. 2016, 21, 489-495.

25. Zhang, C.; Wijnen, B.; Pearce, J.M. Open-Source 3-D platform for low-cost scientific instrument ecosystem. J. Lab. Autom. 2016, 21, 517-525.

26. Dhankani, K.C.; Pearce, J.M. Open source laboratory sample rotator mixer and shaker. HardwareX 2017, 1, 1-12.

27. Trivedi, D.K.; Pearce, J.M. Open Source 3-D Printed Nutating Mixer. Appl. Sci. 2017, 7, 942.

28. Zhang, C.; Anzalone, N.C.; Faria, R.P.; Pearce, J.M. Open-Source 3D-Printable Optics Equipment. PLoS ONE 2013, 8, e59840.

29. Winters, B.J.; Shepler, D. 3D printable optomechanical cage system with enclosure. Hardware X 2018, 3, 62-81.

30. Agcayazi, T.; Foster, M.; Kausche, H.; Gordon, M.; Bozkurt, A. Multi-axis stress sensor characterization and testing platform. HardwareX 2019, 5, e00048.

31. Delmans, M.; Haseloff, J. $\mu$ Cube: A Framework for $3 D$ Printable Optomechanics; Ubiquity Press: London, UK, 2018.

32. Sharkey, J.P.; Foo, D.C.W.; Kabla, A.; Baumberg, J.J.; Bowman, R.W. A onepiece 3D printed flexure translation stage for open-source microscopy. Rev. Sci. Instrum. 2016, 87, 025104.

33. Collins, J.T.; Knapper, J.; Stirling, J.; Mduda, J.; Mkindi, C.; Mayagaya, V.; Mwakajinga, G.A.; Nyakyi, P.T.; Sanga, V.L.; Carbery, D.; et al. Robotic microscopy for everyone: The OpenFlexure Microscope. bioRxiv 2019, 11, $2447-2460$.

34. Grant, S.D.; Cairns, G.S.; Wistuba, J.; Patton, B.R. Adapting the 3D-printed Openflexure microscope enables computational super-resolution imaging. F1000Res 2019, 8, doi:10.12688/f1000research.21294.1

35. Anzalone, G.C.; Glover, A.G.; Pearce, J.M. Open-Source Colorimeter. Sensors 2013, 13, 5338-5346. 
36. Kelley, C.D.; Krolick, A.; Brunner, L.; Burklund, A.; Kahn, D.; Ball, W.P.; Weber-Shirk, M. An Affordable Open-Source Turbidimeter. Sensors 2014, 14, 7142-7155.

37. Wijnen, B.; Anzalone, G.C.; Pearce, J.M. Open-source mobile water quality testing platform. J. Water Sanit. Hyg. Dev. 2014, 4, 532-537.

38. Wittbrodt, B.T.; Squires, D.A.; Walbeck, J.; Campbell, E.; Campbell, W.H.; Pearce, J.M. Open-Source Photometric System for Enzymatic Nitrate Quantification. PLoS ONE 2015, 10, e0134989.

39. Wijnen, B.; Hunt, E.J.; Anzalone, G.C.; Pearce, J.M. Open-Source Syringe Pump Library. PLoS ONE 2014, 9, e107216.

40. Bravo-Martinez, J. Open source 3D-printed $1000 \mu \mathrm{L}$ micropump. HardwareX 2018, 3, 110-116.

41. Pusch, K.; Hinton, T.J.; Feinberg, A.W. Large volume syringe pump extruder for desktop 3D printers. HardwareX 2018, 3, 49-61.

42. Garcia, V.E.; Liu, J.; DeRisi, J.L. Low-cost touchscreen driven programmable dual syringe pump for life science applications. HardwareX 2018, 4, e00027.

43. Klar, V.; Pearce, J.M.; Kärki, P.; Kuosmanen, P. Ystruder: Open source multifunction extruder with sensing and monitoring capabilities. HardwareX 2019, 6, e00080.

44. Pearce, J.M.; Anzalone, N.C.; Heldt, C.L. Open-Source Wax RepRap 3-D Printer for Rapid Prototyping Paper-Based Microfluidics. J. Lab. Autom. 2016, 21, 510-516.

45. Lake, J.R.; Heyde, K.C.; Ruder, W.C. Low-cost feedback-controlled syringe pressure pumps for microfluidics applications. PLOS ONE 2017, 12, doi:10.1371/journal.pone.0175089.

46. Kong, D.S.; Thorsen, T.A.; Babb, J.; Wick, S.T.; Gam, J.J.; Weiss, R.; Carr, P.A. Open-source, community-driven microfluidics with Metafluidics. Nat. Biotechnol. 2017, 35, 523-529.

47. Niezen, G.; Eslambolchilar, P.; Thimbleby, H. Open-source hardware for medical devices. BMJ Innov. 2016, 2, 78-83.

48. Daniel, K.F.; Peter J, G. Open-Source Hardware Is a Low-Cost Alternative for Scientific Instrumentation and Research. Mod. Instrum. 2012, 2012, doi:10.4236/mi.2012.12002.

49. Pearce, J.M. Laboratory equipment: Cut costs with open-source hardware. Nature 2014, 505, doi: 10.1038/505618d.

50. Pearce, J.M. Impacts of Open Source Hardware in Science and Engineering. Bridge 2017, 47, 24-31.

51. Pearce, J. Quantifying the Value of Open Source Hardware Development. Mod. Econ. 2015, 6, 1-11.

52. Pearce, J.M. Return on investment for open source scientific hardware development. Sci. Public Policy 2016, 43, 192-195.

53. Hietanen, I.; Heikkinen, I.T.S.; Savin, H.; Pearce, J.M. Approaches to open source 3-D printable probe positioners and micromanipulators for probe stations. HardwareX 2018, 4, e00042. 
54. Dryden, M.D.M.; Fobel, R.; Fobel, C.; Wheeler, A.R. Upon the Shoulders of Giants: Open-Source Hardware and Software in Analytical Chemistry. Anal. Chem. 2017, 89, 4330-4338.

55. Quartz Crystal Microbalance with Dissipation Monitoring: The First Scientific QCM Entirely Open Source. Available online: https://openqcm.com/ (accessed on 7 April 2020).

56. Politi, J.; Dardano, P.; Caliò, A.; Iodice, M.; Rea, I.; De Stefano, L. Reversible sensing of heavy metal ions using lysine modified oligopeptides on porous silicon and gold. Sens. Actuators B Chem. 2017, 244, 142-150.

57. Frijns, E.; Verstraelen, S.; Stoehr, L.C.; Van Laer, J.; Jacobs, A.; Peters, J.; Tirez, K.; Boyles, M.S.P.; Geppert, M.; Madl, P.; et al. A Novel Exposure System Termed NAVETTA for In Vitro Laminar Flow Electrodeposition of Nanoaerosol and Evaluation of Immune Effects in Human Lung Reporter Cells. Environ. Sci. Technol. 2017, 51, 5259-5269.

58. Ventura, B.D.; Iannaccone, M.; Funari, R.; Ciamarra, M.P.; Altucci, C.; Capparelli, R.; Roperto, S.; Velotta, R. Effective antibodies immobilization and functionalized nanoparticles in a quartz-crystal microbalance-based immunosensor for the detection of parathion. PLOS ONE 2017, 12, e0171754.

59. Fries, M.D.F. The Opera Instrument: An Advanced Curation Development for Mars Sample Return Organic Contamination Monitoring; NASA: Woodlands, TX, USA, 2018.

60. Muckley, E.S.; Naguib, M.; Ivanov, I.N. Multi-modal, ultrasensitive, widerange humidity sensing with Ti3C2 film. Nanoscale 2018, 10, 21689-21695.

61. Scale Manufacturers Association Load Cell Application and Test Guideline. Available online:

http://www.scalemanufacturers.org/pdf/loadcellapplicationtestguidelineapril201 $\underline{0 . p d f}$ (accessed on 13 April 2020).

62. Xiamen Ocular 1602 LCD Data Sheet. Available online: https://www.sparkfun.com/datasheets/LCD/GDM1602K.pdf (accessed on 30 March 2020).

63. AVIA Semiconductor HX711 Data Sheet. Available online: https://cdn.sparkfun.com/datasheets/Sensors/ForceFlex/hx711_english.pdf (accessed on 30 March 2020).

64. Arduino Arduino-Board. Available online: https://www.arduino.cc/en/reference/board (accessed on 30 March 2020).

65. Scale Manufacturers Association SMA SCP-0499 Scale Serial Communication Protocol 1999. Available online: http://www.scalemanufacturers.org/PDF/ScaleCommProtocol5199M1.pdf (accessed on 13 April 2020).

66. Arduino Nano. Available online: https://store.arduino.cc/usa/arduino-nano (accessed on 6 April 2020).

67. Geekcreit Nano V3 Module with USB Cable. Available online: https://www.banggood.com/ATmega328P-Nano-V3-Module-ImprovedVersion-With-USB-Cable-Development-Board-p-933647.html (accessed on 6 April 2020). 
68. AmazonBasics USB A-miniB. Available online:

https://www.amazon.com/AmazonBasics-USB-2-0-Cable-

Male/dp/B00NH13S44 (accessed on 6 April 2020).

69. Amazon 5V USB Wall Charger. Available online:

https://www.amazon.com/Certified-Charger-FONKEN-universal-

Compatible/dp/B07DCR29GN/ref=sr 1 3? crid=2T9NB566GJAS4\&dchild=1\&

keywords $=5 \mathrm{v}+\mathrm{usb}+$ power + supply\&qid $=1586183620 \& \mathrm{~s}=$ electronics \&sprefix $=5$ $\mathrm{v}+\mathrm{usb}+\mathrm{p} \% 2 \mathrm{Celectronics} \% 2 \mathrm{C} 213 \& \mathrm{sr}=1-3$ (accessed on 6 April 2020).

70. Amazon HX711 and TAL220. Available online:

https://www.amazon.com/KNACRO-Converter-Breakout-PortableElectronic/dp/B078W2VCP2/ref=sr 1 1 ?dchild=1\&keywords=tal220\&qid $=15$

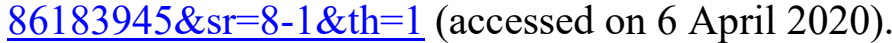

71. Industries, Adafruit. Tactile Button Switch $(6 \mathrm{~mm}) \times 20$ pack. Available online: https://www.adafruit.com/product/367 (accessed on 6 April 2020).

72. Industries, Adafruit. Adafruit Full Sized Breadboard. Available online:

https://www.adafruit.com/product/239 (accessed on 6 April 2020).

73. Banggood 40pcs Double Side Prototype PCB. Available online: https://www.banggood.com/Geekcreit-40pcs-FR-4-2 54mm-Double-SidePrototype-PCB-Printed-Circuit-Board-p-995732.html (accessed on 6 April 2020).

74. Arducam $160216 \times 2$ LCD Display Module. Available online: https://www.amazon.com/Arducam-Display-Controller-CharacterBacklight/dp/B019D9TYMI (accessed on 6 April 2020).

75. Potentiometers|Mouser. Available online: https://www.mouser.com/PassiveComponents/Potentiometers-Trimmers-Rheostats/Potentiometers/ /N9q0yp?Ns=Pricing $\mid 0$ (accessed on 14 April 2020).

76. Resistor Kit - 1/4W (500 total) - COM-10969-SparkFun Electronics. Available online: https://www.sparkfun.com/products/10969 (accessed on 6 April 2020).

77. Headers, Adafruit. Available online: https://www.adafruit.com/category/154 (accessed on 6 April 2020).

78. HTC-Sensor TAL220 Data Sheet. Available online: https://cdn.sparkfun.com/datasheets/Sensors/ForceFlex/TAL220M4M5Update.p df (accessed on 4 April 2020).

79. HTC-Sensor TAL221 Data Sheet. Available online: https://cdn.sparkfun.com/assets/9/9/a/f/3/TAL221.pdf (accessed on 4 April 2020).

80. Mini Load Cell_-100g, Straight Bar (TAL221), SparkFun Electronics. Available online: https://www.sparkfun.com/products/14727 (accessed on 6 April 2020).

81. Open Source Laboratory Logging Digital Scale. Available online: https://osf.io/me9a8/ DOI 10.17605/OSF.IO/ME9A8.

82. GNU General Public License Version 3. Available online: https://www.gnu.org/licenses/gpl-3.0.en.html (accessed on 29 March 2019). 
83. Cura LulzBot Edition. Available online: https://www.lulzbot.com/cura (accessed on 2 April 2019).

84. LulzBot LulzBot TAZ 6. Available online: https://www.lulzbot.com/store/printers/lulzbot-taz-6 (accessed on 20 April 2020).

85. Fritzing. Available online: http://fritzing.org/ (accessed on 28 March 2020).

86. Arduino HelloWorld. Available online: https://www.arduino.cc/en/Tutorial/HelloWorld (accessed on 28 March 2020).

87. SparkFun Basic Character LCD Hookup Guide-learn.sparkfun.com. Available online: https://learn.sparkfun.com/tutorials/basic-character-lcd-hookup-guide (accessed on 30 March 2020).

88. Bogde $H X 711$. Available online: https://github.com/bogde/HX711 (accessed on 13 April 2020).

89. Hans-Christoph Steiner LiquidCrystal; Arduino Libraries. Available online: https://github.com/arduino-libraries/LiquidCrystal (accessed on 13 April 2020).

90. Denver Instruments APX-402 Operators Manual. Available online: http://www.denverinstrument.com/denverusa/media/pdf/archive manuals/OpMan-Apex-RevC.pdf (accessed on 16 April 2019).

91. Denver Instruments A-160 Operators Manual. Available online: http://www.denverinstrument.com/denverusa/media/pdf/archive manuals/OpM an_A-Series.pdf (accessed on 16 April 2019).

92. Sule, S.S.; Petsiuk, A.L.; Pearce, J.M. Open Source Completely 3-D Printable Centrifuge. Instruments 2019, 3, 30.

93. EIA. Electric Power Monthly. Available online: https://www.eia.gov/electricity/monthly/epm table_grapher.php?t=epmt 5 _ 6 a (accessed on 16 April 2019).

94. White MH Build Series PLA Filament $-2.85 \mathrm{~mm}(1 \mathrm{~kg})$. Available online: https://www.matterhackers.com/store/3d-printer-filament/300mm-pla-filamentwhite-1-kg (accessed on 20 April 2020).

95. eBay Denver APX 402 Scale for sale online. Available online: https://www.ebay.com/c/669060320 (accessed on 14 April 2020).

96. eBay Denver A-160 Digital Scale for Sale Online. Available online: https://www.ebay.com/itm/Denver-Instruments-Company-Digital-Scale-A-160/161055820362 (accessed on 14 April 2020).

97. AMIR Digital Kitchen Scale, 500g-0.01g. Available online: https://www.amazon.com/AMIR-Upgraded-500g-0-01g-StainlessBatteries/dp/B01HCKQG7G (accessed on 22 April 2020).

98. Lab Analytical Balance Scale, $500 \mathrm{~g} \times 0.01 \mathrm{~g}$ High Precision Electronic Scientific Scale Peeling Weight Digital Kitchen Precision Balance Jewelry Gold Scale with $200 \mathrm{~g}$ Weight Self-Correcting Function (US): Industrial \& Scientific. Available online: https://www.amazon.com/Analytical-500gx0-01g-ElectronicScientific-Self-Correcting/dp/B08591CKQT/ (accessed on 23 April 2020).

99. Mein LAY Lab Analytical Electronic Balance Scale Laboratory Balance Digital Kitchen Balance Scale Jewelry Gold Scale Precision Balance (0.01/2000g): Home \& Kitchen. Available online: https:/www.amazon.com/Mein-LAY- 
Analytical-Electronic-Laboratory/dp/B07VRCSFBY/ (accessed on 23 April 2020).

100. U.S. SOLID $0.001 \mathrm{~g} 1 \mathrm{mg}$ Analytical Digital Lab Precision Balance Scale 300g. Available online: https://www.amazon.com/U-S-Analytical-Digital-PrecisionBalance/dp/B017ADAA7C/ (accessed on 23 April 2020).

101. U.S. Solid 0.001g 1mg Digital Analytical Balance w/ RS232 Interface. Available online: https://www.amazon.com/U-S-Solid-Analytical-LaboratoriesCalibration/dp/B07Y2SXMTC (accessed on 22 April 2020).

102. U.S. Solid Precision Lab Scale $1000 \mathrm{~g} \times 0.01 \mathrm{~g}$-High Precision Analytical Balance w/ RS232 Interface, Detachable Draft Shield, Calibration Weight, 100240 VAC. Available online: https://www.amazon.com/U-S-Solid-PrecisionAnalytical-Detachable/dp/B07YL1B146/?th=1 (accessed on 23 April 2020).

103. CGOLDENWALL 5000g 5kg 0.01g Lab Analytical Balance RS232. Available online: https://www.amazon.com/Analytical-Precision-Electronic-CalibrationCommercial/dp/B07DCM3CFC (accessed on 22 April 2020).

104. Ross, A.R.; Lewin, K.M. Science Kits in Developing Countries: An Appraisal of Potential; UNESCO: London, UK, 1992; pp. 1-120.

105. Gwamuri, J.; Pearce, J.M. Open source 3D printers: An appropriate technology for building low cost optics labs for the developing communities. In Proceedings of the 14th Conference on Education and Training in Optics and Photonics: ETOP 2017, Hangzhou, China, 29-31 May 2017; Volume 10452, p. $104522 \mathrm{~S}$.

106. De Maria, C.; Di Pietro, L.; Ravizza, A.; Lantada, A.D.; Ahluwalia, A.D. Chapter 2-Open-source medical devices: Healthcare solutions for low-, middle-, and high-resource settings. In Clinical Engineering Handbook, 2nd ed.; Iadanza, E., Ed.; Academic Press: Cambridge, MA, USA, 2020; pp. 7-14, ISBN 978-0-12-813467-2.

107. Pearce, J. Distributed Manufacturing of Open-Source Medical Hardware for Pandemics. Preprints 2020, 4, 49, doi:10.20944/preprints202004.0054.v1.

108. Sebastian, C.; di Donato, V. CNN. In the Race to Secure Medical Supplies, Countries Ban or Restrict Exports. Available online: https://www.cnn.com/2020/03/27/business/medical-supplies-exportban/index.html (accessed on 15 April 2020).

109. Pearce, J.M. Undermined by overhead accounting. Science 2016, 352, 158-159.

110. SparkFun OpenScale Applications and Hookup Guide-SparkFun. Available online: https://learn.sparkfun.com/tutorials/openscale-applications-and-hookupguide\#calibration-suggestions (accessed on 16 March 2020).

111. SparkFun OpenLog-DEV-13712. Available online: https://www.sparkfun.com/products/13712 (accessed on 14 April 2020).

112. GMM Technoworld $50 \mathrm{~kg} / 1 \mathrm{~g} 2 \mathrm{~kg} / 0.05 \mathrm{~g}$ Digital Counting Scale with Dual Platter \& PC Connection. Available online: https://testmeter.sg/webshaper/store/viewprod.asp?pkproductitem=432\&pkprod uct $=$ (accessed on 22 April 2020). 


\section{Open Source Vacuum Oven for Low-Temperature Drying}

Vacuum drying can dehydrate materials further than dry heat methods while protecting sensitive materials from thermal degradation. Many industries have shifted to vacuum drying as cost- or time-saving measures. Small-scale vacuum drying, however, has been limited by high costs of specialty scientific tools. To make vacuum drying more accessible this study provides design and performance information for a small-scale open source vacuum oven, which can be fabricated from off-the-shelf and 3-D printed components. The oven is tested for drying speed and effectiveness on both waste plastic polyethylene terephthalate (PET) and a consortium of bacteria developed for bioprocessing of terephthalate wastes to assist in distributed recycling of PET for both additive manufacturing as well as potential food. Both of these materials can be damaged when exposed to high temperatures, making vacuum drying a desirable solution. The results showed the open source vacuum oven was effective at drying both plastic and biomaterials, drying at a higher rate than a hot-air dryer for small samples or for low volumes of water. The system can be constructed for less than $20 \%$ of the cost of commercial vacuum dryers for several laboratory-scale applications including dehydration of bio-organisms, drying plastic for DRAM, and chemical processing.

\subsection{Introduction}

Dehydration, also referred to as drying or dewatering, is the process of removing water from a system or material [1]. It is an important step in materials processes spanning several industries. Many polymers experience chemical breakdown called hydrolysis when exposed to water, particularly at high temperatures (i.e. during manufacturing) [2], making water removal incredibly important in the plastics industry. This is an important step for both industrial scale manufacturing [3-5] as well as distributed recycling and additive manufacturing (DRAM) [6-8] particularly of recycled polyethylene terephthalate (rPET), which is the most commonly used plastic [9]. Additionally, paper is manufactured by suspending pulp in water, then carefully removing that water in order to form paper of desired parameters [10]. The bioenergy industry dries biomass fuels prior to burning them in boilers to increase efficiency [11]. Woodworkers remove as much moisture as possible from pieces of soft wood before stabilizing them, a process which hardens otherwise unworkable wood so it can be crafted into fine pieces of art [12]. Microbiologists desiccate microorganisms in order to halt reproduction without necessarily killing them [13]. Dehydration is also commonly used for food preservation [14].

Given the widespread demand for processes to remove water from materials, many drying methods have been developed and refined. The basic mechanism for removing water from a material is to reduce the energy required for the water to leave the material $[1,15]$. This can be accomplished by increasing the moisture capacity of the air, either by heating the air or removing existing moisture with a sacrificial desiccant material; or by reducing the saturation temperature (boiling point) of water by introducing the material to vacuum [16]. Vacuum processing allows dehydration at low temperatures, which is most 
valuable for processing heat-sensitive materials such as bio-organisms, food, and thermopolymers that degrade at high temperatures [17]. When combined with elevated temperatures, vacuum drying can dehydrate materials at an increased rate compared with dry heat methods $[16,18]$. This has encouraged a shift in recent years for multiple industries to move away from dry heat-based dehydration to vacuum drying as a cost- or time-saving measure $[10,16]$.

Small scale drying technologies are much more limited. Consumer products for drying materials such as food, wood, or plastic (i.e., for 3-D printing) are limited to hot-air dryers, such as food dehydrators, toaster ovens, or typical convection ovens [12,19,20], or passive desiccant solutions that a consumer can assemble with bulk silica gel and a sealed container [21,22]. More advanced solutions, such as freeze dryers and vacuum ovens, are technically available to consumers, but at extreme costs on the order of thousands of dollars $[23,24]$. Vacuum ovens are used by the plastics industry, drying high volumes of plastic in as little as one hour [25].

In an effort to expand the available low-cost and accessible drying solutions, this study provides design and performance information for a small-scale open source vacuum oven. A vacuum oven puts the material being dried under a vacuum at elevated (but still relatively low) temperature in order to dry out the material at a high rate. The novel vacuum oven presented in this study uses an off-the-shelf vacuum chamber, augmented with a digitally-replicable compressed air-powered vacuum pump, and a simple thermal control system based on the open source Arduino microcontroller platform for heating samples in the oven. The oven is tested for drying speed and effectiveness on rPET plastic for DRAM and on a consortium of single cell microorganisms used for biological conversion of waste plastic chemical components to food. Both can be damaged when exposed to high temperatures, making vacuum drying a desirable solution. During these tests, the performance of the vacuum oven was compared to an off-the-shelf food dehydrator and a control. The results are discussed in the context of both polymer and biomaterial dewatering as well as recommendations for future work.

\subsection{Materials and Methods}

\subsubsection{Design}

The vacuum oven constructed in this study has two primary components: 1) a vacuum chamber and 2) a heating element. The vacuum chamber and heating element together serve to form the vacuum oven. System performance was monitored with an open source high-precision mass balance [26] and multimeters.

\subsubsection{Vacuum System}

The physical vacuum chamber selected is an off-the-shelf vacuum chamber, which is designed to handle vacuum pressures up to $29 \mathrm{inHg}$ [27]. This is a sufficiently deep vacuum to allow drying at or only slightly above room temperature [28]. Vacuum pressure (the difference between evacuated pressure and atmospheric pressure) is a convenient metric for discussing mechanical behavior of the chamber and vacuum pump, 
as their performance is directly linked to vacuum pressure - in how much pressure the chamber can withstand, and how deep a vacuum the pump can draw. However, the thermodynamic behavior of water depends on the absolute pressure of the atmosphere the water is exposed to. Since atmospheric pressure varies with elevation and weather, the vacuum pressure required to reach a certain absolute pressure can vary. This can be confusing when looking at the performance of a vacuum oven. To help distinguish between these two quantities, they will be expressed in different units following the protocol for water saturation tables [29]. Vacuum pressure will be noted in inHg, where 0 inHg is atmospheric pressure at sea level, and $29.9 \mathrm{inHg}$ is absolute vacuum. Absolute pressure will be noted in $\mathrm{kPa}$, where $0 \mathrm{kPa}$ is absolute vacuum, and $101.3 \mathrm{kPa}$ is atmospheric pressure at sea level. Finally, gauge pressure, pressure measured above atmospheric pressure, will be expressed in psig (pounds-per-square-inch-gauge). Each set of units was selected directly from the instrument or reference material providing the relevant measurement. The vacuum gauge provides inHg [30], thermodynamic tables list saturation points of water with absolute pressure in $\mathrm{kPa}$ [28], and the air compressor measures output pressure in psig [31].

An air ejector was selected to act as the vacuum pump for this system and details of the selection process are provided in Appendix A. Air ejectors (also called venturi ejectors, venturi vacuums, air-powered vacuum pumps, and air-jet ejectors) use high-pressure air and a combination of converging-diverging nozzles to create a vacuum pressure [32-34]. The shape and size of the nozzles can be varied to affect the required inlet pressure, the rate of evacuation, the depth of vacuum, and the volume of air consumed by the ejector $[33,35]$. The practical limit of a single-stage air ejector is around $28 \mathrm{inHg}$ vacuum, which translates to an absolute pressure of around $6.6 \mathrm{kPa}$ [34] and thus brings the saturation temperature of water just below $40 \circ \mathrm{C}$ [28]. Other methods can achieve a deeper vacuum, but this allows for a sufficiently low temperature to encourage rapid evaporation. In addition, an air ejector has the advantage of being driven by compressed air. Since air is being forced through the ejector and out into the atmosphere, never moving through a machine, there is no risk of damage to equipment due to water or other materials which may get caught up in the flow of evacuated air [36]. On an industrial scale, compressed air is often already present for other processes, mitigating the cost of introducing a vacuum system [36]. Similarly, in small labs or distributed fablabs and makerspaces, an air compressor is much more likely to be a part of the existing toolset (and more generally useful) than a mechanical vacuum pump. Air ejectors are commercially available at a similar cost to vacuum pumps [37]. Due to the robustness and cost effectiveness of an air-ejector, this was selected to evacuate the chamber.

\subsubsection{Thermal Controls}

The thermal control system is composed of a heater, controlled by a relay and a microcontroller. The micro-controller measures the temperature of the heater pad with a negative-temperature-coefficient (NTC) thermistor. A thermistor is a sensor whose resistance varies as a function of temperature. They are commonly used in 3-D printing and offer measurement precision on the order of $0.2{ }^{\circ} \mathrm{C}$, though the accuracy depends heavily on the accuracy of the model used to calculate temperature, the temperature range 
being measured, and the resolution of the analog-to-digital converter (ADC) used to measure the thermistor $[38,39]$. The resistance of the thermistor is measured by an open source Arduino microcontroller [40] using a voltage divider with a known reference voltage and resistance. The measured resistance can be used to estimate the temperature (and vice versa) using what is known as the Steinhart-Hart equation [41], which characterizes a thermistor based on three resistance values, measured at three known temperatures. This model tends to be accurate within half a degree Celsius over the calibration range [41] but is often simplified to a two-point model which requires a single coefficient, the Beta coefficient, and is simpler to calibrate and compute while measuring. This simplified model maintains similar accuracy over a smaller temperature range $[39,42,43]$. The simplified Steinhart-Hart equation was used for measuring the temperature of the heating element.

The challenge of building a robust thermal control system for a vacuum oven is significant, particularly with the use of off-the-shelf parts. The primary mode of heat transfer inside the vacuum chamber is conduction, which cannot be accurately modeled for the design of a controller, as it depends on many factors including the type, volume, density, and surface area of the material inside, as well as the rate of heat loss to the environment. In order to control temperature inside the chamber without damaging the chamber, the approach selected was to track the temperature of the heating element $-\mathrm{a}$ flexible silicone heating element, used as engine block heaters or 3-D printer bed heaters. These offer a large surface area and relatively low power per unit surface area (Watt density), which is preferable for low set temperatures, since it reduces the rate at which the heater must switch on and off to maintain a set temperature [44]. The controller operates on the assumption that sufficient insulation and time allow the inside of the vacuum chamber to reach equilibrium at or near the temperature of the heating element. In order to quantify the inevitable temperature difference between the heater and the inside of the chamber, the gradient was measured with loosely packed rPET in the chamber. These measurements were used to inform set temperatures for the oven during testing that manually correct for the temperature difference. It was found, from the perspective of drying speed, that this approach is applicable on small samples (tens of grams). In order to control the temperature of the heater, a simple relay control system was used. This was selected because the thick metal of the chamber sufficiently filters out the switching effects of the relay, providing a smooth temperature curve inside the chamber.

\subsubsection{Bill of Materials}

The vacuum chamber and thermal control system equipment are separate, fairly interchangeable components. As such, they are provided in two sections of the Bill of Materials in Table 3.1. A full bill of materials (BOM), including unit cost (corrected for the amount of a bulk material actually consumed), links for purchase, and additional notes, is available in a repository on the Open-Science Framework (OSF) [45]. 
Table 3.1. Visual bill of materials separated between the two primary components.

\begin{tabular}{|c|c|}
\hline Component & Photograph \\
\hline \multicolumn{2}{|c|}{ Vacuum Chamber } \\
\hline \multicolumn{2}{|l|}{$\begin{array}{l}\text { Air Compressor } \\
\quad \$ 99[31]\end{array}$} \\
\hline \multicolumn{2}{|l|}{$\begin{array}{l}\text { Fixed-Flow Air-Powered Vacuum Pump } \\
\qquad \$ 79.75[37]\end{array}$} \\
\hline \multicolumn{2}{|l|}{$\begin{array}{l}\text { Vacuum Chamber, including: } \\
\text { Lid with hole for plumbing } \\
\text { M10 Nut } \\
\text { M10 Washers (nylon or rubber) } \\
\$ 89.99[27]\end{array}$} \\
\hline \multicolumn{2}{|l|}{$\begin{array}{l}\text { 10sq ft Reflectix Double Sided Insulation } \\
\qquad 13.57 / 33.3 \mathrm{sq} \mathrm{ft}[46]\end{array}$} \\
\hline $\begin{array}{l}\text { Vacuum Gauge with Rubber Hose } \\
\qquad \$ 24.99[30]\end{array}$ & \\
\hline
\end{tabular}


1ft High Temperature Flue Tape $\$ 8.82 / 15 \mathrm{ft}$ [47]

3-D Printed Vacuum Pump Intake Connector [45]

Printed in shown orientation, threads up.

3-D Printed Vacuum Pump Vacuum Connector [45]

Printed in shown orientation, fine threads up

\section{Thermal Control System}

120V Silicone Heating Pad, $1.16 \mathrm{~W} / \mathrm{cm}^{2}$

$\left(7.5 \mathrm{~W} / \mathrm{in}^{2}\right)$

$19.99[48]$

Solid State Relay (5V Input, 120VAC 2A+ output) $\$ 25.44$ [49] 


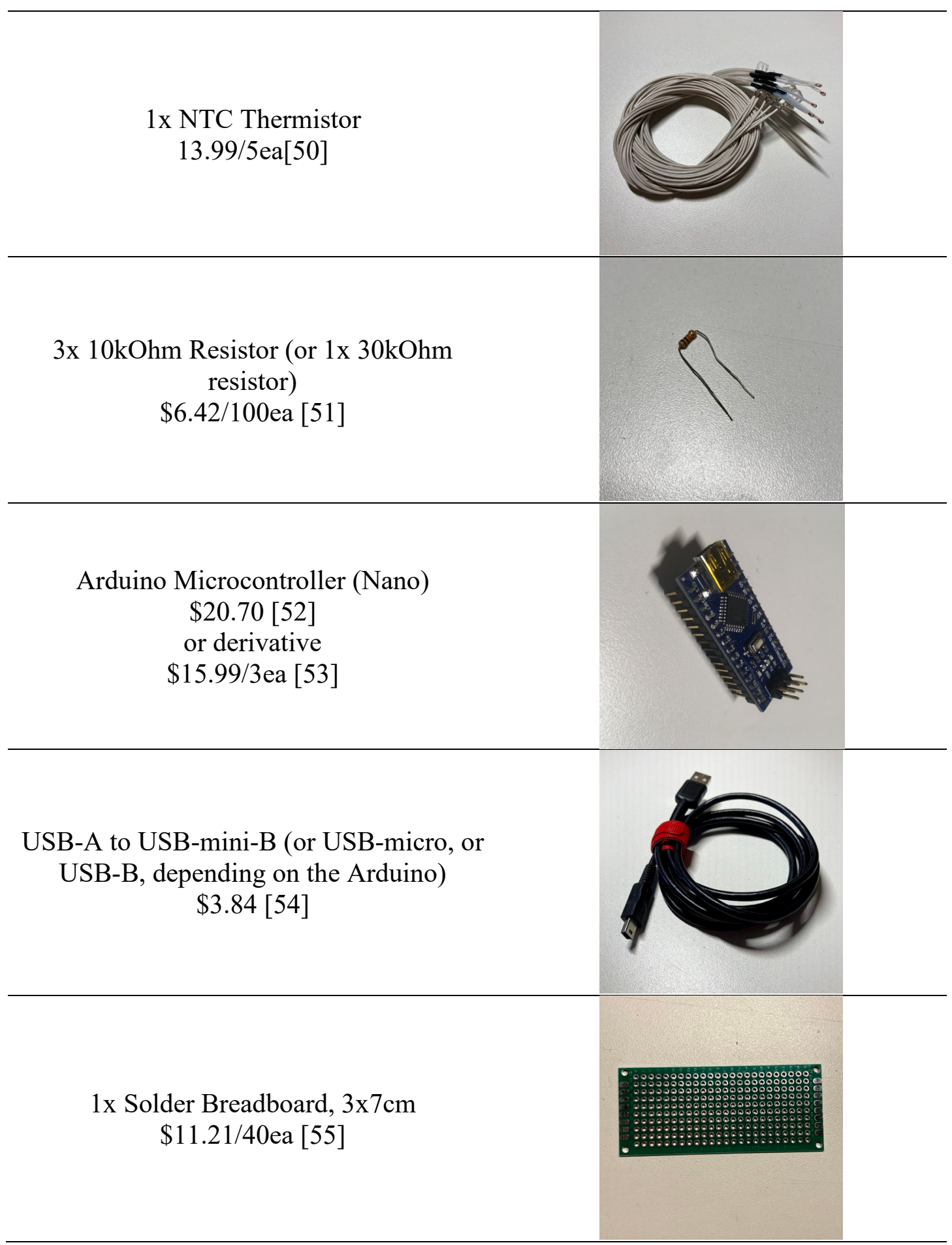




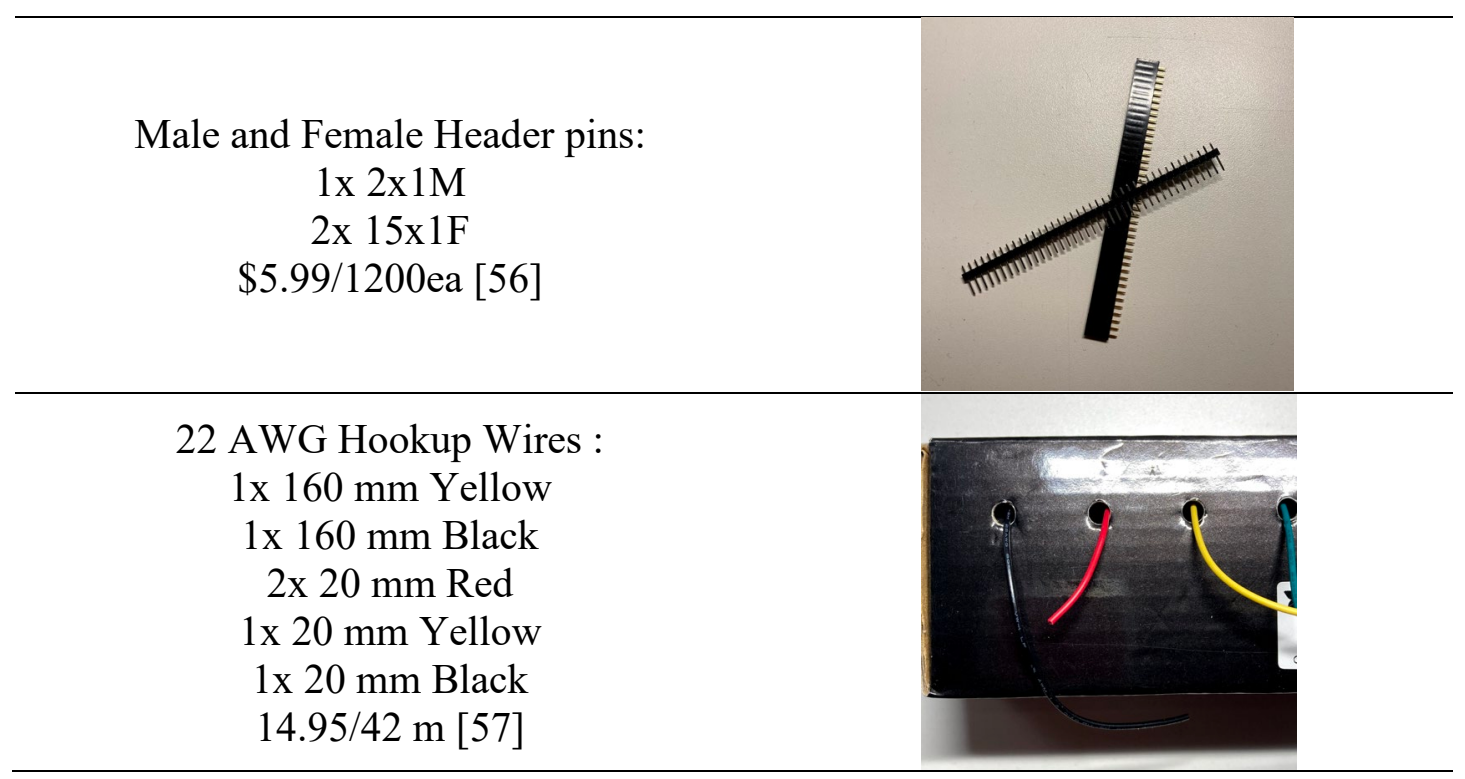

\subsubsection{Manufacturing and Assembly}

\subsubsection{Vacuum Chamber}

To prepare the vacuum chamber for assembly, two strips of Reflectix insulation were cut about $5 \mathrm{~cm}$ wide and 2-3 times the circumference of the chamber in length. These were used to build an air gap between the Reflectix and the sides of the chamber, per Reflectix's installation recommendations [58]. They also helped the bulk insulation span over handles and clasps protruding from the wall of the chamber. Another piece of Reflectix insulation was cut with the same length (2-3 times the circumference of the chamber) and as wide as the chamber is tall. This was used to insulate the sides of the chamber. Finally, four circles of Reflectix of the same diameter as the lid of the chamber were cut to insulate the top and bottom of the chamber. Two of the circles were set on the lid, and a slit was cut in both layers over the hole in the lid. The vacuum inlet of the air ejector feeds through this slit. A thin strip and the circles of insulation are shown in Figure 3.1 .

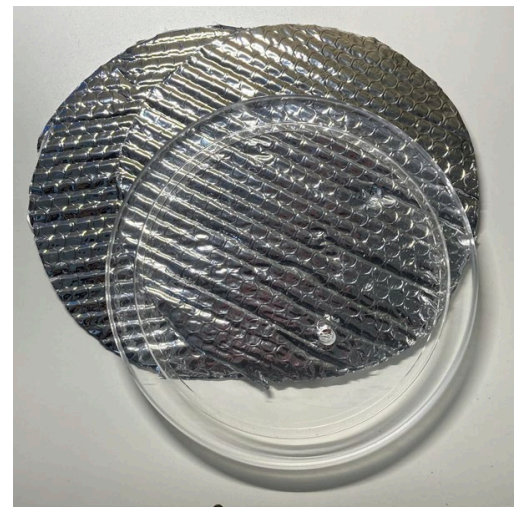

(a)

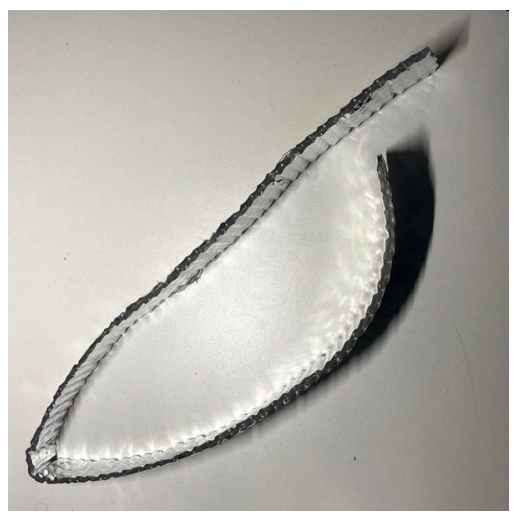

(b) 
Figure 3.1. Insulation was cut to fit the specific vacuum chamber that was purchased. (a) Circles of insulation to cover the base and lid; (b) A strip of insulation to be wrapped around the circumference of the chamber to build an air gap.

Next, the air ejector was assembled. The selected air ejector has 1/8-inch NPT female threads for the air intake and vacuum inlet. The air intake must connect to an air compressor hose, which has a $1 / 4$-inch I/M coupler (often called a quick-connect). The vacuum inlet must connect to the chamber lid and a vacuum gauge. On the vacuum chamber in use, the lid was sized for an M10 vented bolt. The selected vacuum gauge required a $5 \mathrm{~mm}$ hose barb. Rather than purchase and plumb together several connectors to accomplish this, the connectors were 3-D printed. The internal geometry of the printed connectors is shown in Figure 3.2. To create the composite parts, the hose barb and I/M coupler were modeled in FreeCAD v0.18 [59] based on measurements of purchased parts. FreeCAD offers straightforward profile-modeling tools, but OpenSCAD [60] has a broader, more robust set of thread libraries, so OpenSCAD v2019.05 was used to compose the final parts. Kirshner's 'threads' library [61], available under the GNU GPL 3.0 [62] was used to create ISO-standard metric threads for the M10 nut. Corona688's 'tmsthread4 library [63], available under the Creative Commons Attribution NonCommercial (CC-BY-NC) license [64], was used to create NPT threads for both components.

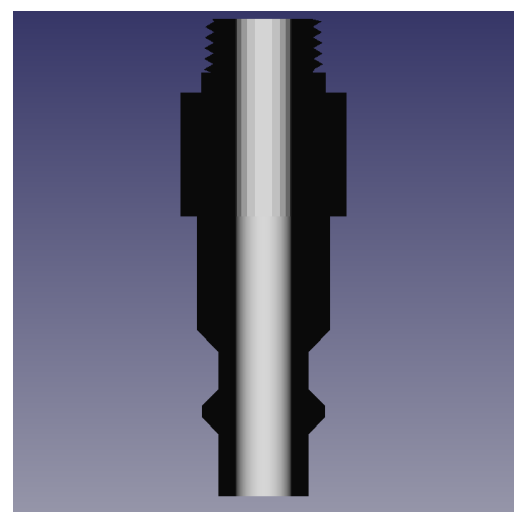

(a)

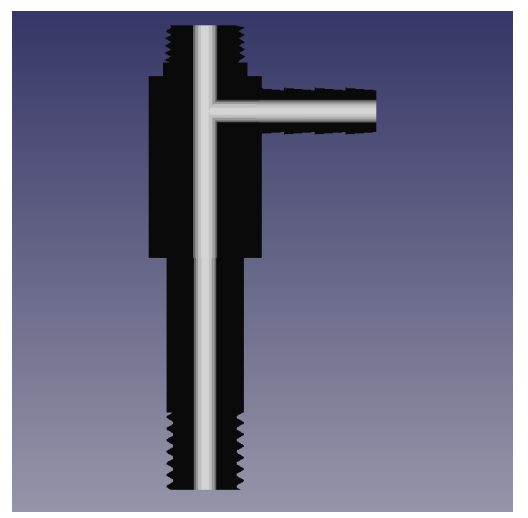

(b)

Figure 3.2. Air ejector connector cross-sections, highlighting internal geometry where air flows. (a) Intake connector, where high pressure air enters the ejector; (b) Vacuum connector, where evacuated air flows from the chamber to the ejector and vacuum pressure is measured.

The design and STL files for each component are available, along with all other data, designs, and documentation, on the OSF repository [45] under the GNU General Public License (GPL) 3.0 [62]. Note that the 3-D printed parts are actually available under CCBY-NC [64] because they use tmsthread4 for the NPT threads. All other files and data are available under GPL 3.0. The original CAD files (OpenSCAD [60] and FreeCAD [59]) are available to allow modification in the case that the parts they attach to are different than those used in this build. The components were 3 -D printed using $1.75 \mathrm{~mm}$ polylactic acid (PLA) filament on an Athena II delta-style RepRap class 3-D printer [65] with a 
$0.4 \mathrm{~mm}$ nozzle. The STL for each component was sliced using Ultimaker Cura v.4.7.1 [66] and the print settings shown in Table 3.2. After printing, supports and brim materials were cleaned off of the prints.

Table 3.2. Print Settings for 3-D Printed Parts (PLA).

\begin{tabular}{cc}
\hline Property & Value \\
\hline Layer Height & $0.2 \mathrm{~mm}$ \\
Wall Thickness & $2 \mathrm{~mm}$ \\
Top/Bottom Thickness & $0.8 \mathrm{~mm}$ \\
Infill & Cubic, $20 \%$ \\
Nozzle Temperature & $210{ }^{\circ} \mathrm{C}$ \\
Print Speed & Infill/Support: $70 \mathrm{~mm} / \mathrm{s}$, Wall: $35 \mathrm{~mm} / \mathrm{s}$ \\
Outer Wall Speed & $35 \mathrm{~mm} / \mathrm{s}$ \\
Retraction & Yes \\
Print Cooling & No \\
Support & Build-plate only, $50 \mathrm{deg}, 15 \%$ density \\
Adhesion Type & Brim \\
\hline
\end{tabular}

Testing has shown that the printed parts can withstand the high pressure $(80 \mathrm{psig})$ at the intake and low pressure (26 inHg vacuum) at the vacuum inlet, and the printed threads work and properly seal at the connections. The connectors were threaded into their respective sockets on the air ejector (Figure 3.3a). To check that the ejector was functional, the vacuum gauge was attached (Figure 3.3b) and 80 psi air was applied to the air intake. Plugging the vacuum connector showed around 24-26 inHg vacuum pressure on the gauge. To resolve issues with a faulty seal on the connectors, adding PTFE thread seal tape can help - note that PTFE tape is only intended for tapered threads [67], so it will not help with the M10 threads.

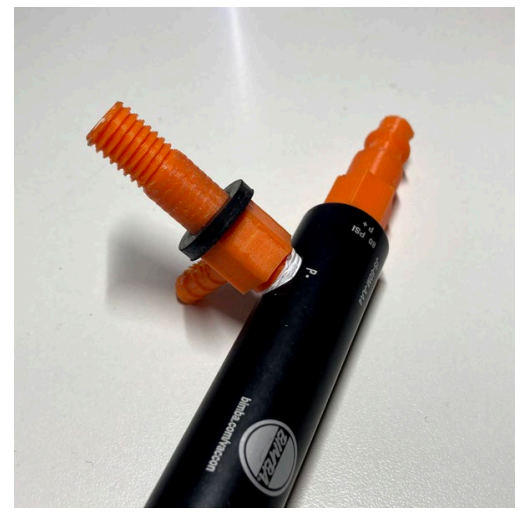

(a)

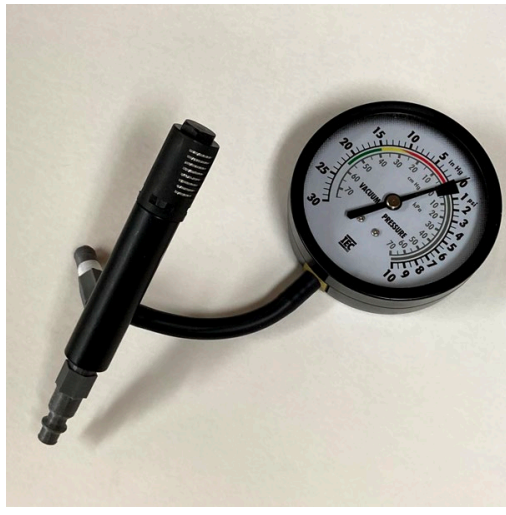

(b)

Figure 3.3. Assemble the air ejector. (a) Thread the 3-D printed connectors into the air ejector, using PTFE tape if necessary; (b) Attach the vacuum gauge to the hose barb. 
With the air ejector assembled and checked, the vacuum inlet was fed through the two circles of insulation. Next, one M10 washer was placed on the inlet (Figure 3.4a), then the inlet was fed through the lid of the vacuum chamber. The other M10 washer was applied and the M10 nut was tightened onto the threads, securing the air ejector and insulation to the lid (Figure 3.4b).

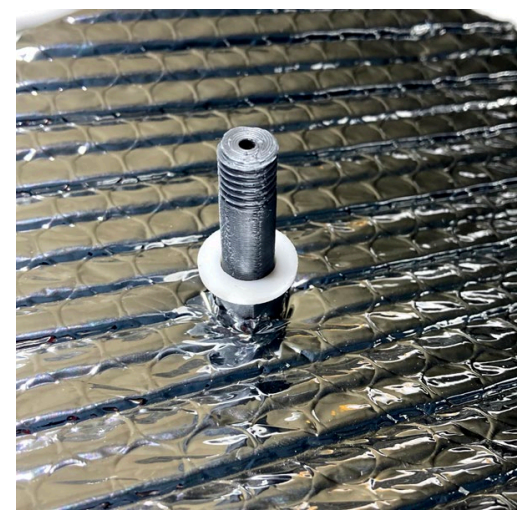

(a)

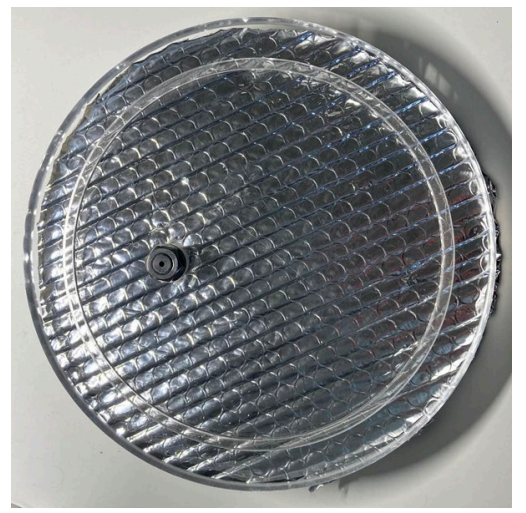

(b)

Figure 3.4. Process to connect the air ejector to the lid. Note that the photographed part is grey here, where it was orange in prior photos. (a) Feeding the ejector through the insulation, then an M10 washer (nylon or rubber); (b) Feeding the ejector through the lid, then another washer, then secure with an M10 nut.

Finally, the insulation was installed on the walls of the chamber. This was done after installing the heating element, allowing the sensor wire and power cable to be fed up through the wall insulation (as shown in Figure 3.5) for strain relief.

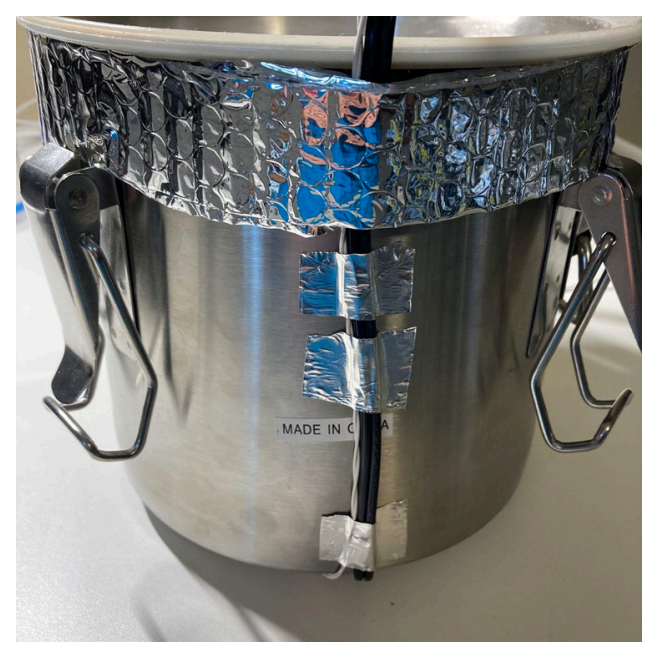

Figure 3.5. If installed, the heater power cable and sensor wires may be fed up the wall of the chamber for strain relief, but this is not required.

To install the wall insulation, the two strips of insulation were wrapped around the top and bottom as shown in Figure 3.6a, securing them to the chamber wall and themselves with flue tape (other adhesives may be used in the event that lower temperatures will be 
used for drying). Next, the large strip of insulation was wrapped around the walls of the chamber, once again using tape to secure it to the already installed layers of insulation, and to itself. The final result is shown in Figure 3.6b.

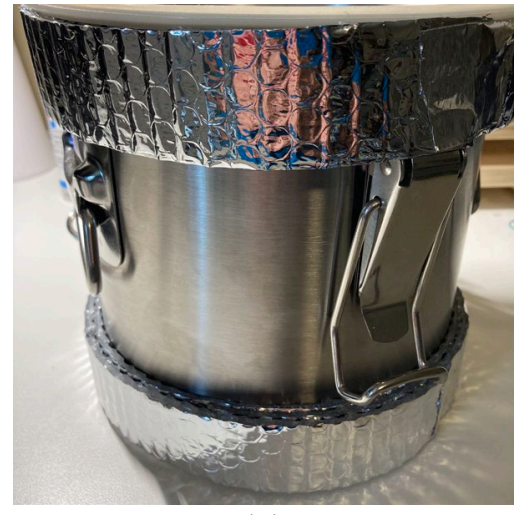

(a)

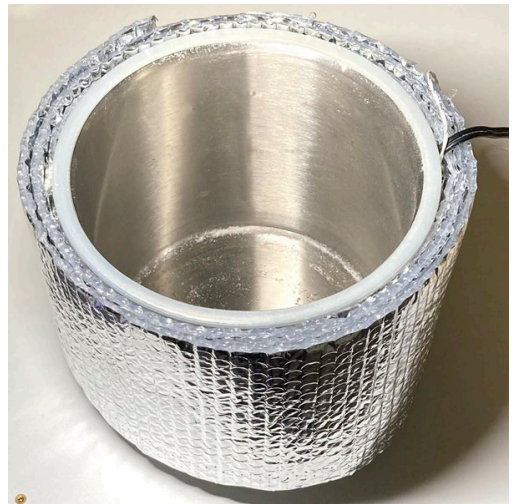

(b)

Figure 3.6. Install insulation on the chamber wall. (a) First, attach strips of insulation to build an air gap and span the protruding components on the chamber walls; (b) Next, install the large piece of insulation, fully covering the walls of the chamber.

The two layers of insulation for the base of the chamber were installed after the installation of the heating element.

\subsubsection{Thermal Control System}

Before building the thermal controller, thermistor calibration measurements were collected to calculate the Beta coefficient of the thermistor. The thermistor's resistance was measured at 0 and $100{ }^{\circ} \mathrm{C}$ by submerging the thermistor in an ice bath and boiling water, respectively. The thermistor was wrapped in flue tape prior to submersion to protect it from short-circuiting in the water. The resistance measurements were recorded and used to calibrate the thermistor as described in 3.2.7.1.

The controller measures the thermistor with a voltage divider, which uses a reference resistance connected to the reference voltage. This resistance is placed in series with the thermistor, which is connected to ground. The voltage at the node connecting the two resistances is measured with an analog pin on the Arduino. This voltage is used to determine the resistance of the thermistor. The value of the reference resistance has a direct impact on the precision of the temperature measurement. Increasing the difference between the measured voltage at the upper and lower temperature bounds increases the number of discrete voltages (therefore, temperatures) that the ADC can measure. The measured voltage, $V_{m}$, for a given voltage divider with thermistor resistance $\mathrm{R}_{\text {th }}$ and reference resistance $\mathrm{R}_{0}$ is calculated using (3.1). This equation assumes the reference voltage is $1 \mathrm{~V}$, meaning that the difference between two measured voltages corresponds to a percentage of an ADC's measurement range.

$$
\mathrm{V}_{\mathrm{m}}=\mathrm{R}_{\mathrm{th}} /\left(\mathrm{R}_{0}+\mathrm{R}_{\mathrm{th}}\right)
$$


The difference between $V_{m}$ at the upper $\left(R_{t h 1}\right)$ and lower $\left(R_{t h 0}\right)$ operating resistances should be maximized. Using the simplified Steinhart-Hart equation (3.2), the thermistor resistance was estimated at the upper and lower operating temperature. The nominal resistance and temperature, $100 \mathrm{kOhm}$ at $25{ }^{\circ} \mathrm{C}$, were used as $\mathrm{R}_{1}$ and $\mathrm{T}_{1}$, respectively. The nominal Beta value, $\beta$, provided by the manufacturer, 3950, was also used [50]. A calibrated value could be used instead, after completing the thermistor calibration as described in 3.2.7.1.

$$
\beta=\ln \left(\mathrm{R}_{1} / \mathrm{R}_{2}\right) /\left(1 / \mathrm{T}_{1}-1 / \mathrm{T}_{2}\right)
$$

With these values estimated, selecting $\mathrm{R}_{0}$ became a simple optimization problem, with the cost function $\left(\mathrm{C}_{\mathrm{f}}\right)$ in (3.3). The equation is written in such a manner to emphasize that the difference between the two measured voltages should be maximized, making no assumptions about which voltage will be larger. The sign is changed because optimization conventionally seeks to minimize cost.

$$
\mathrm{C}_{\mathrm{f}}=-\left|\mathrm{R}_{\mathrm{th}} /\left(\mathrm{R}_{\mathrm{th}}{ }+\mathrm{R}_{0}\right)-\mathrm{R}_{\mathrm{th} 1} /\left(\mathrm{R}_{\mathrm{th}}+\mathrm{R}_{1}\right)\right|
$$

This cost function was minimized using 'minimize_scalar', which is an optimization method in the SciPy Python library's 'optimize' package [68]. Intuition would suggest that the optimal resistance $\mathrm{R} 0$ has a magnitude on the order of the thermistor resistance, so the initial guess provided to minimize_scalar was the average of the operating bounds. A Python script named 'voltage_divider_optimization.py', which returns the optimal resistance, is available on the OSF repository [45]. It accepts the thermistor beta value and reference temperature-resistance pair, plus the operating temperature range, completing all other computations internally.

The optimization was run for a few operating ranges, shown in Table 3.3. The operating range during design was expected to be 50 to $60{ }^{\circ} \mathrm{C}$, so a $30 \mathrm{kOhm}$ resistor was used. A similar approach to this problem, as well as further explanation on the rationale, have been discussed by Hrisko [69].

Table 3.3. Optimized reference resistances for several operating temperature ranges.

\begin{tabular}{ccc}
\hline $\begin{array}{c}\text { Temperature Range } \\
\left({ }^{\circ} \mathbf{C}\right.\end{array}$ & $\begin{array}{c}\text { Reference Resistance, R0 } \\
(\mathbf{k O h m})\end{array}$ & ADC Range Utilized \\
\hline $50-60$ & 30 & $9 \%$ \\
$60-70$ & 21 & $9 \%$ \\
$70-80$ & 15 & $8 \%$ \\
$50-80$ & 21 & $25 \%$ \\
\hline
\end{tabular}

The thermal control circuit, shown in Figure 3.7, is fairly simple - it only requires a voltage divider, used to measure the thermistor resistance, and a pair of signal wires to control the relay. 


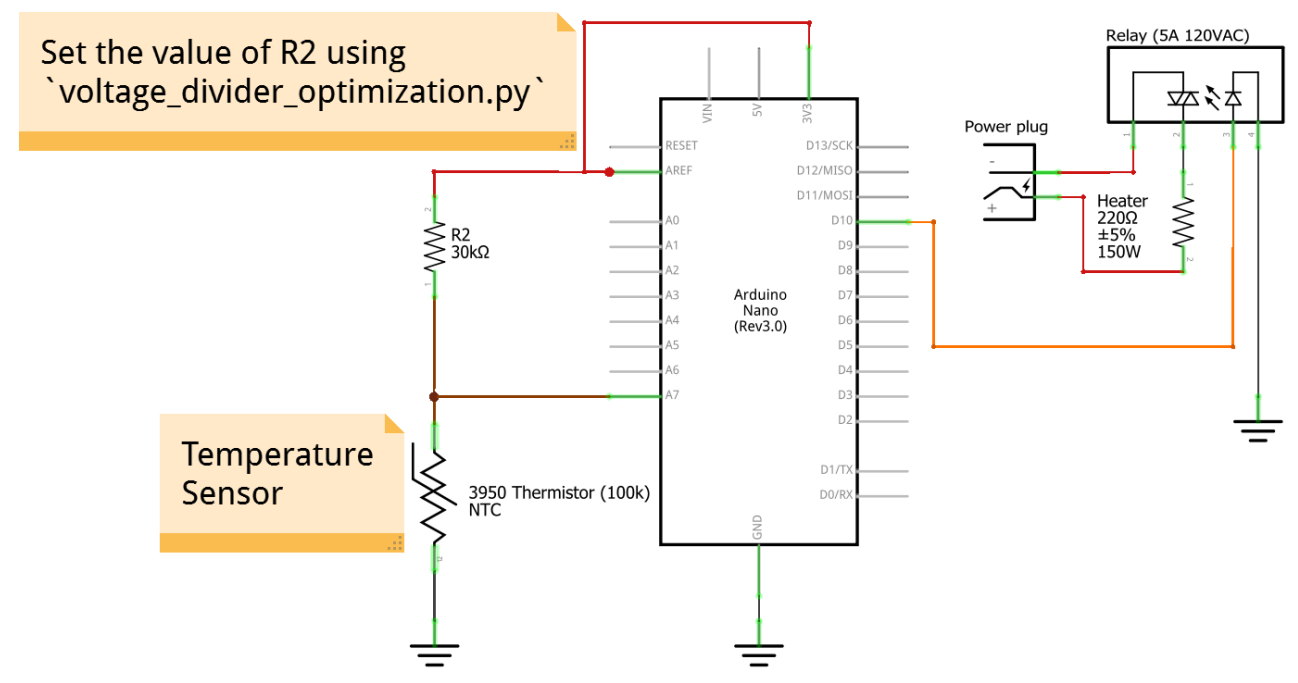

Figure 3.7. The circuit is a voltage divider and a pair of signal wires to control the relay.

The circuit was assembled on a $3 \times 7 \mathrm{~cm}$ solder breadboard. The assembly of that board is detailed in 3.7, and the final circuit is shown in Figure 3.8.

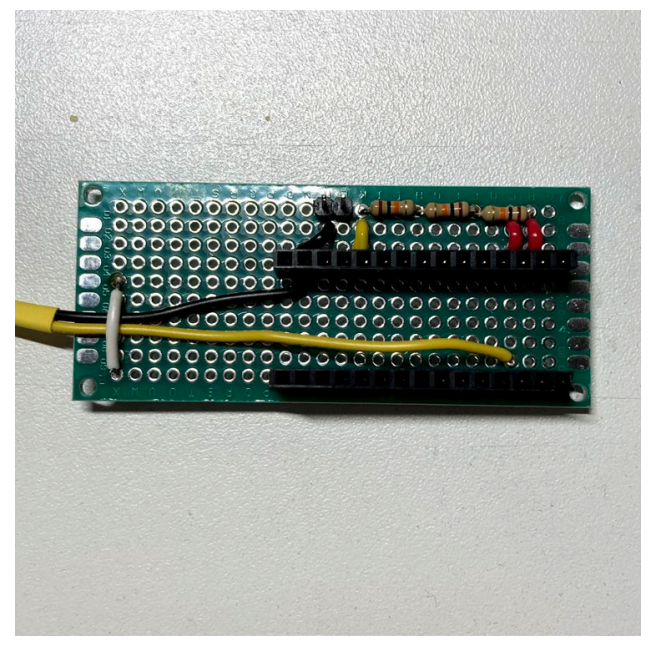

Figure 3.8. The completed circuit is wired to connect to a relay and measure a single thermistor.

With the circuit fully constructed, the dryer firmware (available on the OSF repository [45]) was uploaded to an Arduino Nano, which was then installed in the female header pins with the USB on the column 'A' side of the board (Figure 3.9a). The thermistor was attached to its male header pins and the relay was connected to the signal wires (Figure $3.9 b)$. 


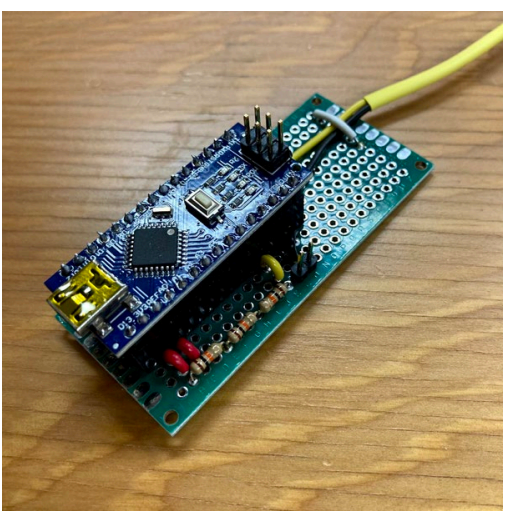

(a)

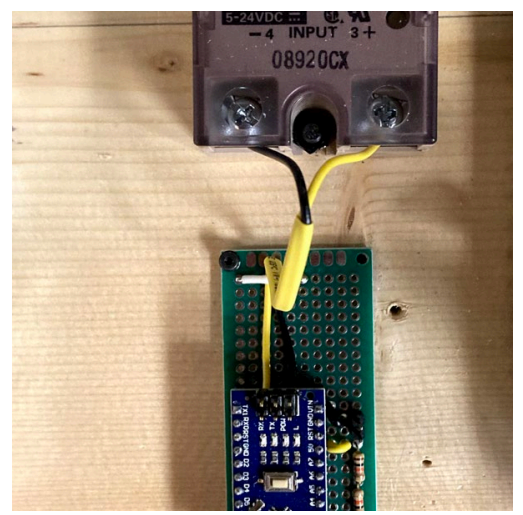

(b)

Figure 3.9. Assembly of the electronics for the thermal controller. (a) The Arduino installed on the circuit; (b) The signal wires attached to the relay.

The heating pad has an adhesive on one side. The transfer tape was removed from the heating pad, revealing the adhesive. Prior to installing the heater on the base of the vacuum chamber, the thermistor bulb was stuck to the middle of the heating pad. Adhering the pad to the base of the chamber secured the thermistor in place (Figure 3.10 ), providing a direct measurement of the heater temperature. Pressure was applied to the heating pad for 60 seconds to allow the adhesive to set.

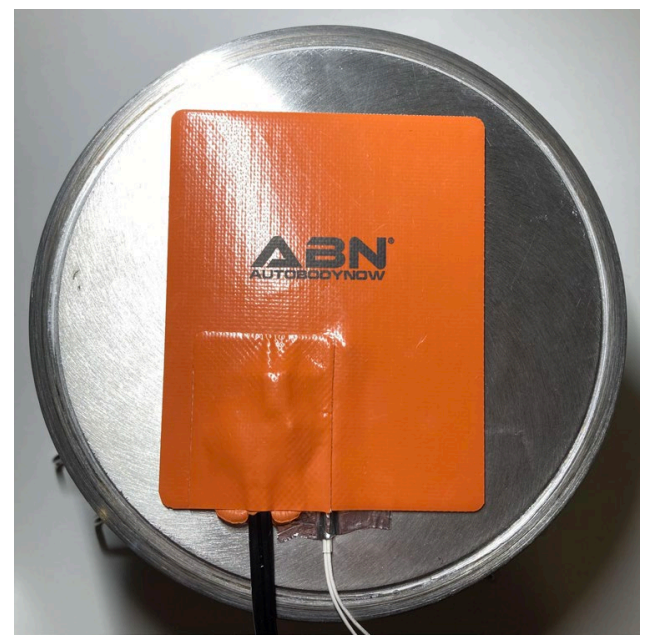

Figure 3.10. The heating element was adhered to the base of the chamber, with the thermistor secured on the interface.

To allow the heater to be controlled by the relay, the heater power cable was cut in half (Figure $3.11 \mathrm{a}$ ). Only one wire needs to be severed - the other may remain intact. In this case, both wires were cut, so one pair of wires was reconnected using a wire nut (Figure 3.11b). 


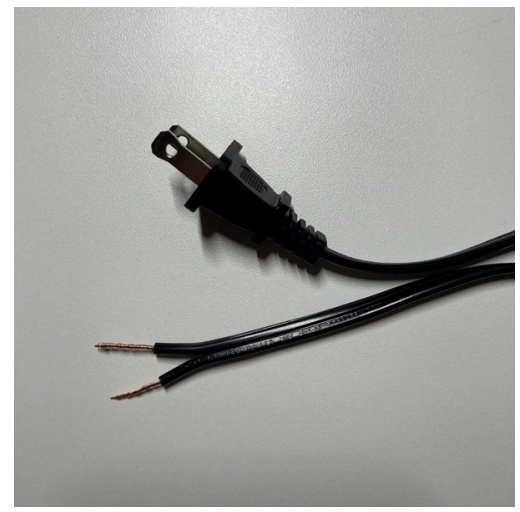

(a)

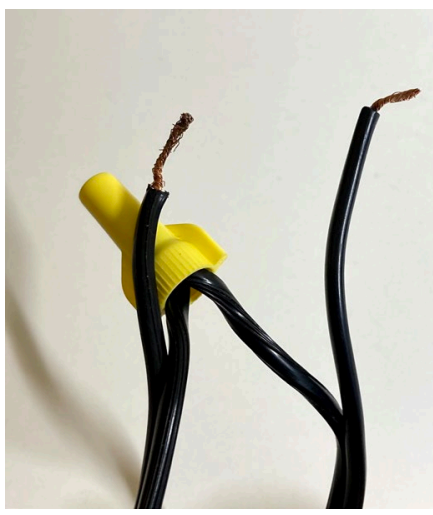

(b)

Figure 3.11. The power cable was prepared for connection to the relay. (a) First, it was cut in half. Only one wire needed to be cut, but both were cut in this case; (b) Next, one pair of wires was immediately re-joined with a wire nut.

The other pair of wire ends were stripped and secured in the load terminals (1 and 2) of the relay, as shown in Figure 3.12.

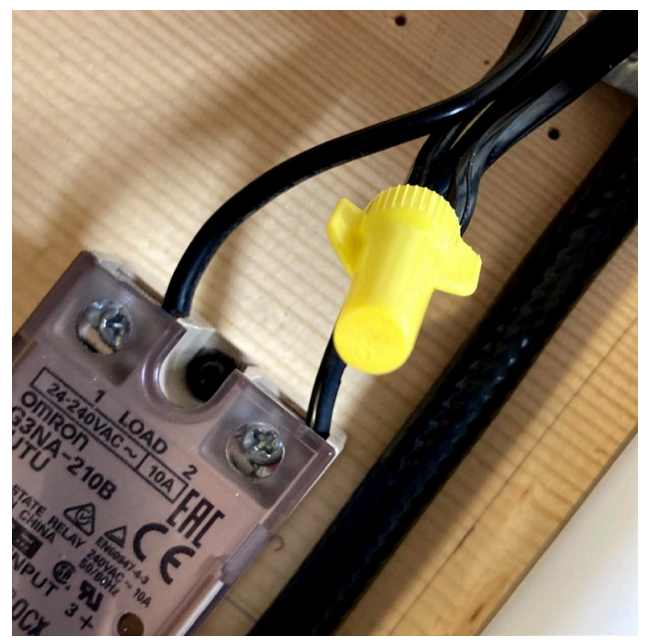

Figure 3.12. The power cable was secured into the load terminals of the relay.

\subsubsection{Finishing the Assembly}

In an effort to tidy wires and make transportation easier, the electronics for the oven were assembled on a wooden frame. The relay was secured with two M4 machine screws, and the circuit was secured with four M2 machine screws. Power was provided to the Arduino and the heater via a power strip, also attached to the board. The final assembly is shown in Figure 3.13. 


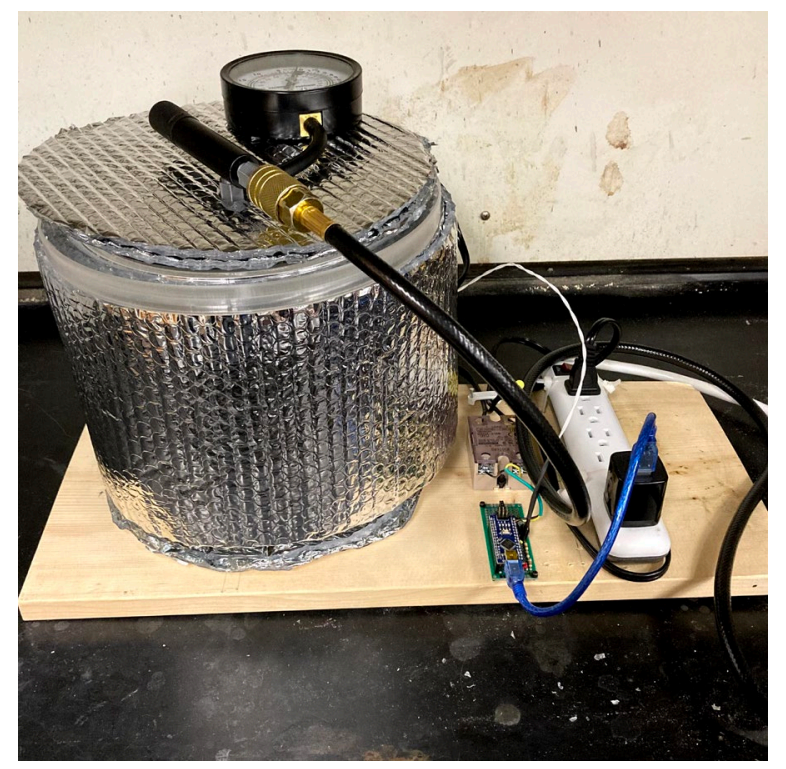

Figure 3.13. The fully assembled vacuum oven.

\subsubsection{Code}

The firmware driving the dryer is fairly straightforward. It uses Salimov's NTC_Thermistor library to handle the simplified Steinhart-Hart equation for computing temperature from the voltage divider measurement [70]. The temperature measurements are read into a 10-sample averaging FIR filter. A longer average could feasibly be used without affecting the dynamics of the system, but this number of averages is memoryefficient for the Arduino and was found to be sufficiently smooth for the purposes of control.

The system tracks the average temperature and uses relay control with no hysteresis to regulate the heater temperature - turning the heater on if the temperature is below the set temperature, and off if it is above. Testing showed that the switching of this relay was sufficiently filtered out inside the chamber, making a more sophisticated controller unnecessary.

Every second, the current time and temperature are printed to serial for the sake of data logging and checking performance. In an effort to prevent catastrophic failure due to a sensor error, the temperature is checked to make sure it is reasonable. In the event that the thermistor open-circuits, the resistance reading should fall to around absolute zero ($273.15 \mathrm{~K})$. Designing on the assumption that the vacuum oven will not be used in a room where the temperature is below $0 \circ \mathrm{C}$, a temperature reading below 0 will force the relay to turn off and throw a message over serial that something looks wrong. This value can be changed to meet different needs, or even to track a predictive model in order to further improve failure-safety. In the opposite event, should the thermistor short-circuit, the controller would read a very high temperature, above the set temperature for the heater, so the relay would naturally turn off. 


\subsubsection{Operation}

Operation of the oven requires a few steps. First, the material to be dried was placed in the oven. Next, the operating temperature was set to meet the limits of the material being dried. The upper limit for a safe operating temperature is two-fold. First, the heating element comes with a thermal fuse set to trip at around $130{ }^{\circ} \mathrm{C}$, meaning that temperature cannot be exceeded. Secondly, the chamber lid is made of acrylic and the manufacturer recommends that it not be exposed to temperatures greater than $60{ }^{\circ} \mathrm{C}$ [27]. With the current design, the temperature gradient on the chamber is significant, making the lid little cause for concern. Improvements to the design which provide more even heating of the chamber must take this into consideration. The current firmware uses a hard-coded set temperature. This value was changed by editing the variable T_set in the firmware, then re-flashing the Arduino. This removed any need for a user interface (such as a display to show, or knobs to vary, the set temperature), though one could be added in the future. Once the temperature was set, the heating element was turned on by providing power to the Arduino and the heater.

With the material loaded and warming up, the only remaining step was to introduce a vacuum. First, the lid was placed on the chamber, and the air compressor hose connected to the compressor and the lid. The manufacturer recommends that this be completed prior to charging the air compressor for safety [31]. Next, the air compressor was turned on and allowed to charge to full capacity. Once the pump had turned off, the pressure regulator was set to provide 80 psig of pressure to the air ejector. Light pressure was applied to the lid until the vacuum gauge started to show a vacuum pressure. At this point, the lid was held in place by air pressure. The ultimate vacuum achieved was around $25 \mathrm{inHg}$, or about $17 \mathrm{kPa}$ absolute. At this pressure, the saturation temperature of water is approximately $57{ }^{\circ} \mathrm{C}[28]$.

The testing discussed in the results offer some guideline for the amount of time required to dry material, but testing should be conducted to determine the appropriate time and temperature for drying specific materials. The test procedure used here offers a framework for determining such drying times.

\subsubsection{Materials for Testing}

The system was tested with both plastics for DRAM and biomaterials as part of a project to convert post-consumer plastic into edible food.

\subsubsection{Plastics}

Recycled polyethylene terephthalate (rPET), which is the most abundant waste plastic, was produced for testing by washing and shredding used water bottles into flakes. PET is a highly hygroscopic material, meaning it tends to absorb water into its chemical structure $[5,71]$. Absorbed moisture can only be removed to the point of equilibrium with the air surrounding the material. This means that simple hot-air drying cannot make the plastic any dryer than the air in the room. This can be a major impediment to successful use of rPET in DRAM as for example simple air drying does not work in humid 
environments. Thus, converting rPET to 3-D printing filament is normally regulated to demonstrations on lab-grade or industrial grade recycling systems using blends $[72,73]$ or composites [74] rather than low-cost open source recyclebots (waste plastic extruders that produce filament) $[75,76]$, which are more appropriate for DRAM. Vacuum drying offers much lower atmospheric moisture content than hot-air drying, meaning it can dry hygroscopic materials more completely than hot-air drying [71]. The rPET offered an opportunity to check for signs of this behavior, limited by the measurement resolution of the balance in use. Prior to testing, the rPET was placed in an unsealed container for a span of several days to provide an opportunity for the plastic to reach an equilibrium of moisture content with the laboratory.

\subsubsection{Biomaterials}

Another approach to recycling PET is to use bio-organisms to convert it to useful products like food. To begin to probe this potential, microorganisms were grown on rPET chemical components. Specifically, terephthalic acid is a building block of polyester plastics such as PET. Various bacteria can use terephthalate as a carbon and energy source $[77,78]$. A bacterial consortium was enriched from compost with the ability to grow to high densities using terephthalate as a sole carbon source. This consortium shows promise for the bioprocessing of terephthalate wastes as well as potential transformations of plastics like PET.

Single cell protein (SCP) is protein that is derived from single celled organisms such as bacteria and algae [79]. SCP has been proposed as an alternative food source to deal with growing food insecurity [80,81]. In order to convert microbial biomass into single cell protein, biomass needs to be treated to both preserve and inactivate the cells. Here the novel drying system is tested as a post-treatment procedure to produce SCP from microbial biomass grown on terephthalate.

Enrichment cultures were set up using a sample of vermicompost, which is a compost that is broken down using worms, from a farm in Calumet, MI, USA (47.211, -88.553). The vermicompost was sampled using a pre-sterilized scoop and was collected in a sterile $50 \mathrm{~mL}$ Falcon tube. The sample was placed on ice and transported back to Michigan Technological University (MTU), where it was stored at $4^{\circ} \mathrm{C}$.

Aerobic cultures were set up in sterile $250 \mathrm{~mL}$ Erlenmeyer flasks using 1 gram of vermicompost soil, $100 \mathrm{~mL}$ of Bushnell-Haas media (0.2 g/L MgSO4, $0.02 \mathrm{~g} / \mathrm{L} \mathrm{CaCl} 2,1$ $\mathrm{g} / \mathrm{L} \mathrm{KH}_{2} \mathrm{PO}_{4}, 1 \mathrm{~g} / \mathrm{L}\left(\mathrm{NH}_{4}\right)_{3} \mathrm{PO}_{4}, 1 \mathrm{~g} / \mathrm{L}$ KNO3, $\left.0.05 \mathrm{~g} / \mathrm{L} \mathrm{FeCl3}\right), 0.25 \mathrm{~g}$ terephthalic acid, $0.25 \mathrm{~g}$ terephthalamide, $2.5 \mathrm{~mL}$ of chemically deconstructed polycarbonate product, and $0.125 \mathrm{~mL}$ of a 1:1:1:1 mixture of $\mathrm{C} 6: \mathrm{C} 10: \mathrm{C} 16: \mathrm{C} 20$ alkenes. The deconstructed polycarbonate product served as a source of bisphenol A. Cultures were placed in a temperature-controlled shaker and incubated at $200 \mathrm{rpm}$ and $30^{\circ} \mathrm{C}$. Cultures were transferred into fresh media of the same composition at a 1:10 dilution once every two weeks. After five transfers, $100 \mu \mathrm{L}$ of material from the enrichment cultures were transferred for growth on disodium terephthalate for biomass production. The consortia was transferred four times in Bushnell Haas medium with $10 \mathrm{~g} / \mathrm{L}$ disodium terephthalate as the carbon source prior to the growth of the consortia for biomass production. 
To produce biomass for the dryer, $500 \mathrm{ml}$ of Bushnell-Haas medium supplemented with $10 \mathrm{~g} / \mathrm{L}$ of disodium terephthalate was inoculated with $5 \mathrm{ml}$ of the culture grown on disodium terephthalate. The culture was grown at $25^{\circ} \mathrm{C}$ for three days. Biomass from the consortium was harvested by centrifugation at $7,500 \mathrm{x}$ g for 10 minutes. The supernatant was removed, and the biomass was stored at $4{ }^{\circ} \mathrm{C}$ until use for testing the open source vacuum oven in drying.

\subsubsection{Testing}

\subsubsection{Thermistor Calibration}

The thermistor was calibrated using resistance measurements gathered at three known temperatures, shown in Table 3.4. These measurements were used to calibrate the full Steinhart-Hart equation (3.4), which has three coefficients and is solved with a system of three equations as described in the literature [41].

Table 3.4. Thermistor calibration measurements.

\begin{tabular}{cc}
\hline Temperature $\left({ }^{\circ} \mathbf{C}\right)$ & Reference Resistance, $\mathbf{R 0}(\mathbf{k O h m})$ \\
\hline 25 & 100. \\
0 & 310 \\
100 & 7.1 \\
\hline
\end{tabular}

$$
\mathrm{T}=1.0 /\left(\mathrm{k}_{\mathrm{a}}+\mathrm{k}_{\mathrm{b}} * \log \left(\mathrm{R}_{\mathrm{t}}\right)+\mathrm{k}_{\mathrm{c}} * \log \left(\mathrm{R}_{\mathrm{t}}\right)^{3}\right)
$$

Three combinations of the measurements in Table 3.4 were then used to compute Beta three times. The thermistor curves for those three Beta values, plus the nominal value, were compared with the full Steinhart-Hart equation. These computations were completed using a Python script named 'thermistor_calibration.py', available on the OSF repository [45]. The Beta value that provided the least error was selected to calculate temperature on the Arduino.

\subsubsection{Temperature Gradient Measurements}

In order to quantify the temperature gradient resulting from the location of the control temperature sensor, three thermistors were installed on a 3-D printed jig in the center of the chamber. The jig held the thermistors 0,25 , and $50 \mathrm{~mm}$ above the base of the chamber. The thermistor jig is shown in the chamber in Figure 3.14a. These temperatures were measured on a separate microcontroller from that driving the heater. The measurements were taken with rPET material inside the chamber, with the chamber at atmospheric pressure (measuring the temperature under vacuum conditions faced the same challenges as for placing the control thermistor inside) and the lid off. The thermal controller was set to $60{ }^{\circ} \mathrm{C}$, measured at the heating element. This test was completed twice: (1) with only rPET inside the chamber; then (2) with an added metal cylinder (referred to as a heat sink) inside to aid in the distribution of heat to the rPET. The heat sink with the thermistors and rPET is shown in Figure 3.14b. 


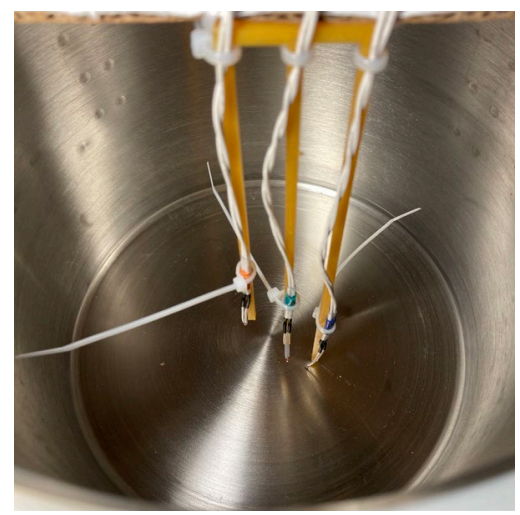

(a)

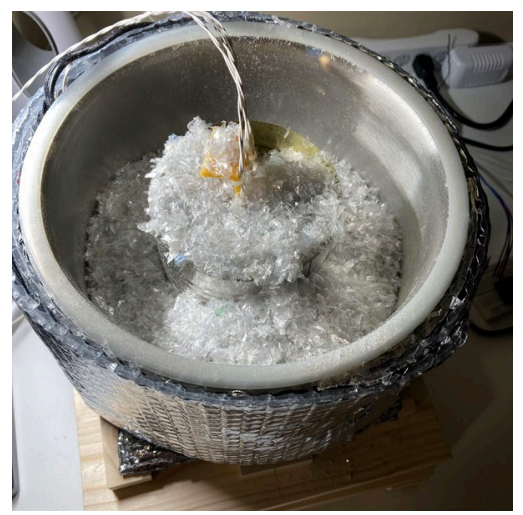

(b)

Figure 3.14. The test setup for measuring temperature gradient. (a) Three thermistors were mounted on a 3-D printed jig, mounting them 0,25 , and $50 \mathrm{~mm}$ above the base of the chamber; (b) For the second test, a heat sink was added in the mix of rPET to aid heat transfer throughout the material.

An additional set of tests was completed in which the thermistor mounted at $0 \mathrm{~mm}$ (on the base, inside the chamber) was used to drive the relay controller. This was tested with no material in the chamber, and with rPET in the chamber. Once again, the lid was off, meaning the thermistor was exposed to convective interference that would otherwise not be present under vacuum. The temperatures of the heater and the $25-$ and $50-\mathrm{mm}$ thermistors were recorded. This configuration offered an approximation of how the controller would behave if the control thermistor were mounted inside the chamber while under vacuum. The results indicated the necessary heater temperature to reach an inside surface temperature of $60{ }^{\circ} \mathrm{C}$, and aid in estimating the inside surface temperature for a given heater set temperature.

\subsubsection{Drying Rate Comparison}

The vacuum oven was tested against an off-the-shelf food dehydrator [82], which would be classified as a typical hot-air dryer. To quantify the dehydration of each sample, the mass of each sample was measured prior to and throughout testing using an open source precision mass balance, calibrated using a $100 \mathrm{~g}$ standard mass with a rated precision of 5 mg [26]. During each test, the energy consumption of the vacuum oven and the food dehydrator were each measured using multimeters, measuring energy in kWh to 0.01 $\mathrm{kWh}$ precision [83]. The vacuum oven's heating and vacuum (air compressor) energy consumption were measured separately to help identify where the majority of energy consumption occurs.

Testing was first conducted on small samples (10 g) of rPET to determine a set temperature for the oven. The samples were measured on a $100 \mathrm{~g}$ load cell with a reported measurement accuracy (standard deviation) of $5 \mathrm{mg}$ [26]. Three $10 \mathrm{~g}$ samples were prepared in petri dishes with known masses. $300 \mathrm{mg}$ of water was added to each sample, bringing the total mass to $10.3 \mathrm{~g}$, such that the added water comprised approximately $3 \%$ of the mass. The first sample was placed in the center of the vacuum oven base, which was set to $70 \circ \mathrm{C}$. The second was placed on the lowest shelf of the food 
dehydrator, also set to $70 \circ \mathrm{C}$ (its maximum temperature). All trays provided with the dehydrator were left in the assembly during testing, in accordance with the operating instructions [82]. The third sample was left on a counter to act as a control. Every 15 minutes, each sample was weighed while left in its petri dish. This ensured that no flakes of plastic or droplets of moisture could be left behind in transfer between containers. The mass of the empty petri dish was subtracted from the measured mass, recording only the mass of the plastic and water. This was repeated with the vacuum oven set to $80 \circ \mathrm{C}$.

Next, large samples $(350 \mathrm{~g})$ of rPET were tested with the vacuum oven set to $80{ }^{\circ} \mathrm{C}$. At this point, small sample testing had showed that both dryers acted faster than a control, so no control was kept for further measurements. Each large sample was measured on a $5 \mathrm{~kg}$ load cell with a reported measurement accuracy (standard deviation) of $20 \mathrm{mg}$ [26]. The samples once again had 3\% water by mass added, constituting 10.8 grams of water. Given the size of the sample, the plastic was stirred after the addition of water to encourage an even distribution of water throughout the sample. These samples were too large to keep in containers within each dryer, so measurements were made less often (every 60 minutes), and the plastic was transferred from each dryer to a separate bowl for each measurement. In the vacuum chamber, two metal cylinders were included as heat sinks during the large sample tests. In the dehydrator, all trays were filled with plastic. The lowest tray had a dense metal screen material installed in order to minimize the loss of small pieces of plastic into the base of the dehydrator.

To test the drying of biomass, samples of approximately $500 \mathrm{mg}$ were measured into petri dishes, once again with the mass of the container measured beforehand. The bio-mass was expected to be about $60 \%$ water by mass, so no water was added. These samples were measured every 15 minutes while being dried.

\subsubsection{Filament Drying}

In order to verify the effects of the vacuum oven on Fused Filament Fabrication (FFF) 3D print quality, two tests were run. First, two small $(\sim 30 \mathrm{~g})$ samples of PLA filament were taken from a single spool of PLA filament which had been stored in a humid environment. The first sample was used to print the vacuum connectors from Table 3.1. The second sample was dried using the printer, set to $60{ }^{\circ} \mathrm{C}$, for two hours, then used to print the same parts. Both prints were completed on the same day and with the same printer. The resulting prints were photographed for visual comparison.

Second, a $1 \mathrm{~kg}$ spool of PLA filament which had been stored in a laboratory was dried in the vacuum oven, set to $80 \circ \mathrm{C}$. The mass of the spool was measured every half hour until no changes in mass were observed. This mass was measured using the same $5 \mathrm{~kg}$ load cell as was used for the large samples of rPET.

\subsubsection{Economic Analysis}

In order to quantify the cost of this device, its total cost is divided between the two components. Here only the material costs are considered. Several components are available for bulk purchase, providing enough material for multiple builds of this device, 
or use on other devices. This means that the device has two costs. First, the up-front cost, that which would be paid to build a single vacuum oven, counting all leftover material as cost for the build. This is the summation of the bulk price, $\mathrm{Pb}$, of each component.

Second, the effective cost, Ce, which accounts for only the material used in the build. This is computed by (3.5), which corrects the bulk price using the bulk volume, $\mathrm{V}_{\mathrm{b}}$, and the volume consumed, $\mathrm{V}_{\mathrm{c}}$. The computation of the bulk and effective cost is completed in the BOM spreadsheet, available on the OSF repository [45].

$$
\mathrm{C}_{\mathrm{e}}=\mathrm{P}_{\mathrm{b}} * \mathrm{~V}_{\mathrm{c}} / \mathrm{V}_{\mathrm{b}}
$$

Where the correction is applied to each component's cost. The cost of 3-D printed materials (listed in Table 3.1) is the mass of the part times the cost of plastic per kg. The cost of the PLA filament for the Athena II was U.S. \$19/kg [84].

\subsection{Results}

\subsubsection{Thermistor Calibration}

The calculated full-model coefficients for the Steinhart-Hart equation are shown in Table 3.5 .

Table 3.5. Steinhart-Hart full model calibration.

\begin{tabular}{cc}
\hline Coefficient & Value \\
\hline $\mathrm{Ka}$ & $6.9 \mathrm{e}-4$ \\
$\mathrm{~Kb}$ & $2.1 \mathrm{e}-4$ \\
$\mathrm{Kc}$ & $1.3 \mathrm{e}-7$ \\
\hline
\end{tabular}

These were used to compute the thermistor curve shown in Figure 3.15.

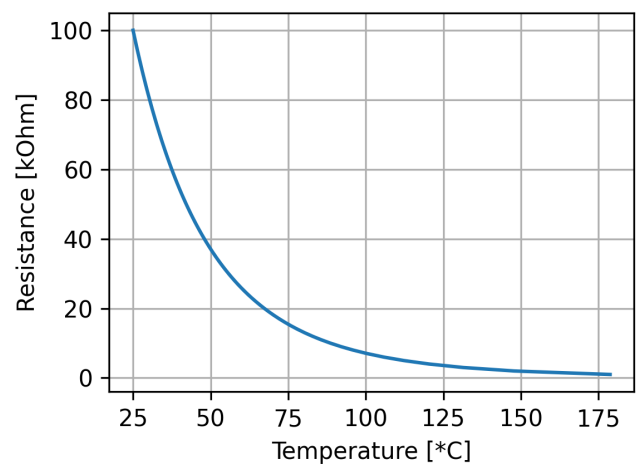

Figure 3.15. The full Steinhart-Hart equation yields this temperature curve, showing the thermistor's sensitivity decreasing as temperature increases.

The computed Beta values are shown in Table 3.6. These were used to generate comparison curves with the full model. The difference between the full and simplified 
model for each Beta value is shown in Figure 3.16. The calibration shows that a Beta equal to 3920 or 3950 is the best option, maintaining error within $1{ }^{\circ} \mathrm{C}$ for the expected operating range. Since 3950 is the nominal value, it was used in recognition of potential error in measuring the resistance and the actual temperature of the thermistor during the calibration measurements.

Table 3.6. Steinhart-Hart simplified model calibration.

\begin{tabular}{cc}
\hline Temperatures Used & Beta \\
\hline 25,0 & 3690 \\
0,100 & 3850 \\
25,100 & 3920 \\
Nominal & 3950 \\
\hline
\end{tabular}
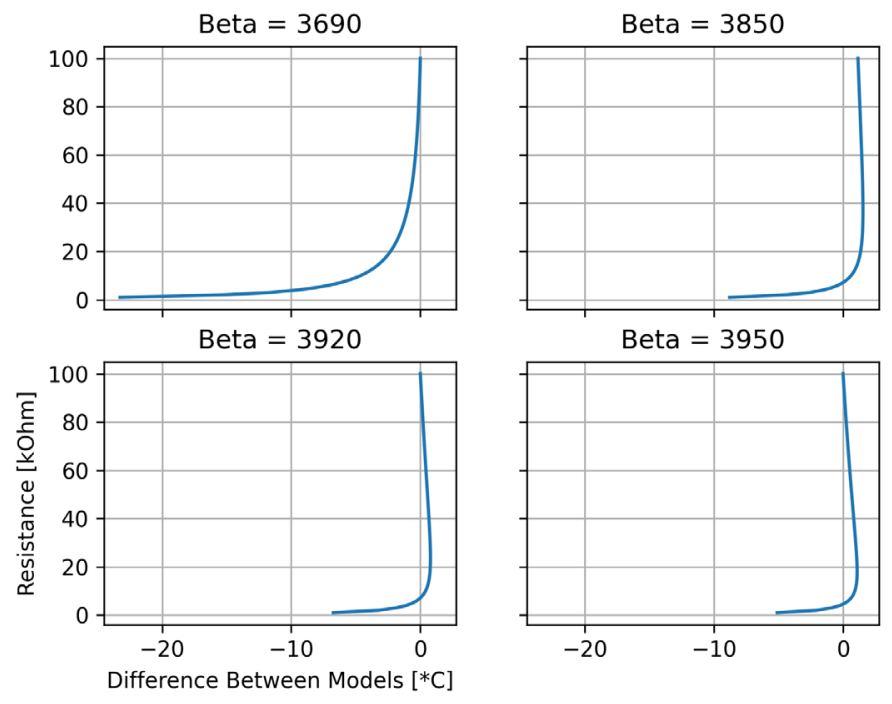

Figure 3.16. The calculated Beta values have varying accuracy as a function of resistance. These curves show that Beta of 3920 or 3950 maintain error within $1{ }^{\circ} \mathrm{C}$ for the majority of the temperature range under assessment.

\subsubsection{Temperature Gradient Testing}

The measured temperature gradient in the chamber with and without an added heat sink is shown in Figure 3.17. The three thermistors show a very steep gradient and slow rise for the temperature in the chamber. The measurements indicate that a heat sink amidst loosely packed material greatly increases the rate of warming throughout the chamber. 


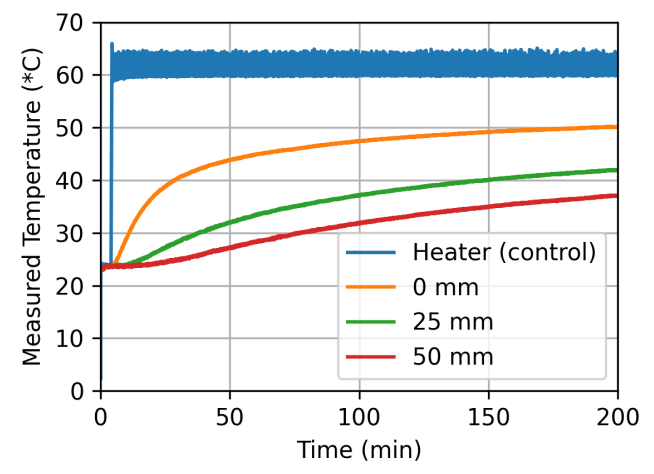

(a)

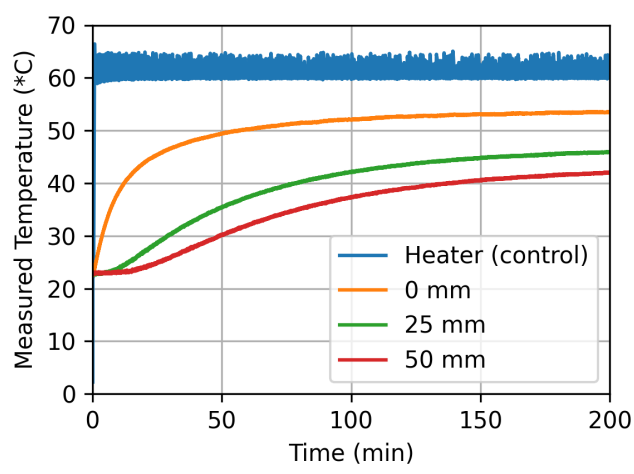

(b)

Figure 3.17. Temperature gradient measurements. (a) Chamber loosely packed with rPET; (b) Chamber loosely packed with rPET, plus a metal cylinder, referred to as a heat sink.

The measured temperature gradients while using the inside $(0 \mathrm{~mm})$ thermistor to drive the controller are shown in Figure 3.18. With no material in the chamber, the heater had to maintain an average temperature of $79{ }^{\circ} \mathrm{C}$ to keep the inside surface of the chamber at 60 -C. Note that the chamber was open to atmospheric air, allowing convective heat loss. After the addition of rPET (still at atmospheric pressure), the heater temperature averaged around $68 \circ \mathrm{C}$ in order to maintain an inside surface temperature of $60 \circ \mathrm{C}$. Note here that the PET had not reached a steady state temperature after one hour of testing. The gradient is likely exacerbated under vacuum conditions because convective heat transfer is no longer possible, restricting heat transfer to only conduction. These results suggest that a set temperature of 70 to $80{ }^{\circ} \mathrm{C}$ on the heater should yield an oven temperature (only at the inside surface of the base) of $60 \circ \mathrm{C}$ (slightly above the saturation temperature of water at the measured vacuum pressure). The addition of bulk material to the oven slows the transfer of heat upward through the chamber, apparently by reducing the rate of convective loss. This observation highlights two possibilities. First, introducing a vacuum will virtually eliminate convective loss inside the chamber, meaning the temperature gradient between the heater and the inside surface of the chamber should be small; and, second, the bulk material acts as insulation, meaning heat transfer in the absence of convection will likely be slow, resulting in a significant temperature gradient on large samples under vacuum. This temperature gradient is likely to negatively affect the rate of drying as the top layer of large samples will not reach the saturation temperature of water. 


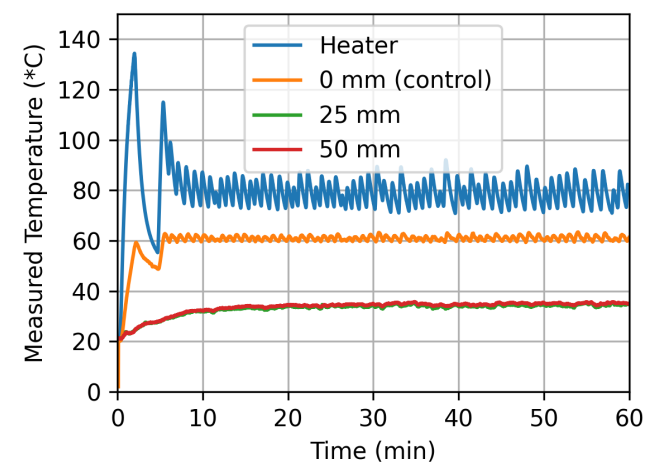

(a)

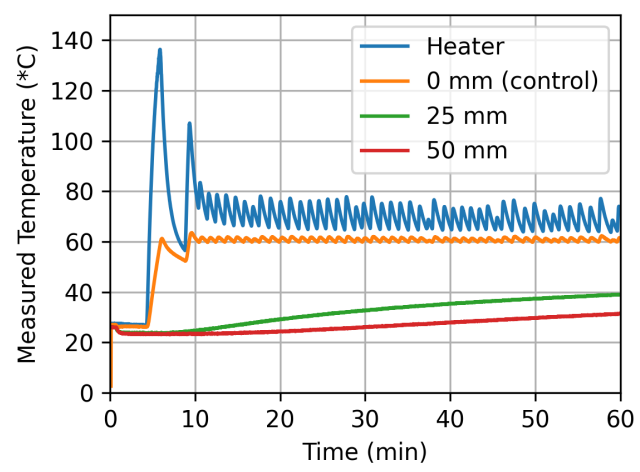

(b)

Figure 3.18. Temperature gradient measurements with the inside surface temperature used for control. (a) Chamber empty and open to the air, average heater temperature 79 -C at steady state; (b) Chamber loosely packed with rPET, average heater temperature 68 $\circ \mathrm{C}$ at steady state.

\subsubsection{Drying Tests}

The results of the drying tests are shown as a computed value - the percentage of the initial mass $\left(\mathrm{p}_{\mathrm{im}}\right)$ remaining at each measurement, calculated using (3.6). This serves two purposes. First, it corrects for any differences in initial mass between each sample, thus correcting out vertical offsets in the data. Second, it highlights the percentage of mass lost in terms of the percentage moisture content. The rPET tests each had 3\% water by mass added to the sample prior to testing, and every sample (except for the large vacuum oven sample, which was cut short) showed a reduction in mass to below the initial 'dry' mass, indicating that additional moisture was removed from the plastic during drying. Since each curve is scaled to a percentage mass, error bars are included to indicate uncertainty $\left(m_{e}\right)$ in the mass measurement. The error is shown as a percent of the measured mass $\left(p_{\mathrm{mm}}\right)$, calculated in (3.7), hence smaller masses have a larger percentage of uncertainty, because they approach the magnitude of the measurement error of the load cell.

$$
\begin{aligned}
& \mathrm{p}_{\mathrm{im}}=\mathrm{m}_{\mathrm{m}} / \mathrm{m}_{\mathrm{i}}[\%] \\
& \mathrm{p}_{\mathrm{mm}}=\mathrm{m}_{\mathrm{e}} / \mathrm{m}_{\mathrm{i}}[\%]
\end{aligned}
$$

Where $\mathrm{m}_{\mathrm{i}}$ is initial mass and $\mathrm{m}_{\mathrm{m}}$ is measured mass.

During all drying tests, the ultimate vacuum achieved in the vacuum oven was approximately $25 \mathrm{inHg}$ vacuum (around $17 \mathrm{kPa}$ absolute), which reduced the saturation temperature of water to around $57 \circ \mathrm{C}$. The results of small sample tests are shown in Figure 3.19. At $70 \circ \mathrm{C}$, the sample in the vacuum oven was considered dry after 75 minutes, and the dehydrator sample was considered dry after 60 minutes, making the vacuum oven $25 \%$ slower than the dehydrator at this setting. This corresponds with what was expected from the temperature gradient tests - a set temperature of $70 \circ \mathrm{C}$ may not have brought the inside of the chamber up to the saturation temperature of water, limiting the rate of evaporation. Increasing to $80{ }^{\circ} \mathrm{C}$ ensured that the samples were warm enough 
to cause rapid drying. In this case, the vacuum oven sample was dried after 45 minutes, while the dehydrator (still set to $70{ }^{\circ} \mathrm{C}$ ) took 75 minutes, making the oven $40 \%$ faster in this test, and $25 \%$ faster than the dehydrator on either $10 \mathrm{~g}$ sample tested. The increased set temperature caused a significant improvement in the oven's performance, though gradient testing shows it is likely that the material in the oven was still at a lower temperature than the dehydrator. The dehydrator settings were not changed between these two tests, yet they show a significant variation between the two tests. This variation was not explored in testing, but could be due to laboratory humidity, or placement of the sample inside the dehydrator the distribution of added water on the plastic. In both cases, the energy consumption of the dehydrator over the span of the 90-minute test was about $0.36 \mathrm{kWh}$. The vacuum oven consumed 0.61 (Figure $3.19 \mathrm{~b}$ ) and $0.55 \mathrm{kWh}$ (Figure $3.19 \mathrm{~d}$ ), respectively. The difference between these is directly related to the number of times the air compressor cycled to refill its tank. The energy required to fill the compressor tank from empty was included in the total energy on the first test only, accounting for $0.04 \mathrm{kWh}$ of the difference in total energy consumed. These results show that the vacuum oven consumes about $70 \%$ more energy than the dehydrator for a given amount of time.

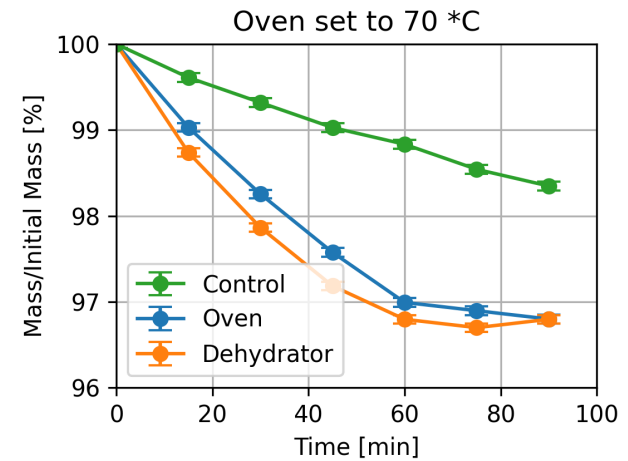

(a)

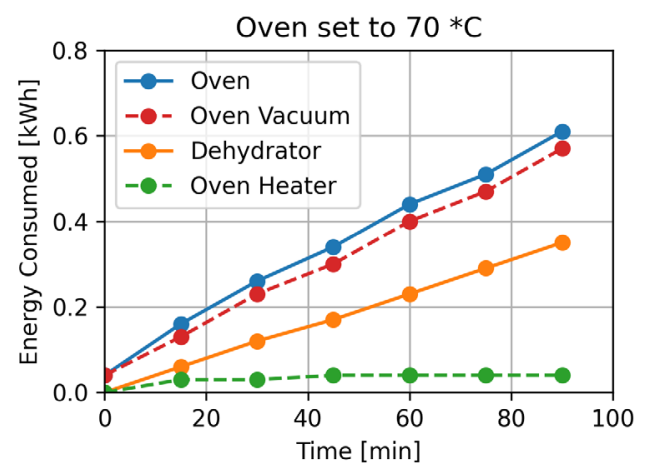

(c)

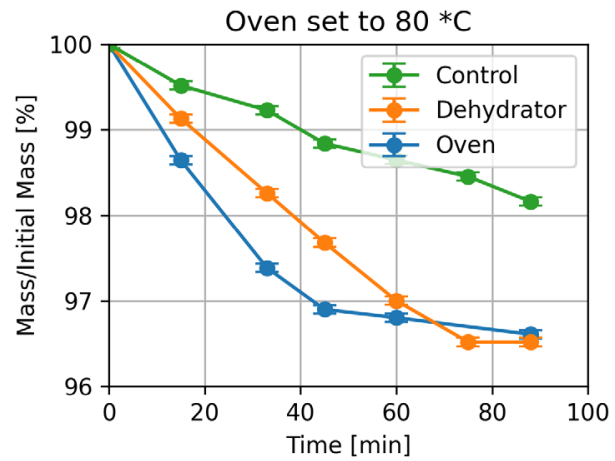

(b)

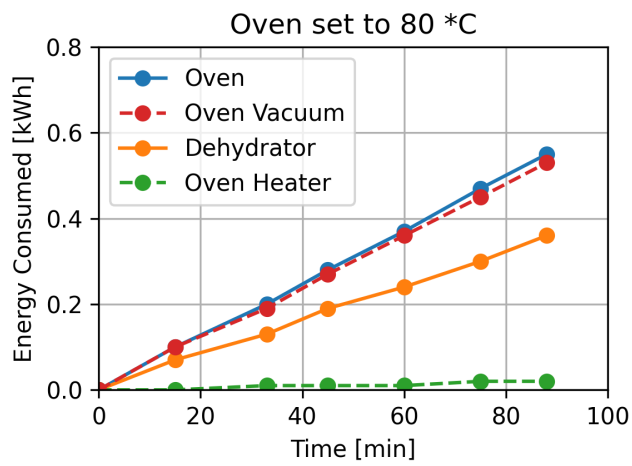

(d)

Figure 3.19. Dehydration measurements on small $(10 \mathrm{~g})$ samples of rPET. Increasing the oven temperature from 70 (left) to $80{ }^{\circ} \mathrm{C}$ (right) caused a $40 \%$ decrease in drying time. (a) Sample percent initial mass with the vacuum oven set to $70{ }^{\circ} \mathrm{C}$. The vacuum oven was $25 \%$ slower than the dehydrator at this setting; (b) Sample percent initial mass with the vacuum oven set to $80{ }^{\circ} \mathrm{C}$ and the dehydrator still set to $70{ }^{\circ} \mathrm{C}$. In this case, the vacuum 
oven was $40 \%$ faster than the dehydrator. (c) The energy consumed during the $70 \circ \mathrm{C}$ test. The dashed lines are summed in the blue aggregate energy for the oven. (d) The energy consumed during the $80 \circ \mathrm{C}$ test.

The results of the large sample rPET test are shown in Figure 3.20. The immediate conclusion drawn from this test was that the vacuum oven cannot handle large volumes of water. During the test, condensation was observed on the lid and upper wall of the vacuum chamber. Condensation indicates that the lower portion of the oven was heating sufficiently to evaporate moisture, but the top of the oven was cool enough to allow water to recondense before being evacuated from the chamber. Given time, the trend shows that the oven could remove all the present moisture. The food dehydrator, however, proved to be significantly faster when drying large samples. The energy measurements for this test are shown in Figure 3.20b. The energy data, consistent with other results, show that the vacuum oven consumes about twice as much energy as the food dehydrator with a vast majority (96\%) of the energy going to the air compressor in order to maintain vacuum pressure in the chamber.

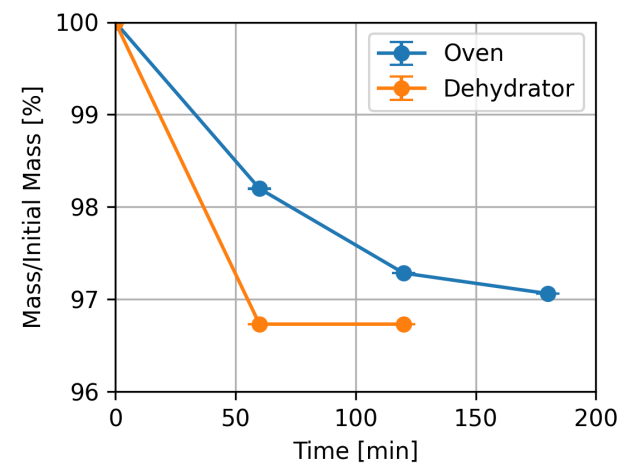

(a)

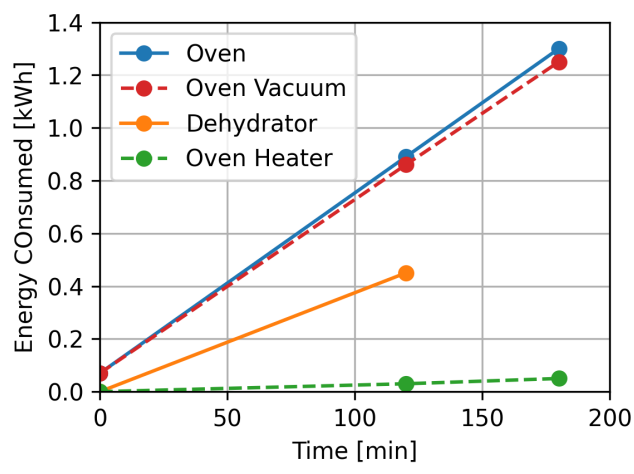

(b)

Figure 3.20. Dehydration of large $(350 \mathrm{~g})$ samples of rPET. This test was cut short because it clearly indicated that the vacuum oven could not manage the large mass of plastic and water. (a) The percentage of initial mass as a function of time. (b) The energy consumption of each device. The energy consumption of the vacuum is shown as an aggregate of the two components, the heater and the vacuum.

Further testing of oven drying efficiency was performed on small samples of microbial biomass. The $500 \mathrm{~mL}$ culture grown on disodium terephthalate produced $2 \mathrm{~g}$ of biomass by wet weight after three days of growth. Approximately $1 \mathrm{~g}$ of biomass was dried, with $500 \mathrm{mg}$ tested in each dryer. The culture is shown before and after dehydration in Figure 3.21 . 


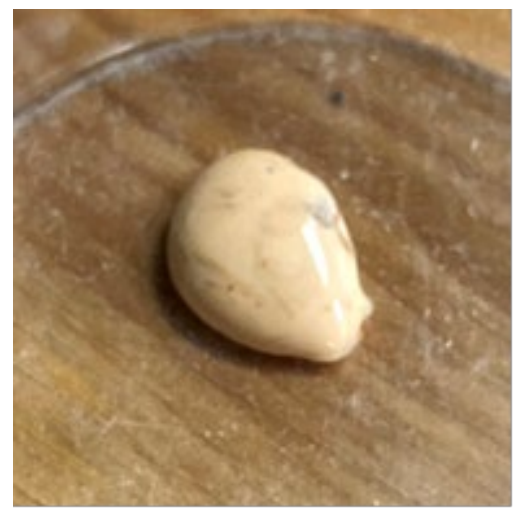

(a)

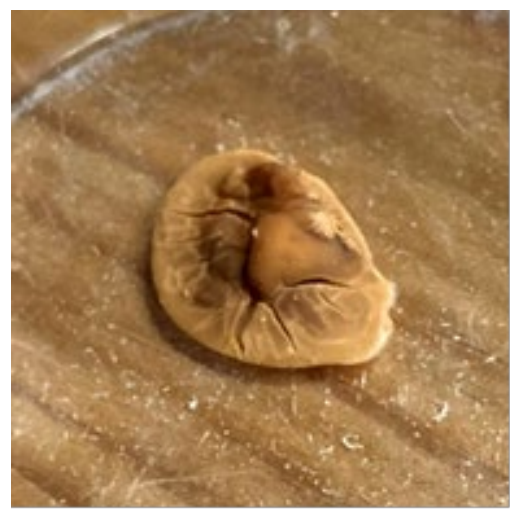

(b)

Figure 3.21. $500 \mathrm{mg}$ samples of the consortium were dehydrated in a petri dish. (a) The wet culture, prior to drying; (b) The fully dehydrated culture.

The results of using the open source vacuum oven for biomass dehydration on these samples are shown in Figure 3.22. These results show the vacuum oven dehydrating the biomass at a much higher rate than the food dehydrator. The oven settled out in about 75 minutes, while the dehydrator took 150 minutes. The small masses (around $500 \mathrm{mg}$ ) under test likely contributed to the performance of the vacuum oven. The sample sizes also result in larger measurement uncertainty from the reported accuracy of the load cell, as indicated by the error bars. The observed difference in the final percent of initial mass is expected to be a result of the biomass sample being non-homogeneous. After completion of the test, the sample from the dehydrator was moved to the vacuum oven in order to check if it could remove more moisture than the dehydrator had. No additional loss of mass was measured after 15 minutes, meaning there is no evidence to suggest that the vacuum oven dried the biomass more completely than the dehydrator.

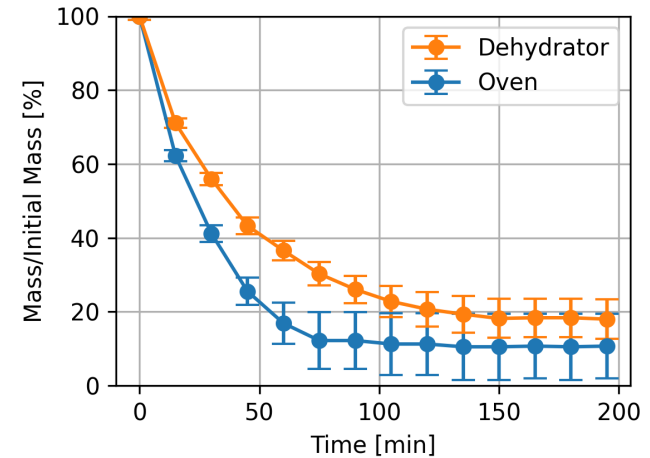

(a)

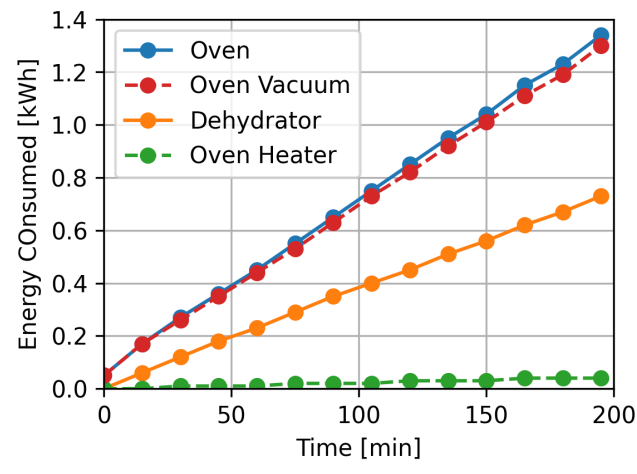

(b)

Figure 3.22. Dehydration measurements on consortium of microorganisms. (a) Percent initial mass for each sample; (b) energy consumption. The dashed oven vacuum and oven heater curves were summed to form the aggregate oven curve.

These test results are summarized in Table 3.7. They show that the vacuum oven is more efficient than the food dehydrator (with regard to time and energy) for small samples 
when the set temperature is $80{ }^{\circ} \mathrm{C}$. The dehydrator proved to be more efficient when handling larger masses of water due to the uneven heating in the chamber.

Table 3.7. Summary of the dehydration test results.

\begin{tabular}{cccc}
\hline Test Description & Device & Drying Time & Energy Consumed \\
\hline \multirow{2}{*}{$10 \mathrm{~g} \mathrm{rPET}, 70{ }^{\circ} \mathrm{C}$} & Vacuum Oven & 75 minutes & $0.51 \mathrm{kWh}$ \\
& Dehydrator & 60 minutes & $0.23 \mathrm{kWh}$ \\
\hline \multirow{2}{*}{$10 \mathrm{~g} \mathrm{rPET}, 80^{\circ} \mathrm{C}$} & Vacuum Oven & 45 minutes & $0.28 \mathrm{kWh}$ \\
& Dehydrator & 75 minutes & $0.30 \mathrm{kWh}$ \\
\hline \multirow{2}{*}{$350 \mathrm{~g} \mathrm{rPET}$} & Vacuum Oven & $180+$ minutes & $1.30+\mathrm{kWh}$ \\
& Dehydrator & 60 minutes & $0.45 \mathrm{kWh}$ \\
\hline \multirow{2}{*}{ Biomass } & Vacuum Oven & 75 minutes & $0.55 \mathrm{kWh}$ \\
& Dehydrator & 150 minutes & $0.56 \mathrm{kWh}$ \\
\hline
\end{tabular}

\subsubsection{Filament Drying}

The print results from the two filament samples are shown in Figure 3.23. The dried filament shows smooth layers, good adhesion, and a smooth surface finish. The un-dried filament resulted in a very porous print with poor layer adhesion. Popping noises were observed during the print; this was likely the effect of water evaporating from the plastic as it was heated in the nozzle.

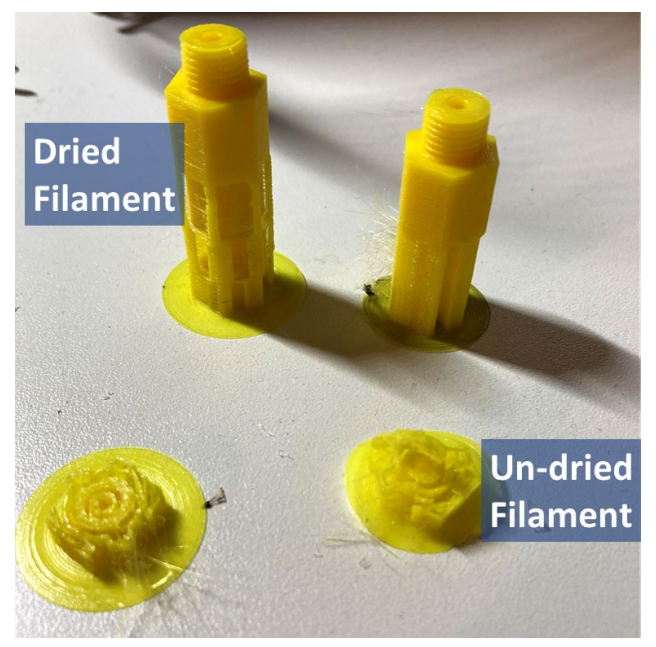

Figure 3.23. The dried filament showed significantly stronger layer adhesion and smoother surface finish.

The measured filament dehydration results show a decrease in mass of about $0.4 \mathrm{~g}$ from the initial mass of $1245.7 \mathrm{~g}$. The change in mass is shown in Figure 24. The small decrease in mass is likely due to the low humidity of the environment in which the filament had been stored. During testing, measurement variation related to the placement 
of the filament on the balance was observed, causing measurement variations on the order of $0.2 \mathrm{~g}$. This is indicated with error bars in Figure 3.24.

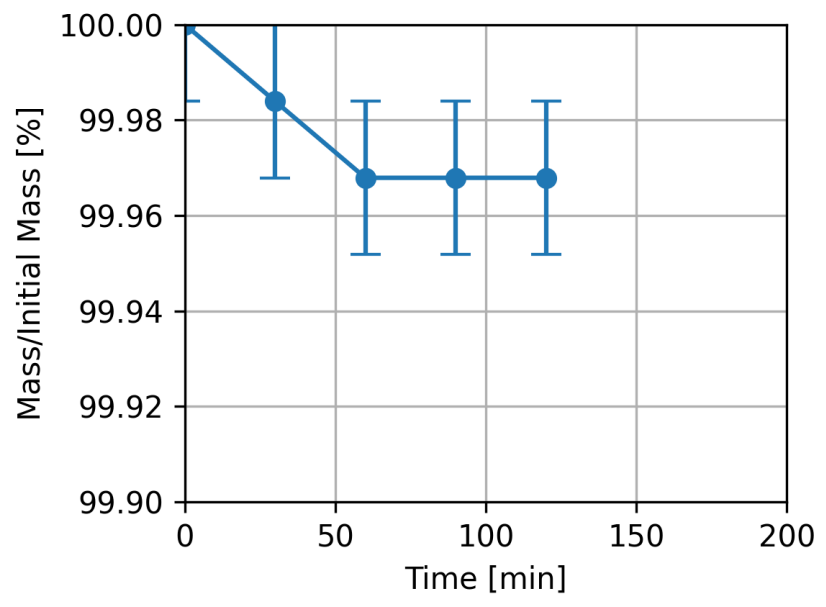

Figure 3.24. A $1 \mathrm{~kg}$ spool of PLA filament was dried in the vacuum oven. The change in mass was around $0.4 \mathrm{~g}$, compared to the initial mass of $1245.7 \mathrm{~g}$.

\subsubsection{Economic Analysis}

The up-front (total cost for all materials) and effective (corrected for bulk purchases) cost of each component and the total material cost of the vacuum oven are shown in Table 3.8. Note that these prices exclude taxes and the cost of shipping, which is now often free from many Internet vendors. Nor does it include labor costs for the FOSH, which have been discussed previously [85]. The majority of the material cost comes from the vacuum system - the chamber, air ejector, and air compressor comprise \$268 of the cost together. Pre-ownership of an air compressor would reduce the total costs by about $\$ 100$. Similarly, the air ejector could potentially be 3-D printed, which would yield somewhere on the order of $\$ 75$ in additional savings.

Table 3.8. Cost breakdown of the vacuum oven.

\begin{tabular}{ccc}
\hline Component & $\begin{array}{c}\text { Up-Front Cost as } \\
\text { purchased (USD) }\end{array}$ & Effective Cost (USD) \\
\hline Heater & $\$ 116.60$ & $\$ 58.17$ \\
Vacuum & $\$ 316.12$ & $\$ 298.39$ \\
Total & $\$ 432.72$ & $\$ 356.56$ \\
\hline
\end{tabular}

\subsection{Discussion}

The test results presented here indicate that the open source vacuum oven successfully works as a drying device. The performance of the oven depends largely on the size of the sample being dried, the moisture content of the sample and the temperature set point of the heater. Laboratory size samples of $10 \mathrm{~g}$ and less were dried within 75 minutes, while 
larger samples on the order of magnitude to support DRAM took upwards of 3 hours to completely dry. Uneven heating in the chamber had negative effects on drying rate while removing large amounts of water from a material because it caused evaporated water to condense on other surfaces in the chamber, rather than leave the chamber via the air ejector. It is suspected that this issue is more closely related with the volume of water being removed, rather than the mass of material being dried, so fairly dry materials that simply need a deeper dry (e.g., rPET materials for DRAM) can be dried in this oven. Heating could be made more even by installing flexible or band heaters around the body of the vacuum chamber, which would decrease the vertical temperature gradient. Additional testing could be done to verify this in a range of commercial DRAM materials and conventional filaments. Further study on a wider range of heater temperatures can also be considered.

It was expected that the dryer would remove a greater volume of water from a hygroscopic material like rPET when compared to a simple hot air dryer. This was not observed in this study. Part of the challenge with this is that the differences in moisture content are fractions of percent initial mass, meaning a large sample or a more sensitive mass measurement device must be used to make the difference measurable. In addition, many tests would be required to overcome the uncertainty of variation in the initial moisture content of samples. In order to gather compelling evidence that this behavior is achievable with this oven, a longer study with more carefully controlled samples must be conducted to gather statistically significant data on the exact moisture content in the material after drying, which should be measured using a moisture analyzer. This is left for future work, because the vacuum oven was shown to be more than adequate for the target applications and case studies presented here.

The open source vacuum oven presented in this study was compared for total effective cost to similar drying solutions available on the market in Table 3.9. Table 3.9 shows the cost of each dryer, and the percent savings $\left(\mathrm{P}_{\text {save }}\right)$ achieved by the open source vacuum oven given by:

$$
\mathrm{P}_{\text {save }}=\left(\left(C_{\text {commercial }}-C_{\text {osvo }}\right) / C_{\text {commercial }}\right)[\%]
$$

Where $\mathrm{C}_{\text {commercial }}$ is the cost of a commercial dryer and COSVO is the material cost of the open source vacuum oven. To clarify, a $\mathrm{P}_{\text {save }}$ of $40 \%$ indicates that the open source oven costs $40 \%$ less than the commercial option. Its effective cost is less than $20 \%$ the cost of available vacuum dryers on the market.

Table 3.9. Cost comparison of the open source vacuum oven with other devices on the market.

\begin{tabular}{ccc}
\hline Type of Dryer & Cost & Percent Savings \\
\hline Vacuum Oven [86] & $\$ 2920.00$ & $87.8 \%$ \\
Vacuum Filament Drier [24] & $\$ 2295.00$ & $84.5 \%$ \\
Freeze Dryer [23] & $\$ 2195.00$ & $83.8 \%$ \\
\hline
\end{tabular}


Although, the open source vacuum oven is significantly less costly than commercial vacuum ovens, it costs more than commercially available hot-air solutions that range from $\$ 45$ [82] to $\$ 70$ [20]. Thus, the open source vacuum oven provides an economical solution when vacuum drying is needed. The savings shown in Table 3.9 are consistent with expected savings of about $87 \%$ observed in a recent review of scientific FOSH [85].

There are several ways the current system could be augmented to improve performance. First, more even heating could be obtained with a higher power heating system covering a greater surface area of the chamber. Energy testing showed that this could be accomplished with a minimal effect on the total energy consumption of the device. Aided distribution of heat throughout sparse or weakly-conductive material has been shown to be possible with the addition of metal cylinders in the chamber to transfer heat from the source throughout the chamber. The accuracy of the temperature could be better monitored if the temperature measurement were performed inside the chamber. This could be accomplished by hermetically sealing a cable or connector in the wall of the chamber to keep a sensor inside the chamber under vacuum, or perhaps a temperature sensor could be housed in the lid to avoid metal work. Multiple temperature measurements could be used to track temperature gradients in the chamber and avoid hotspots which could damage portions of the material being dried. Alternatively, material could be pre-heated in a more thermally conductive environment, prior to being introduced to vacuum. This approach is used in the plastics industry and would likely improve the oven's effectiveness on large samples [25].

In order to reduce energy consumption, the vacuum system could be improved. Once ultimate vacuum is reached, the efficiency of the air ejector becomes poor - the consumption of compressed air is the same, but very little air is removed from inside the chamber. With the addition of a valve on the vacuum connector, the air ejector could be cycled on and off, turning back on once the evaporation of water causes the absolute pressure to increase inside the chamber. Air ejectors could also be connected in series (multi-stage) in order to achieve a deeper ultimate vacuum, which could reduce the minimum set temperature for effective drying and increase the drying rate when operating at higher temperatures.

One of the strengths of modern open hardware is the ability to replicate the hardware from digital designs that themselves can be manipulated with libre software [87,88]. This approach enables more scientists to have access to state-of-the-art equipment [89] and thus encourages democratization of production [90]. In this design costs of fittings were greatly reduced using 3-D printed parts. A natural continuation of that design that would leverage the open hardware paradigm to a greater degree is to have an OpenSCAD fully 3-D printable version of the air ejector. Due to the tolerances necessary, material extrusion printing may not be adequate because of layer thickness limitations, but there are open source SLA-based 3-D printers that are low cost and accessible. Such advancements would also reduce the cost barrier to exploring multi-stage air ejectors to achieve a deeper ultimate vacuum and presumably better performance. 


\subsection{Conclusions}

This study has successfully designed, built and tested an open source vacuum oven for low-temperature drying. The system was shown to be effective at drying both recycled plastic and biomaterials, drying at a higher rate than a hot-air dryer for small samples or for samples with low volumes of water. The vacuum oven can be constructed for around $20 \%$ of the cost of a commercial vacuum dryer. It has several laboratory-scale applications including dehydration of microorganisms, drying plastic for DRAM, and chemical processing.

\subsection{Appendix A - Vacuum Selection}

Small off-the-shelf vacuum chambers are typically expected to be evacuated with a rotary vane vacuum pump. A two-stage oil-sealed rotary vane vacuum pump can achieve an ultimate absolute pressure on the order of $0.01 \mathrm{~Pa}$ [91]. Off-the-shelf vacuum chambers often come plumbed with a vacuum gauge and ball valves sized to connect to such a pump - some even ship with the pump itself [92]. Rotary vane vacuum pumps have some drawbacks, however, as they are meant to evacuate air with low moisture content ('dry air'). Any water vapor passing through the pump can condense in the pump's oil, limiting how deep of a vacuum the pump can pull (referred to as 'ultimate vacuum') and potentially causing premature failure of the pump [93]. When evacuating a system known to have water in the system, two methods are broadly used to keep water out of the oil.

The first is by running the evacuated air through a condenser before it reaches the pump [94]. This adds extra hardware to the vacuum system and requires very low temperatures to rapidly condense water out of the air. The second method is to us a 'gas ballast' - a valve in the vacuum pump that adds atmospheric air to the evacuated air, aerosolizing any condensed water so it gets carried out of the pump, rather than collecting in the oil. The unfortunate side-effect of this is that oil also gets aerosolized, requiring an oil trap to recapture oil and prevent respiratory hazards as well as rapid loss of oil [93].

An oil-sealed rotary vane vacuum pump is not the only option for drawing a vacuum. Other styles of mechanical vacuum pumps, more suited to dealing with moisture in the evacuated air, exist, though they cost significantly more than a rotary vane pump [94]. On the same price scale as a rotary vane pump, and well suited to evacuating air with moisture or other gases, is the air ejector, which was selected for this study.

\subsection{Appendix B - Wiring Instructions}

The pads on the breadboard are labeled on a grid system, rows 1-10 and columns A-X. This grid system is used to indicate locations of connections. In the case of hookup wires, each end of the wire connects to two pads - the pad where the wire is secured, then the pin that the wire is actually connected to. Each end of the wire is labeled in this manner: 'pad to insert into : pad to solder to'. An example is shown in Figure 3.25. 


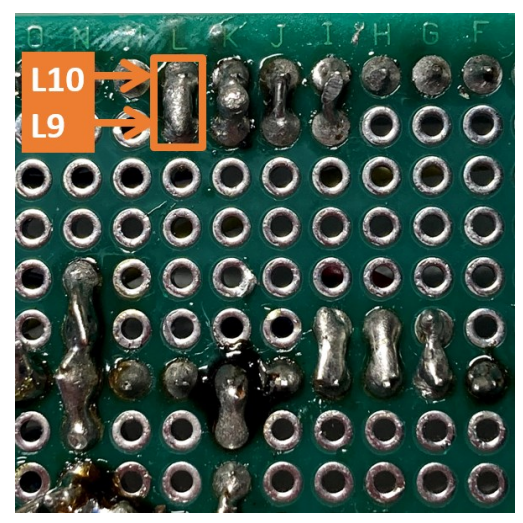

Figure 3.25. Illustration of wire solder labels. The top row is row 10, and it contained a row of header pins. A wire needed to connect to the header pin at L10. To do this, the wire was soldered into L9, then bent over onto L10 (which was already occupied by a header pin) and soldered in place. This wire would be labeled 'L9 : L10'.

First, the $15 \times 1$ female header pins were installed on row 10, columns A-O and row 4, columns A-O. These mount the Arduino Nano, with the USB port on the 'A' side. Next, the $2 \times 1$ header pins were installed in row 1, L-M. These headers receive the thermistor's pre-installed connector.

Next, the reference resistor was soldered into pads B1 and K1:L1. Since three $10 \mathrm{kOhm}$ resistors were in use, they were installed to span that gap, shorted together along the way. This created the voltage divider, with the measurement node at K1:L1.

Two $20 \mathrm{~mm}$ red wires were used to connect power to the reference resistor at the top of the voltage divider. The first wire was connected to B3:B4 and B2:B1. The second was connected to $\mathrm{C} 3: \mathrm{C} 4$ and $\mathrm{C} 2: \mathrm{B} 1$. This provided 3.3V, which is cleaner (less noisy) than $5 \mathrm{~V}$ on an Arduino, to the voltage divider and the ADC reference pin on the Arduino [69]. One $20 \mathrm{~mm}$ yellow wire was connected to $\mathrm{K} 3: \mathrm{K} 4$ and $\mathrm{K} 2: \mathrm{K} 1$, connecting the measurement node to A7 on the Arduino. To ground the divider, a $20 \mathrm{~mm}$ black wire was connected to N3:N4 and M2:M1.

Finally, a $160 \mathrm{~mm}$ black wire was connected to N5:N4 with the other end open. Similarly, a $160 \mathrm{~mm}$ yellow wire was connected to C9:C10. These wires connect to the input terminals ( 3 and 4 ) on the solid-state relay (Figure 3.9b). The completed circuit is shown in Figure 3.8 .

\subsection{References}

1. Parikh, D.M. Solids Drying: Basics and Applications. Chemical Engineering 2014.

2. Jansen, J. Plastics Engineering - January 2015 - Plastic Failure Through Molecular Degradation. Plastics Engineering January 2015.

3. Bozzelli, J. Injection Molding: You Must Dry Hygroscopic Resins. Plastics Technology 2010. 
4. Calovini, L. Everything You Need to Know About Desiccant Drying. Shini USA 2016.

5. Stoughton, P. How to Dry PET for Container Applications. Plastics Technology 2014.

6. Månsson, A. How Moist Filaments Will Screw up Your 3D-Printing. 3 DPrinterChat 2016.

7. Dertinger SC, Gallup N, Tanikella NG, Grasso M, Vahid S, Foot PJ, Pearce JM. Technical pathways for distributed recycling of polymer composites for distributed manufacturing: Windshield wiper blades. Resources, Conservation and Recycling 2020, 157. https://doi.org/10.1016/j.resconrec.2020.104810

8. Sanchez FA, Boudaoud H, Camargo M, Pearce JM. Plastic recycling in additive manufacturing: A systematic literature review and opportunities for the circular economy. Journal of Cleaner Production 2020, 264. https://doi.org/10.1016/j.jclepro.2020.121602

9. Little, H.A.; Tanikella, N.G.; J. Reich, M.; Fiedler, M.J.; Snabes, S.L.; Pearce, J.M. Towards Distributed Recycling with Additive Manufacturing of PET Flake Feedstocks. Materials 2020, 13, 4273. https://doi.org/10.3390/ma13194273

10. Li, P.; Ramaswamy, S.; Bjegovic, P. Pre-Emptive Control of Moisture Content in Paper Manufacturing Using Surrogate Measurements. Transactions of The Institute of Measurement and Control - TRANS INST MEASURE CONTROL 2003, 25, 3656, doi:10.1191/0142331203tm070oa.

11. Amos, W.A. Report on Biomass Drying Technology; 1999; p. NREL/TP-57025885, 9548; DOI: 10.2172/9548

12. Resin Expert. How to stabilize Wood - Stabilise wood the right way. Available online: https://resin-expert.com/en/guide/how-to-stabilize-wood (accessed on 27 March 2021).

13. Boundless; 6.14E: Desiccation. In Microbiology; LibreTexts: California, USA, 2021.

14. Vega-Mercado, H.; Marcela Góngora-Nieto, M.; Barbosa-Cánovas, G.V. Advances in Dehydration of Foods. Journal of Food Engineering 2001, 49, 271-289, doi:10.1016/S0260-8774(00)00224-7.

15. The Basics of Resin Drying. Available online: https://www.ptonline.com/knowledgecenter/plastics-drying/drying-basics (accessed on 8 June 2020).

16. Sherman, L.M. Resin Dryers: Which Type Is Right for You? Plastics Technology 2005.

17. Barley, J. Basic Principles of Freeze Drying. Available online: https://www.spscientific.com/freeze-drying-lyophilization-basics/ (accessed on 27 March 2021).

18. Yao, C.; Qian, X.-D.; Zhou, G.-F.; Zhang, S.-W.; Li, L.-Q.; Guo, Q.-S. A Comprehensive Analysis and Comparison between Vacuum and Electric Oven Drying Methods on Chinese Saffron (Crocus Sativus L.). Food Sci Biotechnol 2018, 28, 355-364, doi:10.1007/s10068-018-0487-x.

19. 3DXTech. Drying Instructions. Available online: https://www.3dxtech.com/drying-instructions/ (accessed on 8 June 2020).

20. eSun. EBOX. Available online: http://www.esunchina.net/products/246.html (accessed on 8 June 2020). 
21.3D Print Board. Filament Dryer. Available online: https://3dprintboard.com/showthread.php?27550-Filament-Dryer (accessed on 9 June 2020).

22. CNC Kitchen. Investigating Different Methods of Filament Drying (Dehydrator, Vacuum, Oven \& Desiccant). Available online: https://www.cnckitchen.com/blog/cyo43tzz88uqge65xgwz0wv8yvv3rs (accessed on 30 March 2020).

23. HarvestRight. Home Freeze Dryer. Available online: https://harvestright.com/product/home-freeze-dryer/ (accessed on 30 March 2021).

24. AMTechniques. Vacuum Filament Dryer. Available online: https://www.kickstarter.com/projects/gertjan-mulder/vacuum-filament-dryer (accessed on 30 March 2021).

25. Novatec. Vacuum Dryers. Available online: https://www.ptonline.com/knowledgecenter/plastics-drying/dryer-types/vacuumdryers (accessed on 8 June 2020).

26. Hubbard, B.R.; Pearce, J.M. Open-Source Digitally Replicable Lab-Grade Scales. Instruments 2020, 4, 18. https://doi.org/10.3390/instruments4030018

27. SUNCOO 2 Gallon Stainless Steel Vacuum Chamber for Degassing Urethanes, Resins, Silicones and Epoxies Available online: https://www.amazon.com/SUNCOO-Stainless-Degassing-UrethanesSilicones/dp/B078K9Q3F9 (accessed on 29 March 2021).

28. Yunus A. Cengel; Michael A. Boles Table A-4 Saturated Water. In Thermodynamics: An Engineering Approach 8e; McGraw Hill Education, 2016; pp. 904-905 ISBN 978-93-392-2165-2.

29. Engineering Toolbox. Water - Boiling Points at Vacuum Pressure. Available online: $\quad$ https://www.engineeringtoolbox.com/water-evacuation-pressuretemperature-d 1686.html (accessed on 29 March 2021).

30. INNOVA 3620 Vacuum/Carburetor Fuel Pressure Tester. Available online: https://www.amazon.com/INNOVA-3620-Vacuum-CarburetorPressure/dp/B000EW0KPY (accessed on 30 March 2021).

31. CRAFTSMAN 6-Gallon Single Stage Portable Electric Pancake Air Compressor. Available online: https://www.lowes.com/pd/CRAFTSMAN-6-Gallon-SingleStage-Portable-Electric-Pancake-Air-Compressor/1000595167 (accessed on 30 March 2021).

32. Blatchley, C.G. Selection of Air Ejectors. Schutte \& Koerting.

33. Dandachi, J.M.A. Steam Air Ejector Performance and Its Dimensional Parameters. Doctoral Thesis, Loughborough University, Loughborough, England, 1990. https://hdl.handle.net/2134/7041

34. Hill, G.F.; Sachse, G.W. Venturi Air-Jet Vacuum Ejectors for High-Volume Atmospheric Sampling on Aircraft Platforms. NASA 1992, 45.

35. Hall, N. Nozzle Design - Converging/Diverging (CD) Nozzle. Available online: https://www.grc.nasa.gov/WWW/K-12/airplane/nozzled.html (accessed on 18 August 2020).

36. Hydraulics Pneumatics. Compressed Air Guide: Pull, Don't Push. Hydraulics \& Pneumatics 2014.

37. Fixed-Flow Air-Powered Vacuum Pump. Available online: https://www.mcmaster.com/9997K15/ (accessed on 29 March 2021). 
38. BAPI. Thermistor vs RTD Temperature Measurement Accuracy - Application Note. Available online: https://www.bapihvac.com/application note/thermistorvs-rtd-temperature-measurement-accuracy-application-note/ (accessed on 9 June 2020).

39. Williams, A. Thermistors And 3D Printing. Hackaday 2017.

40. Banzi, M. and Shiloh, M., 2014. Getting started with Arduino: the open source electronics prototyping platform. Maker Media, Inc..

41. Microstar Laboratories. Calibrate Thermistors. Available online: http://www.mstarlabs.com/sensors/thermistor-calibration.html (accessed on 29 March 2021).

42. Amtherm. The Secret to Successful Thermistor Beta Calculations. Available online: https://www.ametherm.com/blog/thermistors/thermistor-beta-calculations (accessed on 7 July 2020).

43. Electronics Tutorials. Thermistors and NTC Thermistors. Available online: https://www.electronics-tutorials.ws/io/thermistors.html (accessed on 22 March 2021).

44. Tempco. Duraband Maximum Watt Densities. Available online: https://www.tempco.com/Tempco/Resources/01-BandResources/DurabandsWattDnsty.pdf (accessed on 30 March 2020).

45. Hubbard, B.; Pearce, J. M. Open source vacuum oven for low-temperature drying Available online: osf.io/vf2b8.

46. Reflectix 16 in. x 25 Ft. Double Reflective Insulation Roll with Staple Tab EdgeST16025. Available online: https://www.homedepot.com/p/Reflectix-16-in-X-25ft-Double-Reflective-Insulation-Roll-with-Staple-Tab-Edge-ST16025/100012574 (accessed on 30 March 2021).

47. 3M High Temperature Flue Tape, High Heat Sealing Tape up to 600 Degrees, 15Foot Roll. Available online: https://www.amazon.com/3M-High-TemperatureFlue-15-Foot/dp/B00004Z4DS (accessed on 30 March 2021).

48. ABN Silicone Heating Pad 120V - 4 x 5 Inch Universal Engine Heater Car Oil Pan Heater Pad, 150W Electric Heater Pad. Available online: https://www.amazon.com/ABN-Automotive-Electric-SiliconeHeating/dp/B077J5DSFJ (accessed on 30 March 2021).

49. G3NA-210B-UTU DC5-24 Omron Automation and Safety. Available online: https://www.mouser.com/ProductDetail/653-G3NA210BUTUDC524 (accessed on 30 March 2021).

50. Creality 3D Printer NTC Thermistor Temp Sensor 100K for Ender 3 / Ender 3 Pro/Ender 5 / CR-10 / CR-10S. Available online: https://www.amazon.com/Printer-Thermistor-Sensor-ReprapComgrow/dp/B0714MR5BC (accessed on 30 March 2021).

51. E-Projects 100EP51210K0 10k Ohm Resistors, 1/2 W, 5\% (Pack of 100). Available online: $\quad$ https://www.amazon.com/Projects-100EP51210K0-10k-ResistorsPack/dp/B0185FIOTA (accessed on 30 March 2021).

52. Arduino Nano. Available online: https://store.arduino.cc/usa/arduino-nano (accessed on 30 March 2021).

53. ELEGOO Nano Board CH340/ATmega328P Without USB Cable, Compatible with Arduino Nano V3.0 (Nano x 3 Without Cable). Available online: https://www.amazon.com/ELEGOO-Arduino-ATmega328P-WithoutCompatible/dp/B0713XK923 (accessed on 30 March 2021). 
54. Monoprice 3-Feet USB A to Mini-B 5pin 28/28AWG Cable (103896) Black. Available online: https://www.amazon.com/Monoprice-3-Feet-mini-B-28AWG103896/dp/B003L18SHC/ (accessed on 30 March 2021).

55. Geekcreit 40pcs FR-4 2.54mm Double Side Prototype PCB Printed Circuit Board. Available online: https:/www.banggood.com/Geekcreit-40pcs-FR-4-2 54mmDouble-Side-Prototype-PCB-Printed-Circuit-Board-p-995732.html (accessed on 30 March 2021).

56. DEPEPE 30 Pcs 40 Pin 2.54mm Male and Female Pin Headers for Arduino Prototype Shield. Available online: https:/www.amazon.com/DEPEPE-2-54mmHeaders-Arduino-Prototype/dp/B074HVBTZ4/ (accessed on 30 March 2021).

57. Plusivo 22AWG Hook up Wire Kit - 600V Tinned Stranded Silicone Wire of 6 Different Colors $\mathrm{x} \quad 23 \mathrm{Ft}$ Each. Available online: https://www.plusivo.com/home/67-plusivo-22awg-hook-up-wire-kit-600v-tinnedstranded-silicone-wire-of-6-different-colors-x-23-ft-each.html (accessed on 30 March 2021).

58. Reflectix Inc. Installation Instructions for Reflectix, Inc. Double Reflective Insulation. Available online: https://images.homedepotstatic.com/catalog/pdfImages/5a/5ab3af4c-631f-416d-af7c-b7aeb41f51a2.pdf (accessed on 30 March 2021).

59. FreeCAD. Available online: https://www.freecadweb.org/ (accessed on $30 \mathrm{March}$ 2021).

60. OpenSCAD. Available online: http://openscad.org (accessed on 30 March 2021).

61. Kirshner, D. Thread-drawing modules for OpenSCAD. Available online: https://dkprojects.net/openscad-threads/ (accessed on 30 March 2021).

62. GNU General Public License Version 3. Available online: https://www.gnu.org/licenses/gpl-3.0.en.html (accessed on 30 March 2021).

63. Corona688. OpenSCAD NPT / Tsmthread 0.4. Available online: https://www.thingiverse.com/thing:3391213 (accessed on 30 March 2021).

64. Creative Commons - Attribution-NonCommercial 3.0 Unported - CC BY-NC 3.0. Available online: https://creativecommons.org/licenses/by-nc/3.0/ (accessed on 30 March 2021).

65. Anzalone, G.; Wijnen, B.; Pearce, J. Multi-Material Additive and Subtractive Prosumer Digital Fabrication with a Free and Open-Source Convertible Delta RepRap 3-D Printer. Rapid Prototyping Journal 2015, 21, 506-519, doi:10.1108/RPJ-09-2014-0113.

66. Ultimaker. Ultimaker Cura 4.7.1. Available online: https://github.com/Ultimaker/Cura (accessed on 30 March 2021)

67. Buckeye Hydro. Should I Use Teflon Tape? Available online: https://www.buckeyehydro.com/blog/should-i-use-teflon-tape/

68. SciPy Optimization and Root Finding (Scipy.Optimize). Available online: https://docs.scipy.org/doc/scipy/reference/optimize.html (accessed on 30 March 2021).

69. Hrisko, J. Arduino Thermistor Theory, Calibration, and Experiment. Maker Portal 2019.

70. Salimov, Y. NTC_Thermistor. Available online: https://github.com/YuriiSalimov/NTC_Thermistor (accessed on 30 March 2021). 
71. Novatec. Hygroscopic VS Non-Hygroscopic Resins. Available online: https://www.ptonline.com/knowledgecenter/plastics-drying/resintypes/hygroscopic-vs-non-hygroscopic-resins (accessed on 8 June 2020).

72. Lee, J.H.; Lim, K.; Hahm, W.; Kim, S. Properties of Recycled and Virgin Poly(Ethylene Terephthalate) Blend Fibers. Journal of Applied Polymer Science 2013, 128, doi:10.1002/app.38502.

73. Zander, N.; Gillan, M.; Burckhard, Z.; Gardea, F. Recycled Polypropylene Blends as Novel 3D Printing Materials. Additive Manufacturing 2018, 25, doi:10.1016/j.addma.2018.11.009.

74. Idrees, M.; Jeelani, S.; Rangari, V. 3D Printed Sustainable Biochar-Recycled PET Composite. ACS Sustainable Chemistry \& Engineering 2018, 6, doi:10.1021/acssuschemeng.8b02283.

75. Baechler, C.; DeVuono, M.; Pearce, J.M. Distributed recycling of waste polymer into RepRap feedstock. Rapid Prototyp. J. 2013, 19, 118-125, doi:10.1108/13552541311302978.

76. Woern, A.L.; McCaslin, J.R.; Pringle, A.M.; Pearce, J.M. RepRapable Recyclebot: Open source 3-D printable extruder for converting plastic to 3-D printing filament. HardwareX 2018, 4, e00026, doi:10.1016/j.ohx.2018.e00026.

77. Karegoudar, T.B.; Pujar, B.G. Degradation of Terephthalic Acid by a Bacillus Species. FEMS Microbiology Letters 1985, 30, 217-220, doi:10.1111/j.15746968.1985.tb01015.x.

78. Vamsee-Krishna, C.; Mohan, Y.; Phale, P. Biodegradation of Phthalate Isomers by Pseudomonas Aeruginosa PP4, Pseudomonas Sp. PPD and Acinetobacter Lwoffii ISP4. Applied microbiology and biotechnology 2006, 72, 1263-9, doi:10.1007/s00253-006-0413-7.

79. Ritala, A.; Häkkinen, S.T.; Toivari, M.; Wiebe, M.G. Single Cell Protein-Stateof-the-Art, Industrial Landscape and Patents 2001-2016. Front Microbiol 2017, 8, doi:10.3389/fmicb.2017.02009.

80. García Martínez, J.B.; Egbejimba, J.; Throup, J.; Matassa, S.; Pearce, J.; Denkenberger, D. Potential of Microbial Protein from Hydrogen for Preventing Mass Starvation in Catastrophic Scenarios. Sustainable Production and Consumption 2020, 25, doi:10.1016/j.spc.2020.08.011.

81. García Martínez, J.B.; Pearce, J.M.; Cates, J.; Denkenberger, D. Methane Single Cell Protein: securing protein supply during global food catastrophes. https://osf.io/94mkg

82. Rosewill. Countertop Portable Electric Food Fruit Dehydrator Machine with Adjustable Thermostat, BPA-Free, 5-Tray, RHFD-15001. Available online: /ip/Rosewill-Countertop-Portable-Electric-Food-Fruit-Dehydrator-Machine-withAdjustable-Thermostat-BPA-Free-5-Tray-RHFD-15001/51409615 (accessed on 31 March 2021).

83. P3. Kill A Watt Meter - Electricity Usage Monitor. Available online: http://www.p3international.com/products/p4400.html (accessed on 31 March 2021).1.

84. $\mathrm{MH}$ Build Series PLA Filament - $1.75 \mathrm{~mm} \quad(1 \mathrm{~kg})$. Available online: https://www.matterhackers.com/store/3d-printer-filament/175mm-pla-filamentred-1-kg (accessed on 31 March 2021).

85. Pearce, J.M. Economic Savings for Scientific Free and Open Source Technology: A Review. HardwareX 2020, 8, e00139, doi:10.1016/j.ohx.2020.e00139. 
86. YamatoADP. Series 220V Vacuum Drying Ovens - Ovens and Furnaces, Vacuum Ovens. Available online: https://www.fishersci.com/shop/products/yamato-adpseries-220v-vacuum-drying-ovens-2/1326320 (accessed on 31 March 2021).

87. Oberloier, S.; Pearce, J. General Design Procedure for Free and Open-Source Hardware for Scientific Equipment. Designs 2017, 2, 2, doi:10.3390/designs2010002.

88. Gibb, A. Building open source hardware: DIY manufacturing for hackers and makers. Pearson Education, 2014.

89. Chagas, A.M. Haves and Have Nots Must Find a Better Way: The Case for Open Scientific Hardware. PLOS Biology 2018, 16, e3000014, doi:10.1371/journal.pbio.3000014.

90. Powell, A. Democratizing Production through Open Source Knowledge: From Open Software to Open Hardware. Media, Culture \& Society 2012, 34, 691-708, doi: $10.1177 / 0163443712449497$.

91. Vacuum Science World. The Fundamentals of Vacuum Science. Available online: https://www.vacuumscienceworld.com/vacuum-science (accessed on 29 March 2021).

92. ABLAZE 3 Gallon Stainless Steel Vacuum Degassing Chamber and 3 CFM Single Stage Pump Kit. Available online: https://www.amazon.com/ABLAZE-GallonStainless-Degassing-Chamber/dp/B076KNYCZ6/ (accessed on 29 March 2021).

93. VAC AERO. International Gas Ballasting of Mechanical Oil Sealed Rotary Vacuum Pumps. Available online: https://vacaero.com/informationresources/vacuum-pump-technology-education-and-training/666-gas-ballastingof-mechanical-oil-sealed-rotary-vacuum-pumps.html (accessed on 30 July 2020).

94. Schach, A. How to Select the Right Vacuum Pump. Labconco 2016. 


\section{Future Work}

The works presented in this thesis shed light on potential areas of further research. Areas of study directly within the field of the instruments in question are discussed in their respective Chapters. Here, areas of study outside the immediate scope of the instruments are explored.

The applications of the mass balance framework are very broad. The digital scale could be adapted to track loads in other systems to aid in performance or status monitoring. 3-D printers could include a load cell to track the consumption of plastic filament or pellets. This would allow printers to pause in the event of filament runout or extruder jams, remembering where in the print the failure occurred. After clearing the jam or adding more filament, the printer could intelligently resume its print, saving potentially large volumes of plastic from being lost to a failed print. Devices which aim to apply a known amount of force during a process could similarly use this sensor in a feedback control system to manage actuator effort. This would be applicable in compression- and tensionbased testing machines, such as tensile testers and melt-flow-indexing machines or 3-D printer extruder drivers. These modifications could be accompanied by organizational changes to the balance firmware to expose internal functionality and separate the serial interface from the balance code library, but no actual changes in behavior would need to be added. Extending the framework even further, it is recognized that the amplifier used for measurements in fact measures a Wheatstone bridge, and that Wheatstone bridges are general sensing circuits with application outside of measuring force. The software/hardware framework could be extended to support different types of sensors, which can be used to measure position, torsion, temperature, and more. The effort required to extend the software library would be small, leaving the majority of work to identifying, assembling, and validating different types of sensors.

The vacuum oven similarly exposed several areas of interest to future research. Airejectors, and more generally converging-diverging nozzles, or even air-flow restricting devices in general, could be examined for use in many areas of research. The plumbing components used on the oven indicate that 3-D printed parts can handle high-pressure airflow, and SLA-type printing can produce parts to very tight tolerances. There is potential to replace the air ejector with a 3-D printed component capable of producing similar levels of vacuums. With that accomplished, it would be fairly straightforward to move on to printing air-flow measurement devices such as venturi nozzles and orifice plates, which must also be manufactured to very tight tolerances, but on a larger scale than the air ejector. New methods for holding a vacuum could also be explored. Developing an open source method for constructing a vacuum chamber has the potential of simplifying the introduction of sensors and power to the inside of the vacuum chamber. Different methods for sealing materials to prevent airflow and off-gassing could be explored, and strength and geometry of vacuum chambers could be optimized by taking advantage of growing technologies in distributed metal additive manufacturing. 


\section{A Copyright documentation}

Chapter 2 is reproduced from "Hubbard, B.R. and Pearce, J.M., 2020. Open-Source Digitally Replicable Lab-Grade Scales. Instruments, 4(3), p.18." This is an open access article distributed by MDPI under the Creative Commons Attribution License which permits unrestricted use, distribution, and reproduction in any medium, provided the original work is properly cited.

Chapter 3 will be published open access as "Open Source Vacuum Oven for LowTemperature Drying” by Benjamin R. Hubbard, Lindsay Putman, Stephen Techtmann, and Joshua M. Pearce. 